\title{
On Turbulent
}

\section{Rayleigh-Bénard Convection in a Two-Phase Binary Gas Mixture}

\author{
Dissertation \\ zur Erlangung des mathematisch-naturwissenschaftlichen Doktorgrades \\ "Doctor rerum naturalium" \\ der Georg-August-Universität Göttingen \\ im Promotionsprogramm \\ Göttingen Graduate School for Neurosciences, \\ Biophysics, and Molecular Biosciences (GGNB) \\ der Georg-August University School of Science (GAUSS)
}

vorgelegt von

Florian Winkel

aus Koblenz

Göttingen 2015 
Mitglieder des Betreuungsausschusses:

\author{
Referent: Dr. Holger Nobach
}

Abt. Hydrodynamik, Strukturbildung und Biokomplexität,

Max-Planck-Institut für Dynamik und Selbstorganisation

Korreferent: Prof. Dr. Andreas Tilgner

Institut für Geophysik, Georg-August-Universität Göttingen

Prof. Dr. Björn Hof

Abt. Nichtlineare Dynamik und Turbulenzen,

Institute of Science and Technology Austria

Tag der mündlichen Prüfung: 27. Oktober 2014 


\begin{abstract}
In this thesis an attempt is made to generate cloud patterns in a laboratory scale experiment. A two-phase binary gas mixture is employed as a physical model system. The fluid mixture is composed of a condensable gas which forms a liquid and a vapor phase and a noncondensable gas which serves as a background or carrier gas. The fluid mixture is confined between a bottom and a top plate. If the fluid mixture is exposed to a constant temperature difference, two intriguing phenomena can be observed.

First a film condensation sets in at the cold top plate that results in the formation of a very regular hexagonal droplet pattern. The temporal evolution of the droplet pattern is quantified and it is shown that a stable mass flux is essential in order to the maintain the hexagonal symmetry of the droplet patten. Second cloud-like patterns occur in a thin layer above the liquid-vapor interface. The dynamics of the cloud-like patterns reveal the turbulent flow inside the gaseous phase. An area-perimeter analysis of the cloud-like patterns results in a fractal dimension that is similar to the one obtained by the fractal analysis of two-dimensional cloud and rain areas in satellite and radar data.

This thesis is meant as a proof of concept which is why most of the results are still qualitative. However, a physical model system is presented that is appropriate in order to study the dynamics of cloud-like patterns in a turbulent Rayleigh-Bénard convection experiment. The origin of the cloud-like patterns is still a matter of debate. Therefore further experiments that could reveal the nature of these patterns must be performed.
\end{abstract}




\section{Contents}

Abstract. .................................... iii

$\begin{array}{lll}1 & \text { Introduction } & 1\end{array}$

1.1 Motivation . . . . . . . . . . . . . . . . . . . . . 1

1.2 Thesis structure $\ldots \ldots \ldots \ldots \ldots$

\begin{tabular}{|ll|l}
2 & Theory & 5
\end{tabular}

2.1 One-component systems $\ldots \ldots \ldots \ldots \ldots$

2.1 .1 Phase diagram of an one-component system . . . . . . . 6

2.2 The liquid-vapor critical point in an one-component system . . . . . 8

$2.2 .1 \quad$ Saturation ratio in an one-component system . . . . . . . . . 9

2.3 Two-component systems $\ldots \ldots \ldots \ldots$

$2.3 .1 \quad$ Phase diagram of a two-component system . . . . . . . . . 10

2.3.2 Thermodynamic state of a two-component system . . . . . . . 16

$2.3 .3 \quad$ Phase coexistence in a two-component system $\ldots \ldots \ldots \ldots$

2.3.4 Thermodynamic equilibrium in a two-component system . . . 19

2.3.5 The liquid-vapor critical point in a two-component system . . . 20

2.3 .6 Saturation ratio in a two-component system . . . . . . . . 20

2.4 Nucleation . . . . . . . . . . . . . . . . . . . . . . . . . . . 21

2.5 Hydrodynamic instabilities . . . . . . . . . . . . . . . 25 
2.5 .1 Rayleigh-Bénard instability . . . . . . . . . . . . . . 25

2.5 .2 Rayleigh-Taylor instability . . . . . . . . . . . . . . . . 30

3 Experimental Setup 33

3.1 Experimental apparatus $\ldots \ldots \ldots \ldots$

3.2 Optical setup . . . . . . . . . . . . . . . . . . . . 37

3.3 Calibration procedure $\ldots \ldots \ldots \ldots \ldots \ldots$

3.3 .1 Temperature calibration $\ldots \ldots \ldots \ldots \ldots$

3.3 .2 Pressure calibration. . . . . . . . . . . . . . . . . 40

3.3 .3 Plate alignment . . . . . . . . . . . . . . . . . . 42

3.3 .4 Temperature correction $\ldots \ldots \ldots \ldots \ldots$. . . . . . 43

3.3 .5 Scale calibration . . . . . . . . . . . . . . . . . . . . 44

3.4 Filling procedure for $\mathrm{SF}_{6}$ and $\mathrm{He} \ldots \ldots \ldots \ldots$

$3.4 .1 \quad$ Filling procedure for $\mathrm{SF}_{6} \ldots \ldots \ldots \ldots \ldots \ldots$

3.4 .2 Filling procedure for $\mathrm{He} \ldots \ldots \ldots \ldots$. . . . . . . 50

3.4 .3 Temperature scenarios for the main pressure vessel . . . . . . . 51

$\begin{array}{lll}4 & \text { Results } & 53\end{array}$

4.1 General remarks and observations . . . . . . . . . . . . . . 53

4.1 .1 Thermal diffusion . . . . . . . . . . . . . . . . . . . . . 54

4.1 .2 Mass diffusion . . . . . . . . . . . . . . . . 57

4.2 Droplet Formation $\ldots \ldots \ldots \ldots \ldots$

4.2 .1 Image analysis $\ldots \ldots \ldots \ldots \ldots$. . . . . . . . . . 64

4.2 .2 Heater switched off . . . . . . . . . . . . . . . . 68

4.2 .3 Heater switched on . . . . . . . . . . . . . . . 74

4.2 .4 Discussion . . . . . . . . . . . . . . . . . . . . . . . 79

4.3 Cloud Patterns . . . . . . . . . . . . . . . . . . . . . . . . . . . 82 
$4.3 .1 \quad$ Cloud dynamics $\ldots \ldots \ldots \ldots \ldots$. . . . . . . . . . 84

$4.3 .2 \quad$ Cloud pattern analysis $\ldots \ldots \ldots \ldots$. . . . . . . . . 90

4.3 .3 Discussion . . . . . . . . . . . . . . . . . . . 96

$\begin{array}{lll}5 \text { Conclusion } & 108\end{array}$

5.1 Summary . . . . . . . . . . . . . . . . . . . . . . . 108

5.2 Outlook . . . . . . . . . . . . . . . . . . . . . 110

\begin{tabular}{ll}
\hline $\mathrm{A}$ & 112
\end{tabular}

A.1 Determination of the molar concentration of $\mathrm{He}$ in the gaseous phase . 112

A.2 Estimation of the Rayleigh number for the liquid phase of $\mathrm{SF}_{6}$. . . . 115

A.3 Estimation of the Rayleigh number for the gaseous phase of $\mathrm{SF}_{6}$ and $\mathrm{He} 16$

A.4 Turbulent plume velocity and turbulent mixing time. . . . . . . . . 118

Acknowledgements . . . . . . . . . . . . . . . . . . . . . . . . . . 121

\begin{tabular}{ll}
\hline Bibliography & 122
\end{tabular} 


\section{Chapter 1}

\section{Introduction}

\subsection{Motivation}

Most systems in nature are located far away from equilibrium. If a system is driven out of equilibrium, it experiences a restoring force that drives the system back into its equilibrium position. An example for a system which is always out of equilibrium is the earth's atmosphere. The incoming solar radiation heats the atmosphere and the surface of the earth. Local temperature gradients result in a local variation of the density which in turn causes updrafts and downdrafts of warm and cold air respectively. Pressure gradients build up which are balanced by the appearance of strong advective and convective flows. The advective and convective flows are important for the circulation of heat and mass in the atmosphere. The solar radiation also heats the surface of the oceans. The water at the surface starts to evaporate and the water vapor is transported to the atmosphere by parcels of warm air. The temperature of the lower troposphere decreases with increasing height. The decrease in temperature causes the water vapor to condense which results in the formation of clouds which in turn generate precipitation. In reality all these processes are experienced as weather. 
The underlying physical processes which determine the climate and the weather are quite complex and a subject of current research. For instance, the impact of clouds on the climate is not yet fully understood. Clouds are important because they absorb and reflect part of the sun's and the earth's radiation and therefore have a tremendous effect on the local climate where they occur. Laboratory experiments, field studies as well as theoretical models can help to gain a better understanding of the physics of clouds. A detailed description of the processes which are involved in the formation of clouds is beyond the scope of this thesis. What should be pointed out is that the mechanisms behind the formation of clouds comprise processes such as heat and mass transfer as well as the transition between different thermodynamic phases. The aforementioned processes also play an important role in the experiments which are performed in this thesis. In order to study the formation and the dynamics of clouds in a laboratory environment, new experimental methods and model systems must be developed which allow a simplified description of the complex environmental conditions. In physical experiments this is achieved by reducing the degrees of freedom of the system which is under investigation. However, despite a strong simplification of the reality, the experiments should still be meaningful enough to provide a reasonable explanation for potential observations [41, 59].

The mechanism how clouds are formed in the atmosphere is called heterogeneous nucleation. A heterogeneous nucleation occurs if the air is saturated with water vapor. The water vapor in the atmosphere condenses on the surface of aerosol or dust particles which serve as nucleation sites for the water molecules. If no nucleation sites are present, then water droplets can only be formed by a mechanism which is called homogeneous nucleation. A homogeneous nucleation is likely to occur if the air is greatly supersaturated with water vapor. However, supersaturated conditions hardly occur in nature and therefore must be realized in laboratory exper- 
iments. Various experimental techniques exist in order to create a supersaturation under laboratory conditions. For instance, by employing a thermal diffusion cloud chamber (TDCC), a region of supersaturation is created in a binary gas mixture by applying a temperature difference between the bottom and the top plate. If the supersaturation exceeds a critical value, then the probability rises that a large number of droplets are nucleated at the same time. However, in a TDCC the thermal convection is suppressed and heat and mass are transported by diffusion. The question arose, whether or not a region of supersaturation could also be realized in a turbulent convection experiment by employing a two-phase binary gas mixture and if so, whether this supersaturation is large enough so that a large number of droplets can be nucleated. The idea is that the nucleated droplets accumulate to form cloud-like patterns and that these patterns might exhibit similar characteristics which are typical for clouds in the atmosphere. In the results section of this thesis it is shown that cloud-like patterns are observed in a turbulent Rayleigh-Bénard convection experiment by employing a two-phase binary gas mixture as a physical model system. However, it is not entirely clarified why the cloud patterns do occur. Therefore, further experiments must be carried out that could help to answer the question whether the cloud patterns are formed by nucleated droplets or are caused by other effects like critical opalescence or a change in the refractive index of the fluid mixture.

\subsection{Thesis structure}

The thesis is structured as follows. Chapter 2 gives a short introduction into the theory of phase transitions and phase equilibria. The chapter includes a short summary of the classical theory of nucleation. Two types of hydrodynamic instabilities are briefly discussed that are crucial in order to understand the results in Chapter 4 In 
Chapter 3 a short description of the experimental apparatus and the optical setup is given. The calibration procedure for the thermistors and the pressure gauge is described as well. The results of the experiments are presented in Chapter 4 . In Section 4.1 general aspects of the experimental system are discussed. Section 4.2 describes the droplet pattern that forms on the underside of the top plate. The cloud-like patterns are presented in Section 4.3. A discussion of the observed phenomena is given at the end of each section. A short conclusion and an outlook for future experiments are presented in Chapter 5 . 


\section{Chapter 2}

\section{Theory}

Since a two-phase binary gas mixture is employed as a physical model system, this chapter starts with a short description of phase diagrams, phase changes and crit-

ical phenomena. The theory about one-component and two-component systems is mainly a summary of selected chapters in [53] and in [68] that are relevant for this thesis. In the subsequent section a short introduction to the theory of classical nucleation is given. Finally the Rayleigh-Bénard and the Rayleigh-Taylor instability are presented as a paradigm for a buoyancy-driven and an interfacial instability respectively.

\subsection{One-component systems}

A homogeneous system forms a thermodynamic phase if it has specific properties that are uniform throughout its entire dimension [53]. A fluid or one-component system can be constituted in a solid, in a liquid or in a gaseous state. A system is called heterogeneous if two or more phases are in coexistence with each other at the same time [53]. A pure or one-component fluid such as sulfur hexafluoride may either 
be located in a homogeneous state as a pure gaseous phase or in a heterogeneous state if a liquid and a vapor phase coexist. The pressure $p$ and the temperature $T$ are normally used to represent the different phases of a fluid in a $p-T$ diagram which is called a phase diagram.

The experiments presented in this thesis are conducted in a two-phase binary gas mixture that is composed of sulfur hexafluoride $\left(\mathrm{SF}_{6}\right)$ and helium $(\mathrm{He})$. In the temperature range under consideration, $\mathrm{SF}_{6}$ is the condensable component and forms a liquid and a vapor phase. He is the noncondensable component which remains in the gaseous phase. The component which forms a liquid and a vapor phase is called the solvent and is less volatile. The component that remains in the gaseous phase is called the solute and is the more volatile component. In the subsequent paragraph the phase diagram of an one-component and a two-component system is briefly explained and discussed.

\subsubsection{Phase diagram of an one-component system}

Fig. 2.1 shows the schematic drawing of a $p-T$ phase diagram of an one-component fluid. The solid red line marks the liquid-vapor coexistence line (LVCL) that separates the liquid and the vapor phase. For a certain temperature $T$, the coexistence of two phases is only possible if the pressure $p$ adjusts according to the LVCL. The coexistence line ends in the critical point $(\mathrm{CP})$ that is defined by the critical pressure $p_{\mathrm{c}}$ and the critical temperature $T_{\mathrm{c}}$. Above the critical point the liquid and the vapor phase become indistinguishable and the fluid changes into a supercritical state. The vertical and the horizontal black dashed line in Fig. 2.1 illustrate the scenario of an isothermal compression and an isobaric heating of the fluid respectively. The two scenarios are briefly discussed. Therefore it is assumed that an one-component fluid is confined in a closed container that is terminated at one side by a movable pis- 


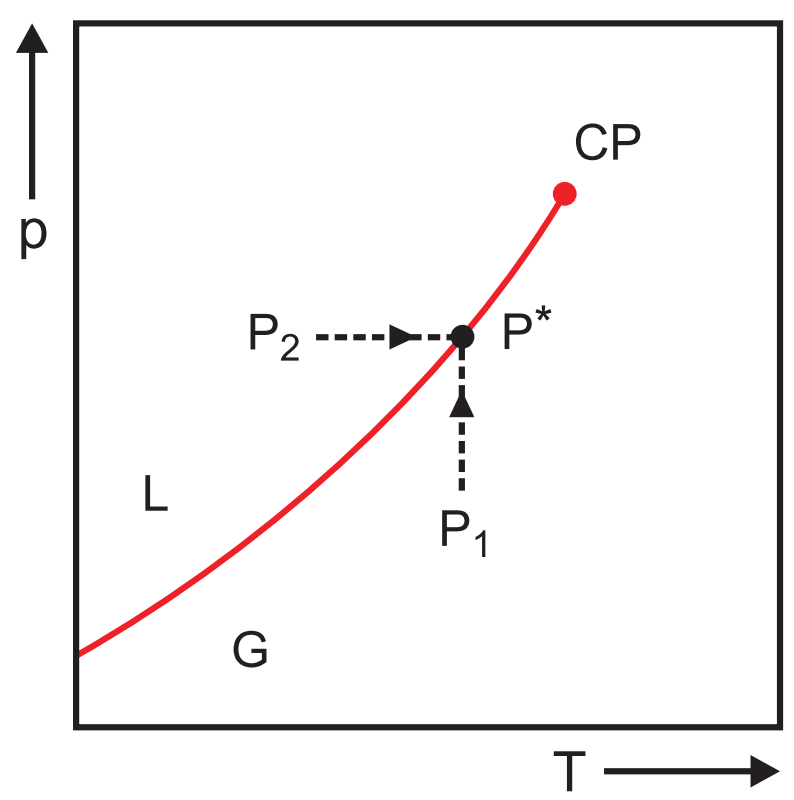

Figure 2.1: Schematic drawing of the phase diagram of an one-component fluid. $\mathrm{CP}$ = critical point, $\mathrm{G}=$ gaseous phase, $\mathrm{L}=$ liquid phase.

ton. The first scenario $\left(\mathrm{P}_{1}-\mathrm{P}^{*}\right)$ describes the isothermal compression and the second scenario $\left(\mathrm{P}_{2}-\mathrm{P}^{*}\right)$ the isobaric heating of the system:

- Isothermal compression $\left(\mathrm{P}_{1}-\mathrm{P}^{*}\right)$ :

The fluid is located in the gaseous phase $\left(\mathrm{P}_{1}\right)$ and is kept at a constant temperature. If the volume of the container is reduced by moving the piston, the pressure increases until the LVCL is encountered. At the coexistence line a condensation sets in and a liquid phase starts to form. If the volume is further reduced, the pressure does not change unless all the gas is converted into a liquid. In the end $\left(\mathrm{P}^{*}\right)$ a single liquid phase remains in the container.

- Isobaric heating $\left(\mathrm{P}_{2}-\mathrm{P}^{*}\right)$ :

The fluid is located in the liquid phase $\left(\mathrm{P}_{2}\right)$ and the pressure inside the container is kept constant. If the temperature of the fluid is increased, the volume of the container expands because the density of the liquid decreases with temperature. At the LVCL vapor bubbles start to appear and a vapor phase begins to form. If 
the system is further heated, the volume of the container increases and the temperature does not change unless all the liquid is converted into a gas. If a single gaseous phase exists in the container $\left(\mathrm{P}^{*}\right)$ and the system is further heated, the gaseous phase continues to expand.

Fig. 2.2 shows the $p-T$ phase diagram of pure $\mathrm{SF}_{6}$ as an example for the phase diagram of an one-component fluid. The solid green line represents the LVCL. Above the coexistence line the system forms a pure liquid phase while it remains in a pure gaseous phase at pressures below the coexistence line. Whenever a liquid and a vapor phase coexist, the system is represented by a point on the LVCL. The closed red circle at the upper end of the LVCL marks the critical point. At the critical point, the density of the liquid equals the density of the vapor phase and the fluid becomes supercritical with the critical density $\rho_{\mathrm{c}}$.

\subsection{The liquid-vapor critical point in an one-component system}

If an one-component system moves along the LVCL towards higher temperatures and higher pressures, then the density of the liquid phase decreases and the density of the vapor phase increases. The critical point with $p_{\mathrm{c}}$ and $T_{\mathrm{c}}$ marks the terminal point in Fig. 2.2 where the densities of the liquid and the vapor phase become equal. By applying the Clausius-Clapeyron equation, the differences in the molar entropy $s$ and the molar volume $v$ of a liquid (L) and a vapor phase $(\mathrm{G})$ in coexistence are defined as

$$
\left(s_{\mathrm{G}}-s_{\mathrm{L}}\right)=A\left(v_{\mathrm{G}}-v_{\mathrm{L}}\right)
$$




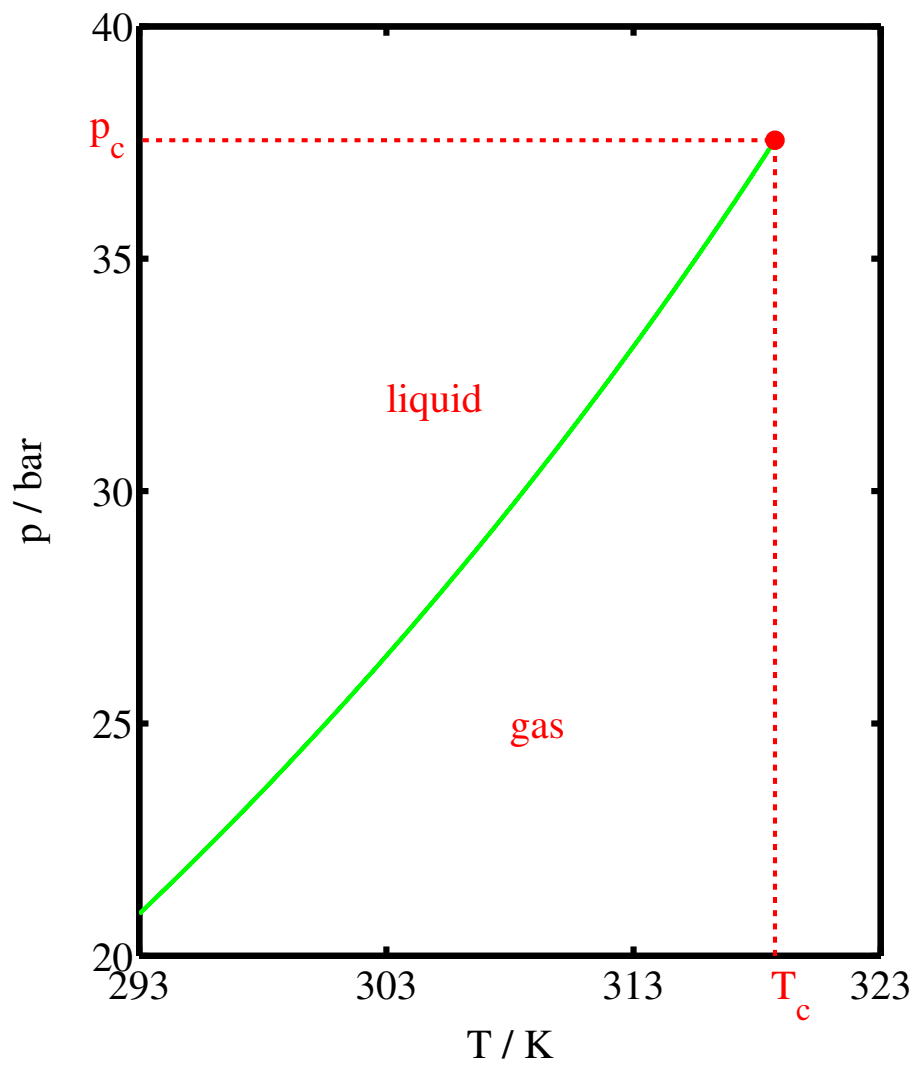

Figure 2.2: $p-T$ phase diagram of pure $\mathrm{SF}_{6}$.

where $A$ is a constant that is derived at the critical point according to $A=(\partial p / \partial T)_{v}$. Eq. (2.1) shows that the molar volumes $v_{\mathrm{G}}$ and $v_{\mathrm{L}}$ and the molar entropies $s_{\mathrm{G}}$ and $s_{\mathrm{L}}$ become identical at the critical point and as a consequence the latent heat of vaporization vanishes [53].

\subsubsection{Saturation ratio in an one-component system}

Suppose a liquid and a vapor phase coexist in a heterogeneous system. For liquidvapor phase transitions, the saturation ratio $S$ defines how far away the vapor phase of the system is located from an equilibrium state [68]. The saturation ratio is defined as

$$
S=\frac{p_{\mathrm{v}}(T)}{p_{\mathrm{vs}}(T)},
$$


where $p_{\mathrm{v}}$ is the partial vapor pressure and $p_{\mathrm{vs}}$ is the saturation vapor pressure of an one-component fluid for a specific temperature [68]. If more molecules are in the vapor phase than normally would be in the equilibrium state, then the vapor is supersaturated and $S>1$. If a liquid phase exists and $S<1$, then the liquid begins to evaporate until the liquid phase has vanished or until the vapor phase is saturated and $S=1$. If $S>1$, then the vapor starts to condense if a liquid phase is present or a surface where the vapor can condense on.

\subsection{Two-component systems}

\subsubsection{Phase diagram of a two-component system}

In general it can be distinguished between homogeneous and heterogeneous mixtures. A homogeneous mixture consists of a single thermodynamic phase while a heterogeneous mixture comprises different thermodynamic phases. Gases are normally fully miscible and that is why gas mixtures are always homogeneous. If one of the components in the gas mixture exists in two thermodynamic phases that means in a liquid and a vapor phase, then the mixture is heterogeneous [53]. This section discusses the phase diagram of a two-component system where one of the components forms a liquid and a vapor phase.

Fig. 2.3 shows the schematic drawing of a $p-T$ phase diagram of a two-component fluid. The subscripts 1 and 2 denote the two constituents of the fluid mixture. The phase diagram illustrates the case if component 1 is a condensable gas and forms a liquid and a vapor phase while component 2 is a noncondensable gas and remains in the gaseous phase. The solid red line marks the critical line (CL) that separates the two-phase from the one-phase region. The lower half of the critical line is called the dew point line (DL) and separates the one-phase region where both constituents 


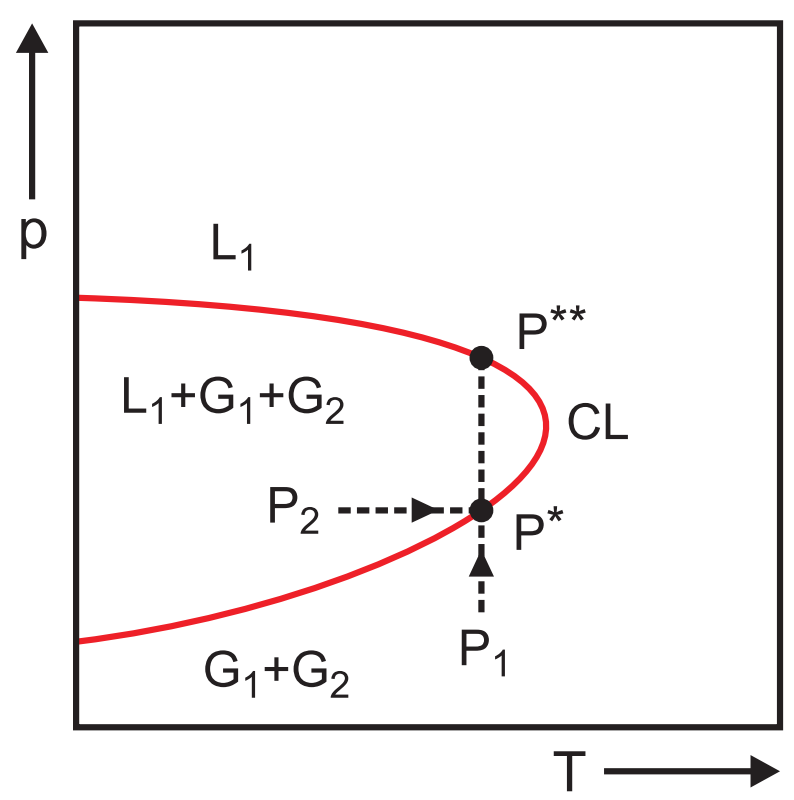

Figure 2.3: Schematic drawing of the phase diagram of a two-component fluid. CL $=$ critical line, $G_{1}=$ gaseous phase of component $1, G_{2}=$ gaseous phase of component $2, \mathrm{~L}_{1}=$ liquid phase of component 1 .

exist in a gaseous phase from the two-phase region where the liquid phase is formed by component 1 and the gaseous phase is formed by a mixture of the components 1 and 2. The upper half of the critical line is called the bubble point line (BL) and separates the one-phase region where the component 1 forms a sole liquid phase from the two-phase region. The vertical and the horizontal black dashed line in Fig. 2.3 illustrate the case of an isothermal compression and an isobaric heating respectively. The two scenarios are briefly discussed. Therefore it is assumed that the two-component fluid is confined in a closed container that is terminated at one side by a movable piston. The first scenario $\left(\mathrm{P}_{1}-\mathrm{P}^{*}-\mathrm{P}^{* *}\right)$ describes the isothermal compression and the second scenario $\left(\mathrm{P}_{2}-\mathrm{P}^{*}\right)$ the isobaric heating of the system:

- Isothermal compression $\left(\mathrm{P}_{1}-\mathrm{P}^{*}-\mathrm{P}^{* *}\right)$ :

The fluid is located in the one-phase region $\left(\mathrm{P}_{1}\right)$ and the temperature of the container is kept constant. Both constituents form a gaseous phase $G_{1}$ and $G_{2}$. If the 
volume of the container is reduced by moving the piston, the pressure increases until the point $\mathrm{P}^{*}$ on the $\mathrm{CL}$ is reached. At the critical line the component 1 starts to condense and forms a liquid phase $\mathrm{L}_{1}$. The gaseous phase is composed of $\mathrm{G}_{1}$ and $G_{2}$ and the system is located in the two-phase region. If the volume is further reduced, then the vapor phase of the component $G_{1}$ is converted into a liquid while the fraction of the component $\mathrm{G}_{2}$ in the gaseous phase is increasing. If the volume is further reduced, then the component $G_{2}$ is solved in the liquid phase $\mathrm{L}_{1}$. Since the component 2 is the more volatile component, the pressure in the system must be increased in order to solve $\mathrm{G}_{2}$ completely in $\mathrm{L}_{1}$. If the entire vapor phase $G_{1}$ is converted into a liquid and the component $G_{2}$ is completely diluted in the liquid phase $\mathrm{L}_{1}$, then the system is located at the point $\mathrm{P}^{* *}$ on the $\mathrm{CL}$ and a sole liquid phase $\mathrm{L}_{1}$ exists in the container. The system is located in the one-phase region above the bubble point line.

- Isobaric heating $\left(\mathrm{P}_{2}-\mathrm{P}^{*}\right)$ :

The fluid is located in the two-phase region $\left(\mathrm{P}_{2}\right)$ and the pressure inside the container is kept constant. If the temperature of the fluid mixture is increased, then the volume of the container expands because the liquid phase $\mathrm{L}_{1}$ begins to evaporate and the mole fraction of $G_{1}$ in the gaseous phase increases. The component $\mathrm{G}_{2}$ that is diluted in the liquid phase $\mathrm{L}_{1}$ is also released. If the fluid mixture is further heated, then more and more liquid is evaporating unless the entire liquid phase $L_{1}$ is converted into a gas. The system is now located at the point $\mathrm{P}^{*}$ on the $\mathrm{CL}$. If the temperature is further increased, then the system moves into the onephase region where a gaseous phase composed of $G_{1}$ and $G_{2}$ exists. If the system is heated even more, then the gaseous phase continues to expand.

As mentioned in Section 1.1, the motivation for this thesis was whether or not a supersaturation can be realized in a turbulent Rayleigh-Bénard convection experiment. 
A two-phase binary gas mixture composed of $\mathrm{SF}_{6}$ as a condensable and $\mathrm{He}$ as a noncondensable gas is employed as a physical model system. The phase diagram of a $\mathrm{SF}_{6}$ and He mixture can either be determined experimentally or it can be derived from other known phase diagrams. Since the measurement of the phase diagram for a mixture of $\mathrm{SF}_{6}$ and He causes an enormous effort, the $p-T$ phase diagram is qualitatively derived from the $p-T$ phase diagram of a mixture of Xe and He. The critical properties of $\mathrm{Xe}$ and $\mathrm{SF}_{6}$ are similar as can be seen in Table 2.1. Whenever two substances have similar critical properties, their corresponding phase diagrams are also of the same type. The $p-T$ phase diagram of a mixture of $\mathrm{Xe}$ and $\mathrm{He}$

\begin{tabular}{lccc}
\hline Parameter & $\mathrm{SF}_{6}$ & $\mathrm{He}$ & $\mathrm{Xe}$ \\
\hline${ }^{\mathrm{a}} T_{\mathrm{c}} / \mathrm{K}$ & 318.72 & 5.19 & 289.74 \\
${ }^{\mathrm{a}} p_{\mathrm{c}} / \mathrm{bar}$ & 37.60 & 2.27 & 58.40 \\
${ }^{\mathrm{a}} V_{\mathrm{c}} / \mathrm{cm}^{3} \mathrm{~mol}^{-1}$ & 198.40 & 57.30 & 118.00 \\
${ }^{\mathrm{a}} M_{\mathrm{w}} / \mathrm{g} \mathrm{mol}^{-1}$ & 146.056 & 4.003 & 131.290 \\
\hline
\end{tabular}

a Data taken from [57].

Table 2.1: Critical constants of the mixture components. $T_{\mathrm{C}}$ denotes the critical temperature, $p_{\mathrm{c}}$ the critical pressure, $V_{\mathrm{c}}$ the critical molar volume and $M_{\mathrm{W}}$ the molecular weight.

is shown in Fig. 2.4a and was reproduced from [19]. In addition a qualitative version of the $p-T$ phase diagram of a mixture of $\mathrm{SF}_{6}$ and He was computed with a commercial software licensed from [52]. The result is shown in Fig. 2.4b for three different concentrations. Both phase diagrams look similar as expected. The solid and the dashed line in Fig. $2.4 \mathrm{~b}$ separate the two-phase from the one-phase region. The upper solid and the lower dashed line mark the bubble point and the dew point line respectively. For a larger concentration of $\mathrm{He}$, the two-phase region is extended and the critical line shifts to the right. The black dotted lines mark the temperature 


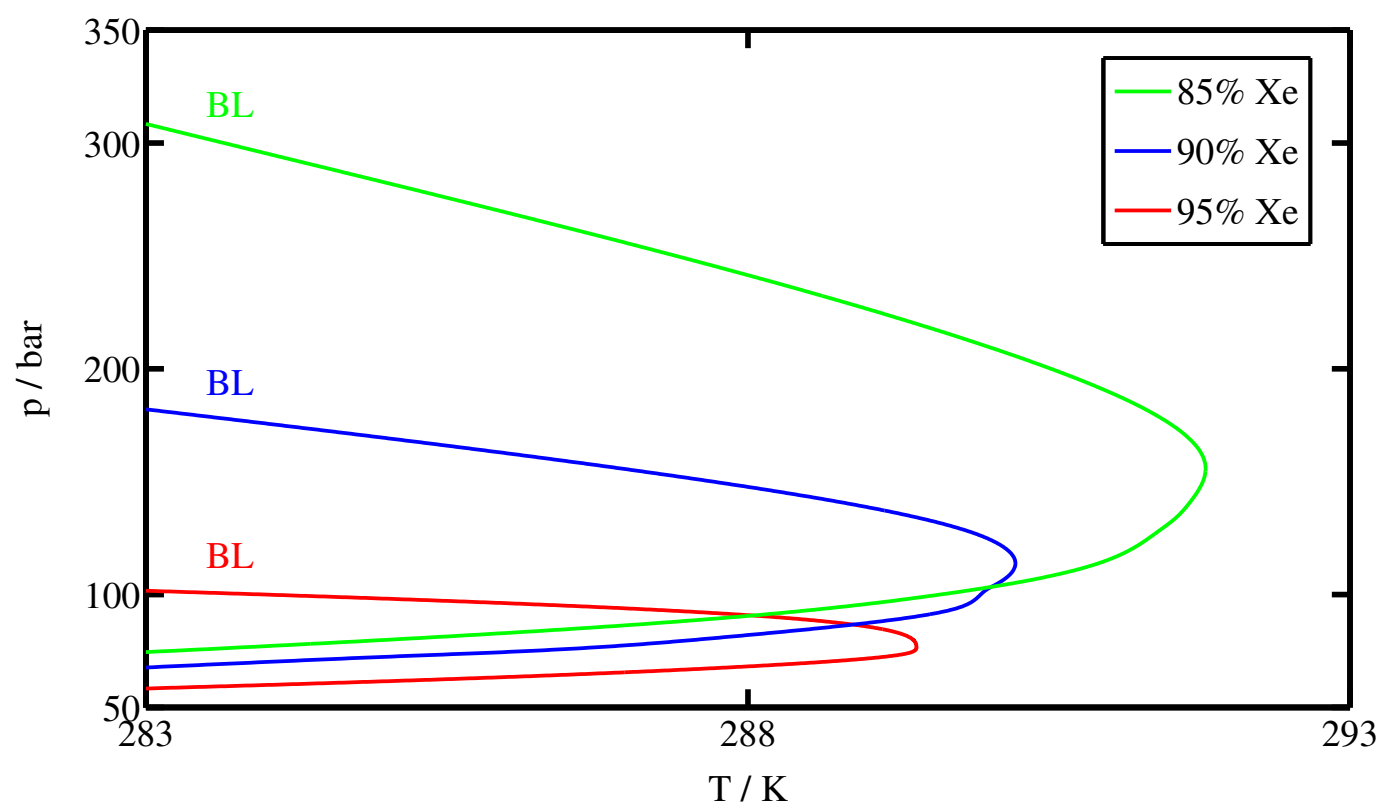

(a)

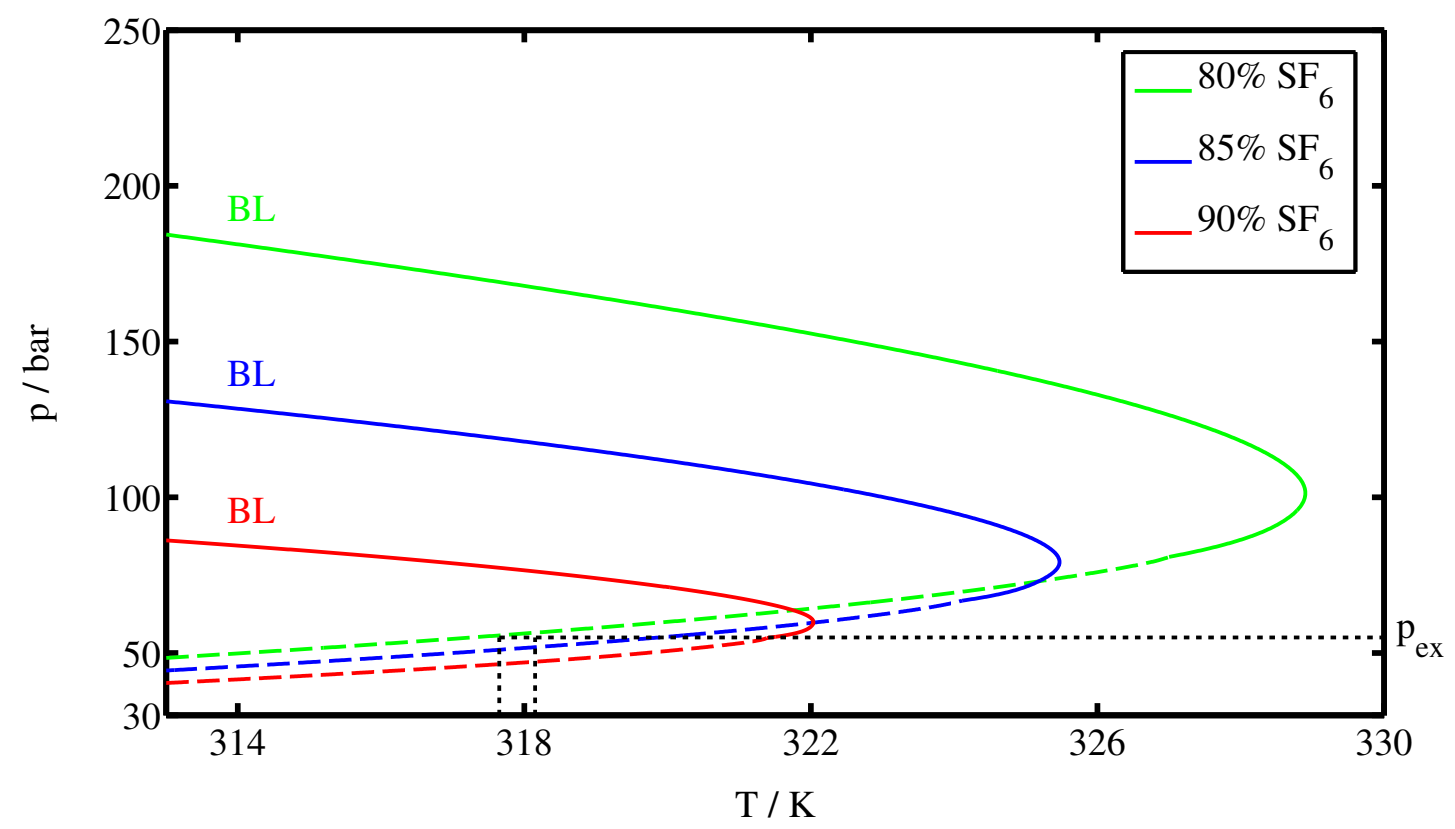

(b)

Figure 2.4: (a) and (b) show the $p-T$ phase diagram of a mixture of Xe and He and of a mixture of $\mathrm{SF}_{6}$ and $\mathrm{He}$ for three different concentrations respectively. 


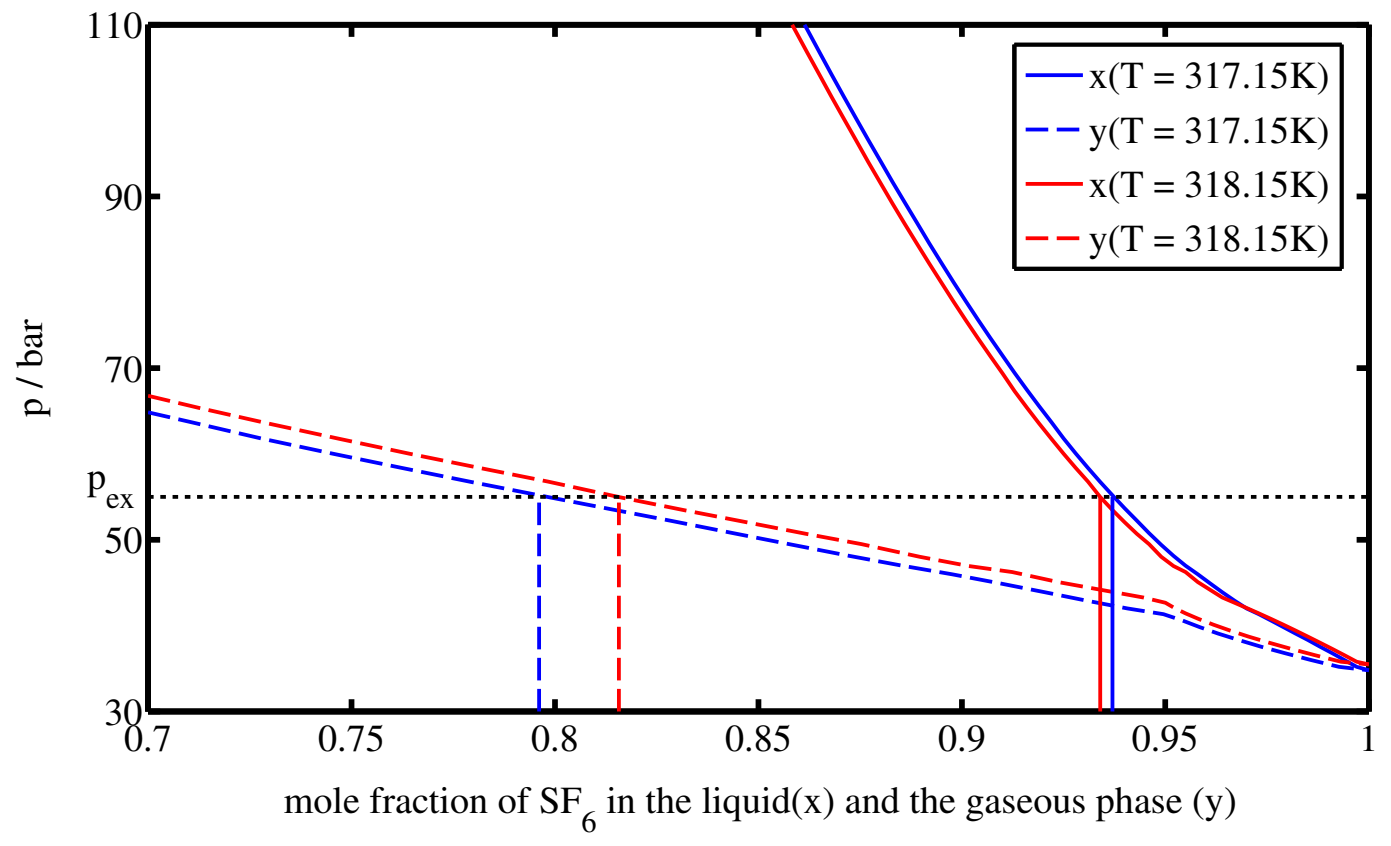

Figure 2.5: $p-x-y$ phase diagram of a mixture of $\mathrm{SF}_{6}$ and He. $x$ and $y$ denote the mole fraction of $\mathrm{SF}_{6}$ in the liquid and the gaseous phase respectively.

range and the pressure at which the experiments in this thesis were conducted. The results in Chapter 4 were obtained with a molar concentration of $\mathrm{He}$ in the gaseous phase of approximately $14.5 \%$. The black dotted lines in Fig. $2.4 \mathrm{~b}$ show that the system is located in the two-phase region which is evident since a liquid and a gaseous phase did always coexist during the experiments. If $\mathrm{SF}_{6}$ forms a liquid and a vapor phase, then the concentration of $\mathrm{He}$ in the liquid phase is usually different from the concentration in the gaseous phase. Fig. 2.5 illustrates the $p-x-y$ phase diagram of a mixture of $\mathrm{SF}_{6}$ and $\mathrm{He}$ for two different temperatures that are labeled in blue and red. The solid and the dashed lines mark the concentration of $\mathrm{SF}_{6}$ in the liquid and the gaseous phase respectively. The concentration of He in the gaseous phase is usually larger compared to the concentration in the liquid phase of $\mathrm{SF}_{6}$. For instance, at $T=317.15 \mathrm{~K}$, the mole fraction of $\mathrm{He}$ in the gaseous phase is roughly $20 \%$ while in the liquid phase of $\mathrm{SF}_{6}$ it is only about $6 \%$. The mole fraction of He diluted in the 
liquid phase of $\mathrm{SF}_{6}$ is much smaller since gases are only slightly soluble in liquids [58]. The concentration of $\mathrm{He}$ in the gaseous phase depends also on the temperature. With decreasing temperature the solubility of $\mathrm{He}$ in the liquid phase of $\mathrm{SF}_{6}$ decreases and the concentration in the gaseous phase increases. This seems logical since the vapor pressure of $\mathrm{SF}_{6}$ increases with increasing temperature and as a consequence the concentration of $\mathrm{He}$ in the gaseous phase decreases.

\subsubsection{Thermodynamic state of a two-component system}

In terms of the internal energy, the thermodynamic state of a system with two components 1 and 2 is defined by the extensive variables of the entropy $S$, the volume $V$ and the number of moles of the two components $N_{i},(i=1,2)$. The differential form of the internal energy $U\left(S, V, N_{i}\right)$ is known to be

$$
\mathrm{d} U=T \mathrm{~d} S-p \mathrm{~d} V+\mu_{1} \mathrm{~d} N_{1}+\mu_{2} \mathrm{~d} N_{2},
$$

where $T$ denotes the temperature, $p$ the pressure and $\mu_{i},(i=1,2)$ the chemical potential of the two components [53]. Another representation of the thermodynamic state of the fluid is given by the differential form of the Gibbs free energy $G\left(T, p, N_{i}\right)$ that is defined as

$$
\mathrm{d} G=-S \mathrm{~d} T+V \mathrm{~d} p+\mu_{1} \mathrm{~d} N_{1}+\mu_{2} \mathrm{~d} N_{2} .
$$

Suppose $G$ is an extensive function of the variables $N_{i},(i=1,2)$, then the Gibbs free energy can be written as

$$
G=\mu_{1} N_{1}+\mu_{2} N_{2} .
$$


By combining Eqs. (2.4) and (2.5), the Gibbs-Duhem relation

$$
-S \mathrm{~d} T+V \mathrm{~d} p-N_{1} \mathrm{~d} \mu_{1}-N_{2} \mathrm{~d} \mu_{2} \geq 0
$$

can be derived. The Gibbs-Duhem criterion means that a thermodynamically stable phase of the system is given by a minimum of the Gibbs free energy. The Gibbs free energy in Eq. (2.4) can also be expressed by molar quantities, in this case $g$ is defined as $g=G / N$ and the mole fraction of the components 1 and 2 are defined as $x_{1}=N_{1} / N$ and $x_{2}=N_{2} / N$ respectively. The total number of moles is $N=N_{1}+N_{2}$. By applying the aforementioned definitions, Eq. (2.5) can be written as

$$
g=\mu_{1} x_{1}+\mu_{2} x_{2}
$$

and the molar Gibbs free energy is given by

$$
\mathrm{d} g=-s \mathrm{~d} T+v \mathrm{~d} p+\mu_{1} \mathrm{~d} x_{1}+\mu_{2} \mathrm{~d} x_{2} .
$$

In ideal mixture as shown in [53], the molar representation of the Gibbs free energy for two components is defined as

$$
g=x g_{2}+(1-x) g_{1}+x R T \ln x+(1-x) R T \ln (1-x) .
$$

In Eq. (2.9) $g_{1}$ and $g_{2}$ denote the molar Gibbs free energies for the two components 1 and 2 and $x$ the mole fraction of the component 2 under the assumption that $x \ll 1$. For a regular mixture the expression in Eq. (2.9) changes to

$$
g=g^{\mathrm{E}}+x g_{2}+(1-x) g_{1}+x R T \ln x+(1-x) R T \ln (1-x),
$$


where $g^{\mathrm{E}}(T, p, x)$ denotes the molar excess Gibbs free energy. $g^{\mathrm{E}}$ describes the deviation of the behavior of a real mixture from an ideal mixture. If a binary mixture can be described by $g^{\mathrm{E}}$, then the mixture is called a regular mixture [53]. The excess Gibbs free energy is of the form

$$
g^{\mathrm{E}}=w x(1-x)
$$

where $w$ is a function of $p$ and T. $g^{\mathrm{E}}$ is constructed in a way that $g^{\mathrm{E}} \rightarrow 0$ if $x \rightarrow 0$ or $x \rightarrow 1$ that means if the concentration of the component 2 becomes zero or one. If $g^{\mathrm{E}} \rightarrow 0$, then the expression in Eq. (2.10) reduces to the expression of the Gibbs free energy for an ideal mixture in Eq. (2.9).

\subsubsection{Phase coexistence in a two-component system}

In a two-component system where a liquid phase (L) and a gaseous phase (G) coexist, the fraction of the number of moles in the liquid and the gaseous phase are given by $r_{\mathrm{L}}=N_{\mathrm{L}} / N$ and $r_{\mathrm{G}}=N_{\mathrm{G}} / N$ respectively, where $N=N_{\mathrm{L}}+N_{\mathrm{G}}$ is the total number of moles. If $x_{i},(i=1,2)$ and $y_{i},(i=1,2)$ denote the mole fraction of the two components in the liquid and the gaseous phase respectively, then the number of moles of component 2 in the liquid and the gaseous phase is $x_{2} N_{\mathrm{L}}$ and $y_{2} N_{\mathrm{G}}$ respectively. The total number of moles of component 2 in the mixture is $N_{2}=x_{2} N_{\mathrm{L}}+y_{2} N_{\mathrm{G}}$. Since the mole fraction of component 2 in the mixture is $x=N_{2} / N$, the expression

$$
x=x_{2} r_{\mathrm{L}}+y_{2} r_{\mathrm{G}}
$$

can be derived which is called the lever rule. The lever rule allows to determine the composition of the liquid and the gaseous phase from a $T-x$ or $p-x$ phase 
diagram. With $r_{\mathrm{L}}+r_{\mathrm{G}}=1$, the following relations

$$
r_{\mathrm{L}}=\frac{x-y_{2}}{x_{2}-y_{2}}
$$

and

$$
r_{\mathrm{G}}=\frac{x_{2}-x}{x_{2}-y_{2}}
$$

can be derived for the component 2. Because the temperature and the pressure in the system are constant, $x_{2}$ and $y_{2}$ remain unchanged and the same is true for the molar Gibbs free energy of the two phases $g_{\mathrm{L}}^{*}=g\left(T, p, x_{2}\right)$ and $g_{\mathrm{G}}^{*}=g\left(T, p, y_{2}\right)$. Thus the molar Gibbs free energy can be written as

$$
g=r_{\mathrm{L}} g_{\mathrm{L}}^{*}+r_{\mathrm{G}} g_{\mathrm{G}}^{*} .
$$

The chemical potential $\mu=\partial g / \partial x$ is constant in the range $x_{2}<x<y_{2}$ and the following condition

$$
g_{\mathrm{L}}^{*}-\mu^{*} x_{2}=g_{\mathrm{G}}^{*}-\mu^{*} y_{2}
$$

for the coexistence of the two phases can be derived [53].

\subsubsection{Thermodynamic equilibrium in a two-component system}

If a two-component mixture is in a thermodynamic equilibrium, then the temperature $T$, the pressure $p$ and the chemical potential $\mu_{1}$ and $\mu_{2}$ of the component 1 and 2 respectively must be equal everywhere in the system. That means in equilibrium the following conditions must be fulfilled

$$
T_{\mathrm{L}}=T_{\mathrm{G}}, \quad p_{\mathrm{L}}=p_{\mathrm{G}}, \quad \mu_{1 \mathrm{~L}}=\mu_{1 \mathrm{G}}, \quad \mu_{2 \mathrm{~L}}=\mu_{2 \mathrm{G}} .
$$


By applying Eq. (2.15), it can be shown that

$$
g_{\mathrm{L}}\left(T, p, x_{2}\right)-\mu^{*} x_{2}=g_{\mathrm{G}}\left(T, p, y_{2}\right)-\mu^{*} y_{2}
$$

with $g_{\mathrm{L}}^{*}=g_{\mathrm{L}}\left(T, p, x_{2}\right)$ and $g_{\mathrm{G}}^{*}=g_{\mathrm{G}}\left(T, p, y_{2}\right)$ [53]. Furthermore it can be shown that Eq. (2.18) is equivalent to the expression

$$
\mu_{1 \mathrm{~L}}\left(T, p, x_{1}\right)=\mu_{1 \mathrm{G}}\left(T, p, y_{1}\right) \text { and } \mu_{2 \mathrm{~L}}\left(T, p, x_{2}\right)=\mu_{2 \mathrm{G}}\left(T, p, y_{2}\right) \text {. }
$$

The expressions in Eqs. (2.18) and (2.19) are the conditions for the thermodynamic equilibrium in a two-component mixture and can be used to determine $x_{2}$ and $y_{2}$ for the component 2. More details can be found in [53].

\subsubsection{The liquid-vapor critical point in a two-component system}

The two-phase region in Fig. 2.3 is bounded by the critical line and each point on the critical line can be considered as a single critical point. For the case of a twocomponent fluid, the critical line represents an infinite set of critical points. However, for the two-component system in Fig. 2.3, the definition of the critical point in Section 2.2 is not applicable since a terminal point like the one in Fig. 2.1 does not exist anymore. That means the two-component system cannot exist in a state where the critical temperature and the critical pressure occur simultaneously.

\subsubsection{Saturation ratio in a two-component system}

In [68] the saturation vapor pressure of a mixture component $i$ is defined as

$$
p_{i, \mathrm{v}}\left(x_{i}, T\right)=\Gamma_{i}\left(x_{i}, T\right) x_{i} p_{i, \mathrm{vs}}(T)
$$


Here $x_{i}$ denotes the mole fraction of the component $i$ in the liquid phase, $\Gamma_{i}\left(x_{i}, T\right)$ the activity coefficient and $p_{i, v s}(T)$ the saturation vapor pressure of the component $i$. For an ideal mixture $\Gamma_{i}=1$ while for a regular mixture $\Gamma_{i} \neq 1$. According to [68], the liquid phase activity $A_{i, \mathrm{~L}}$ is defined as

$$
A_{i, \mathrm{~L}} \equiv \frac{p_{i, \mathrm{v}}\left(x_{i}, T\right)}{p_{i, \mathrm{vs}}(T)}=\Gamma_{i} x_{i}
$$

For a two-component system, the saturation ratio $S_{i}$ indicates how saturated the gaseous phase of component $i$ is with respect to the liquid phase of the mixture

$$
S_{i} \equiv \frac{p_{i, \mathrm{G}}}{p_{i, \mathrm{~V}}\left(x_{i}, T\right)}=\frac{p_{i, \mathrm{G}}}{p_{i, \mathrm{vs}}(T) A_{i, \mathrm{~L}}} .
$$

Furthermore the gas phase activity $A_{i, \mathrm{G}}$ is defined as

$$
A_{i, \mathrm{G}}=\frac{p_{i, \mathrm{G}}}{p_{i, \mathrm{vs}}(T)}
$$

$A_{i, \mathrm{G}}$ indicates how saturated the gaseous phase of component $i$ is with respect to the pure liquid phase of the component $i$. The saturation ratio and the gas phase activity are related as follows

$$
S_{i}=\frac{A_{i, \mathrm{G}}}{A_{i, \mathrm{~L}}} .
$$

For an one-component system the gas phase activity and the saturation ratio are identical $A_{\mathrm{G}}=S$ and the liquid phase activity becomes $A_{\mathrm{L}}=1$ [68].

\subsection{Nucleation}

If a new phase starts to form in a homogeneous system, the process how the new phase is formed is called nucleation. A new phase can be nucleated either by a 
heterogeneous nucleation or by a homogeneous nucleation. Heterogeneous nucleation describes the process when the nucleation of a new phase starts on the surface of nucleation sites such as aerosol particles or on solid surfaces like a window or a wall [35, 68]. A heterogeneous nucleation occurs in the atmosphere when saturated water vapor condenses on the surface of dust or salt particles and causes the formation of clouds or fog.

Homogeneous nucleation describes the formation of a new phase that occurs in the absence of any nucleation sites or other surfaces where the molecules can condense on. Suppose a homogeneous and one-component system is located in a gaseous state. In order that a new phase can be formed by a homogeneous nucleation, one condition is that a supersaturation must occur in the system. If the degree of supersaturation is sufficiently large, then density fluctuations in the vapor phase can initiate the formation of short-lived molecule clusters [21]. If the supersaturation is only weak, then the clusters dissociate again. If the supersaturation further increases, then the probability rises that a molecule cluster persists over time and is capable of growing. Furthermore, if the supersaturation exceeds a critical value, then abruptly a large number of stable clusters arise that act as nucleation sites for other molecules. In the beginning the clusters consist of only a few molecules. The growth of the clusters continues by condensing more and more molecules that are also called monomers. The growth of the clusters continues until macroscopic droplets are formed. The latent heat released by the condensation is transferred to the vapor phase and results in a temperature increase which reduces the degree of supersaturation in the vapor phase [21]. A homogeneous nucleation occurs also at the critical point of an one-component fluid. If the system changes from a supercritical to a subcritical state, then density fluctuations induce the formation of a new phase that is nucleated by a homogeneous nucleation. The density fluctuations at 


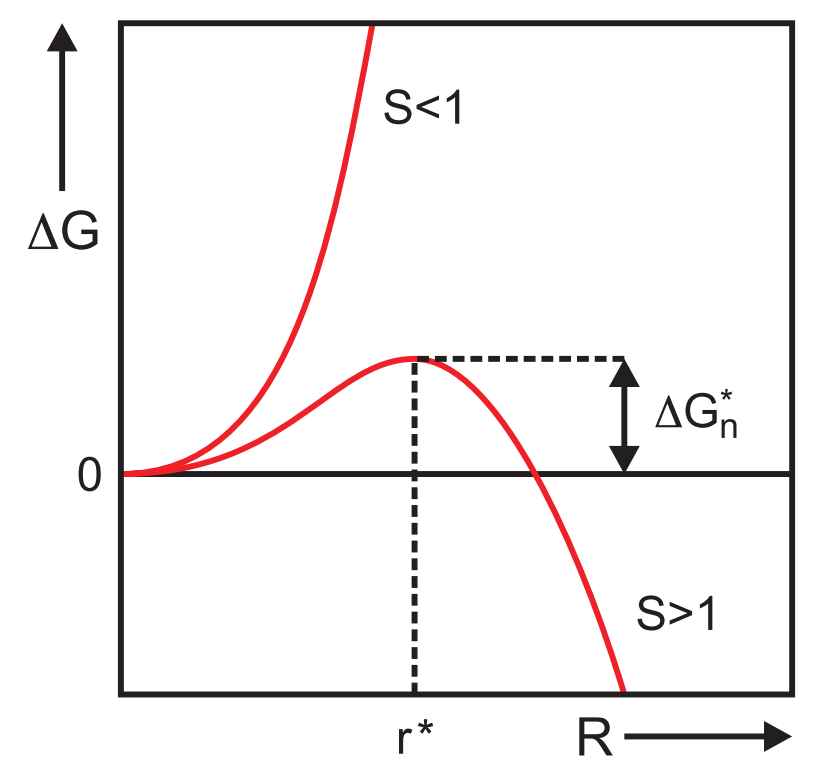

Figure 2.6: Schematic drawing of the free energy barrier for a homogeneous nucleation from a supersaturated vapor phase.

the critical point cause a phenomenon which is called critical opalescence [17, 67].

In the following a brief introduction to the classical theory of nucleation is given that is mainly adopted from [2]. One way how a supersaturated atmosphere can be created in an experimental environment is by an isentropic expansion or by an isobaric cooling of a pure vapor phase. In the following the thermodynamic requirements will be explained that are essential for the formation of a stable droplet in a supersaturated environment. The change in the Gibbs free energy $\Delta G$ for a cluster of $n$ molecules in a closed system is given by

$$
\Delta G=n\left(\mu_{\mathrm{L}}-\mu_{\mathrm{V}}\right)+4 \pi r^{2} \sigma,
$$

where $r$ denotes the radius, $\sigma$ the surface tension of the cluster and $\mu_{\mathrm{L}}$ and $\mu_{\mathrm{V}}$ the chemical potential of the molecules in the liquid and the vapor phase respectively. By assuming isothermal conditions, the Gibbs-Duhem equation can be used to replace 
the first term on the right side of Eq. (2.25) with

$$
\mathrm{d}\left(\mu_{\mathrm{L}}-\mu_{\mathrm{V}}\right)=n\left(v_{\mathrm{L}}-v_{\mathrm{V}}\right) \mathrm{d} p_{\mathrm{V}}
$$

where $v_{\mathrm{L}}$ and $v_{\mathrm{V}}$ denote the specific volume of the molecules in the liquid and the vapor phase respectively. Under the assumption that the vapor behaves like an ideal gas, Eq. (2.26) can be transformed into

$$
\mu_{\mathrm{L}}-\mu_{\mathrm{V}}=-k_{\mathrm{B}} T \ln S
$$

Here $k_{\mathrm{B}}$ denotes the Boltzmann constant and $S$ the degree of supersaturation of the vapor phase. By combining Eq. (2.25) and Eq. (2.27), the Gibbs free energy can be written as

$$
\Delta G=4 \pi r^{2} \sigma-\frac{4}{3} \pi r^{3} \frac{k_{\mathrm{B}} T \ln S}{v_{\mathrm{L}}} .
$$

The first term on the right side of Eq. (2.28) accounts for the work that is necessary to extend the liquid surface against the surrounding vapor phase if a molecule joins the cluster. The second term on the right side of Eq. (2.28) considers the change in the free Gibbs energy of the system if a molecule is removed from the vapor phase and added to the cluster. Fig. 2.6 illustrates Eq. (2.28) for two different saturation values. For the case of $S<1$, the energy needed for the growth of a cluster is increasing with increasing radius. It is unlikely that the cluster grows in size and therefore a nucleation in a subsaturated environment is not possible. For the case of $S>1$, a critical radius $r^{*}$ exists at which the Gibbs free energy barrier for the formation of a stable cluster reaches its maximum. For values of $r<r^{*}$, the energy $\Delta G$ is first increasing with increasing radius and reaches a maximum at $r=r^{*}$. For values of $r>r^{*}$, the energy barrier $\Delta G$ starts to decrease due to the contribution of the volume 
term that starts to overcome the surface tension term. The critical radius $r^{*}$ deter-

mines whether a new phase is stable or not. Clusters smaller than $r^{*}$ are on average dissociating while clusters larger than $r^{*}$ persist and grow to a macroscopic size in the bulk of the vapor phase. The derivation of $r^{*}=\mathrm{d}(\Delta G) / \mathrm{d} r$ is given by

$$
r^{*}=\frac{2 \sigma v_{\mathrm{L}}}{k_{\mathrm{B}} T \ln S}
$$

and the Gibbs free energy $\Delta G^{*}=\Delta G\left(r^{*}\right)$ at the position $r^{*}$ is

$$
\Delta G^{*}=-\frac{16}{3} \frac{\pi \sigma^{3} v_{\mathrm{L}}^{2}}{\left(k_{\mathrm{B}} T \ln S\right)^{2}} .
$$

As already mentioned, the degree of supersaturation $S$ is crucial in order that a stable cluster can form. Therefore a homogeneous nucleation is likely to occur in a homogeneous system with a high level of supersaturation and in the absence of any nucleation sites. A more comprehensive overview of the theory of nucleation can be found in [32, 38, 40, 49, 72].

\subsection{Hydrodynamic instabilities}

\subsubsection{Rayleigh-Bénard instability}

Suppose a fluid is confined between two parallel, ideal conducting, infinitely extended and horizontally aligned plates that are separated in a distance $H$ apart from each other. The temperature of the bottom and the top plate is denoted with $T_{\mathrm{b}}$ and $T_{\mathrm{t}}$ respectively. Initially both plates have the same temperature $T_{\mathrm{b}}=T_{\mathrm{t}}=T$. If a small temperature difference $\Delta T=T_{\mathrm{b}}-T_{\mathrm{t}}>0$ is applied between the bottom and the top plate, then heat is transported from the bottom to the top plate via conduc- 
tion and the fluid remains in a steady and motionless state. However, the fluid at the bottom plate at $z=0$ has a larger temperature than the fluid at the top plate at $z=H$. Thus the density of the fluid at $z=0$ is smaller than the density at $z=H$. If the fluid is considered to consist of stratified fluid layers whose density is increasing in the positive $z$-direction, then the density of the fluid layer $i$ is smaller than the density of the fluid layer $\mathrm{i}+1$ which means $\rho_{i}\left(z_{i}\right)<\rho_{i+1}\left(z_{\mathrm{i}+1}\right)$ for $z_{i}<z_{\mathrm{i}+1}$. The fluid layer $i$ tends to rise upwards due to the buoyancy forces acting on the fluid layer. On the other hand the fluid layer $i+1$ experiences a gravitational force and tends to sink downwards. However, if the applied temperature difference is small, the stabilizing dissipative forces due to viscous drag and thermal diffusion overcome the destabilizing forces due to buoyancy and gravity and prevent the fluid layer from becoming unstable. If the temperature difference is further increased, then the density variation becomes larger and at a critical temperature difference $\Delta T_{\mathrm{c}}$, the buoyancy and the gravitational force begin to overcome the dissipative forces. As a consequence, the fluid layers become unstable and warm fluid from below rises upwards while cold fluid from above falls downwards. The heat is now transported by thermal convection and this paradigm is also called Rayleigh-Bénard convection (RBC). The Rayleigh number $(R a)$ is the dimensionless control parameter that characterizes the ratio of the buoyancy to the frictional force [15, 48]. The Rayleigh number is defined as

$$
R a=\frac{\alpha g H^{3} \Delta T}{\kappa v}
$$

where $\alpha$ denotes the thermal expansion coefficient, $g$ the gravitational constant, $H$ the cell height, $v$ the kinematic viscosity and $\kappa$ the thermal diffusivity of the fluid. Another important quantity is the dimensionless Prandtl number $(\mathrm{Pr})$ that is defined 
as

$$
\operatorname{Pr}=\frac{\tau_{\kappa}}{\tau_{v}}=\frac{v}{\kappa}
$$

where $\tau_{\kappa}=H^{2} / \kappa$ denotes the thermal relaxation time and $\tau_{v}=H^{2} / v$ the viscous relaxation time. The Prandtl number indicates which heat dissipation mechanism is dominating in the fluid. For gases $\operatorname{Pr}$ is usually of the order of $\mathcal{O}(1)$.

In reality it is not possible to create ideal boundary conditions in laboratory experiments. In RBC experiments the fluid is confined in a container with a height $H$ and a width $W$. Typically the thermal convection is affected by the boundaries of the enclosure, in particular the sidewalls. Thus it makes sense to define the aspect ratio

$$
\Gamma=\frac{W}{H}
$$

in order to account for the geometry of the system. A large value of $\Gamma$ means that the effect of the sidewalls on the thermal convection in the center of the enclosure can be neglected. The Rayleigh number indicates how far away the system is located from an equilibrium state. By performing a linear stability analysis of the equations of motion, it is shown [15, 25] that a system with two rigid boundaries at the bottom and the top is getting unstable at a critical Rayleigh number of

$$
R a_{\mathrm{c}}=1707.8
$$

whereas a system with a rigid boundary at the bottom and a stress-free boundary at the top is getting unstable at a critical Rayleigh number of

$$
R a_{\mathrm{c}}=1100.7 \text {. }
$$


The onset of the thermal convection in Eqs. (2.34) and (2.35) is independent of the Prandtl number and the material parameters of the fluid. However, for the nonlinear properties of the thermal convection the dependence $R a(P r)$ must be considered.

\section{Convection regimes for low Prandtl number fluids}

In [12] different convection regimes for $R a$ up to $10^{6}$ and $\operatorname{Pr}$ up to $10^{4}$ are presented that were identified in numerical simulations and experimental investigations. Since the Prandtl number for the gaseous phase of $\mathrm{SF}_{6}$ and $\mathrm{He}$ is of the order of $\mathcal{O}(1)$, the distinct convection regimes that can occur in systems with $\operatorname{Pr} \sim 1$ and $R a$ up to $10^{6}$ are briefly mentioned here. In fluids with $\operatorname{Pr} \sim 1$ and $R a<R a_{\mathrm{c}}$, infinitesimal perturbations in the system decay and do not affect the stable fluid layer. In the critical regime at $R a=R a_{\mathrm{c}}$, a steady-state motion sets in with a critical wavenumber $k_{\mathrm{c}}$ as the stable mode of the first convective instability [25]. For $R a>R a_{\mathrm{c}}$ in the supercritical regime, the fluid layer is convectively unstable what results in the formation of steady rolls that persist up to $R a \sim 5 \times 10^{3}$ [25]. If $R a$ is further increased, oscillations occur along the axis of the rolls that mark the transition to a regime with aperiodic time-dependent convection. The transition to turbulent convection occurs around $R a \sim 2 \times 10^{4}$. For even larger $R a$ in the range of $R a \sim 10^{5}-10^{7}$, the turbulent convection exhibits the typical structure of large-scale convection cells [12] that are maintained by the ejection of thermal plumes from the thermal boundary layers.

\section{Thermal plumes}

In turbulent RBC the thermal and the viscous boundary layers develop at the surface of the bottom and the top plate. It is well-known that a temperature drop occurs in the thermal boundary layers and that the temperature throughout the bulk of the fluid remains nearly isothermal [6, 12, 20]. The convection patterns in turbulent RBC 
are characterized by the appearance of a large-scale circulation (LSC) that is extended over the height and the width of the convection cell and by intermittent ejections of thermal plumes from the lower and the upper boundary layer. By varying $R a$ and Pr, four different regimes of plume growth can be identified and classified according to the dimensions of the thermal and the viscous boundary layer [43].

\section{Large-scale circulation}

In turbulent $\mathrm{RBC}$ for $\operatorname{Pr}>0.35$, the rising and descending plumes are mainly responsible for the vertical heat transport. The horizontal dimensions of a thermal plume are comparable to the thickness of the thermal boundary layer where the plume arises [43]. The plumes that are released from the thermal boundary layer at the bottom move laterally toward the sidewall and form upwelling jets in a region near the sidewall of the container. Cold plumes at the top move in the opposite direction and form downward jets at the opposite sidewall of the container. The LSC has a two-dimensional structure and appears for $R a$ up to $10^{13}$ [43]. The structure of the LSC can be classified into three parts: first a thin viscous boundary layer, second a homogeneously mixed core region in the center of the container and third an intermediate buffer region that is dominated by the thermal plumes [43].

In [43] it is described that the transport of the thermal plumes along the bottom and the top boundary layer is theoretically related to the propagation of thermal waves that arise due to temperature oscillations in the thermal boundary layer. In a theoretical model it was proposed that the temperature oscillations and the propagating waves are induced by thermal plumes that impact into the thermal boundary layer from the opposite side of the container and cause an instability of the thermal boundary layer. If the induced waves move toward the sidewall of the container, sheets of warm fluid get ejected from the top of these waves that tend to rise upwards. As the 
sheets move toward the sidewall, they split into columnar structures that form the thermal plumes [43]. Some of the plumes can be released from the boundary layer before the sidewall is reached and move into the central region of the container. However, most of the plumes hit the sidewall where they form a cluster and flow upward as a jet. When the jet impacts on the top plate, waves are produced that move across the top plate and create new plumes that flow downward to the bottom. Thus a self-sustaining large-scale flow is maintained by the alternating warm and cold plumes that are ejected in the lower and the upper thermal boundary layer. The upward and downward flowing jets at the sidewalls of the container result from the clustering of these plumes. The jets circulate the fluid in the container and thus create the large-scale circulation structure [43].

\subsubsection{Rayleigh-Taylor instability}

Suppose a liquid film is suspended on the underside of a horizontally oriented surface. At the bottom the surface of the liquid film is bounded by a subjacent gaseous phase. The interface between the liquid film and the gaseous phase is stabilized by the surface tension of the fluid. Initially the interface is plane and unperturbed. The gravitational force is acting on the interface and destabilizes the liquid film. If the surface of the liquid film becomes unstable due to perturbations, then the interface gets corrugated and periodic crests and sinks begin to appear. If liquid is accumulating in the crests, then droplets start to form. If a droplet reached a critical size, it detaches from the liquid film and falls downwards. The type of interfacial instability is known as the Rayleigh-Taylor instability (RTI). A first analytical description of the problem was given by Rayleigh in 1883 [61]. In 1950 the problem was experimentally investigated by Lewis [44] and Taylor [66]. In the following paragraph two references from the literature are presented that describe distinct models of how a 
liquid film on the underside of a horizontal surface can be stabilized.

In [8] the situation is described when the surface of a liquid film on the underside of a flat horizontal plate is heated from below. Initially the liquid film is in equilibrium with the adjacent gaseous phase. The temperature dependence of the surface tension of the fluid is neglected. Perturbations of the thickness of the liquid film result in the formation of a corrugated surface. Depressions develop in regions where the fluid is warmer and elevations where the fluid is colder. In [8] the evaporation in the depressions is considered as a stabilizing mechanism. The fluid evaporates in the thicker regions while it condenses in the thinner regions. If the destabilizing effect of the gravitational force overcomes the stabilizing mechanism of the evaporation and the condensation, then the liquid film becomes Rayleigh-Taylor unstable. By performing a linear stability analysis, the critical wavelength $\lambda_{c}$ can be determined to

$$
\lambda_{\mathrm{c}}=2 \pi\left[\frac{\sigma}{g\left(\rho_{\mathrm{L}}-\rho_{\mathrm{G}}\right)}\right]^{1 / 2} .
$$

Here $g$ denotes the gravitational constant, $\rho_{\mathrm{L}}$ and $\rho_{\mathrm{G}}$ the density of the liquid film and the gaseous phase respectively and $\sigma$ the surface tension of the liquid film. If $\lambda>\lambda_{\mathrm{c}}$, then the liquid-vapor interface is unstable since the amplitude of the perturbation grows exponentially in time. For wavelengths $0<\lambda<\lambda_{\mathrm{c}}$, the perturbations do decay and the surface tension of the fluid stabilizes the liquid-vapor interface. Another model for the stabilization of the surface of a liquid film is described in [10]. Here the liquid film is subjected to a vertical temperature difference by heating the liquid film from below. It is shown that the Rayleigh-Taylor instability can be suppressed by a vertical temperature gradient. Perturbations of the thickness of the liquid film result in the formation of thicker and thinner regions where the fluid is warmer and colder respectively. By assuming a temperature dependent surface tension, fluid flows along the interface from warmer regions where the surface tension 
is lower toward colder regions where the surface tension is higher. Thus the liquid film is stabilized by the temperature dependent surface tension. If the destabilizing gravitational force overcomes the stabilizing force due to the temperature dependent surface tension, then the system becomes Rayleigh-Taylor unstable at long wavelengths in which the most unstable wavelength is infinite. A more comprehensive overview of interfacial instabilities can be found in [54]. 


\section{Chapter 3}

\section{Experimental Setup}

\subsection{Experimental apparatus}

A schematic drawing of the convection apparatus which is used for the experiments is shown in Fig. 3.1. The apparatus consists of a main pressure vessel that houses the inner can stack and the convection cell. Fig. 3.2 illustrates the squared plexiglas cell in which the convection takes place. The width and the height of the cell is $W=65.1 \mathrm{~mm}$ and $H=21.6 \mathrm{~mm}$ respectively. The thickness of the bottom and the sidewalls of the cell is $S W=1 \mathrm{~mm}$. The convection cell is confined between a bottom and a top plate. Both plates have a diameter of $D_{\mathrm{p}}=101.6 \mathrm{~mm}$ and a height of $H_{\mathrm{p}}=9.5 \mathrm{~mm}$. A polished silicon plate is used as a bottom plate and the top plate is a polished sapphire plate. The aspect ratio of the convection cell is

$$
\Gamma=\frac{W}{H} \approx 3 .
$$

A foil heater is glued to the underside of the bottom plate and allows to heat the bottom plate. The top plate is kept at a constant temperature by an internal water bath. An aquarium pump circulates the water in the internal water bath in the directions 


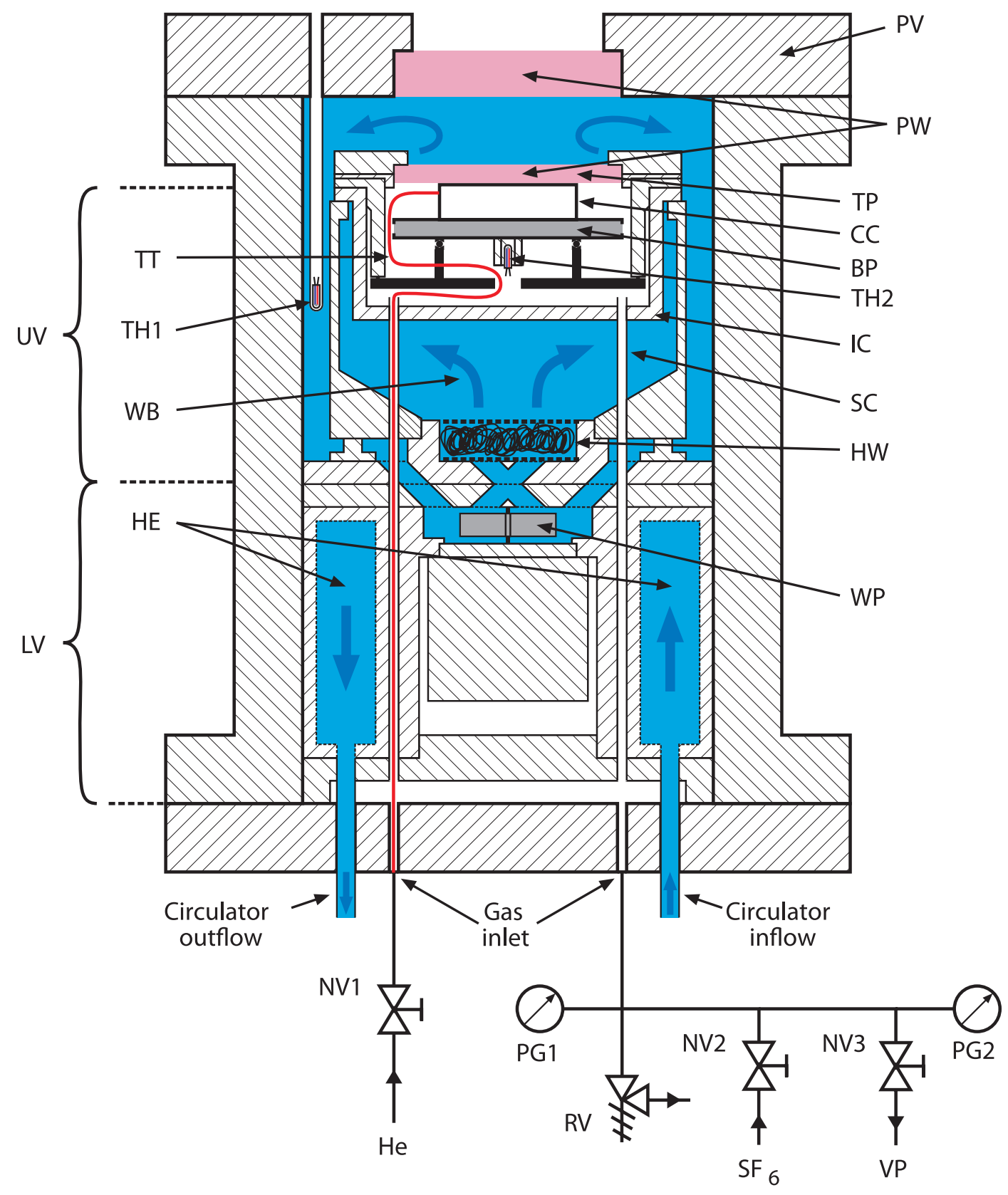

Figure 3.1: Schematic drawing of the experimental apparatus (not to scale). Apparatus: $\mathrm{BP}=$ bottom plate, $\mathrm{CC}=$ convection cell, $\mathrm{SC}=$ steel conduits, $\mathrm{HE}=$ heat exchanger, $\mathrm{HW}=$ heating wire, $\mathrm{IC}=$ inner can, $\mathrm{LV}=$ lower volume, $\mathrm{PV}=$ pressure vessel, $\mathrm{SW}=$ sapphire $\left(\mathrm{Al}_{2} \mathrm{O}_{3}\right)$ window, $\mathrm{TH} 1=$ water bath thermistor, $\mathrm{TH} 2=$ bottom plate thermistor, $\mathrm{TP}=$ top plate, $\mathrm{UV}=$ upper volume, $\mathrm{WB}=$ water bath, $\mathrm{WP}=$ water pump. Outer piping: NV1 = needle valve $1, \mathrm{NV} 2=$ needle valve $2, \mathrm{NV} 3=$ needle valve $3, \mathrm{PG} 1$ = pressure gauge $1, \mathrm{PG} 2=$ pressure gauge $2, \mathrm{RV}=$ pressure relief valve, $\mathrm{VP}=$ vacuum pump. 
pointed out by the dark blue arrows in Fig. 3.1. The internal water bath is heated by a teflon insulated heating wire (AWG32, Alpha Wire Inc.) that supplies a maximum heating power of about $120 \mathrm{~W}$. By using a heat exchanger which is connected to a refrigerated circulator (Neslab RTE 7, Thermo Electron Corporation), the heat of the internal water bath is transported away. The heat exchanger is also used to adjust the internal water bath to a certain working temperature.

The voltage for the bottom plate heater and the heating wire is supplied by two power supplies (Agilent E3634A, Agilent Technologies Inc.). The top steel lid of the main pressure vessel has an opening in the middle that holds a sapphire plate with a diameter of $D_{\mathrm{p}}=101.6 \mathrm{~mm}$ and a height of $H_{\mathrm{p}}=32.6 \mathrm{~mm}$. The sapphire window allows for an optical access to the convection cell from outside the pressure vessel. Two stainless steel conduits are used to connect the inner can to the interior stack that houses the heat exchanger and the water pump. The conduits connect the lower volume of the apparatus with the upper volume as shown in Fig. 3.1. High pressure seals (Conax Technologies) are used to feed the electrical wires for the voltage supply and for the temperature measurement through the bottom lid. The thermistors TH1 and TH2 are used to measure the temperature of the top and the bottom plate respectively. The type of thermistor that is used for the experiments is a $100 \mathrm{k} \Omega$ precision thermistor probe (Fenwal Electronics Inc.) with a negative temperature coefficient (NTC).

The bottom of Fig. 3.1 shows the outer piping of the convection apparatus that is used for the gas supply and for the pressure measurement. The gas inlet is managed by two needle valves NV1 and NV2 that are connected to a He and an $\mathrm{SF}_{6}$ gas bottle respectively. One end of a $1 \mathrm{~mm}$ thick teflon tube (marked in red) is connected to the pipe connection for the He gas inlet and the other end of the teflon tube is tightly connected to the convection cell. Via the teflon tube the He gas is blown directly into the 


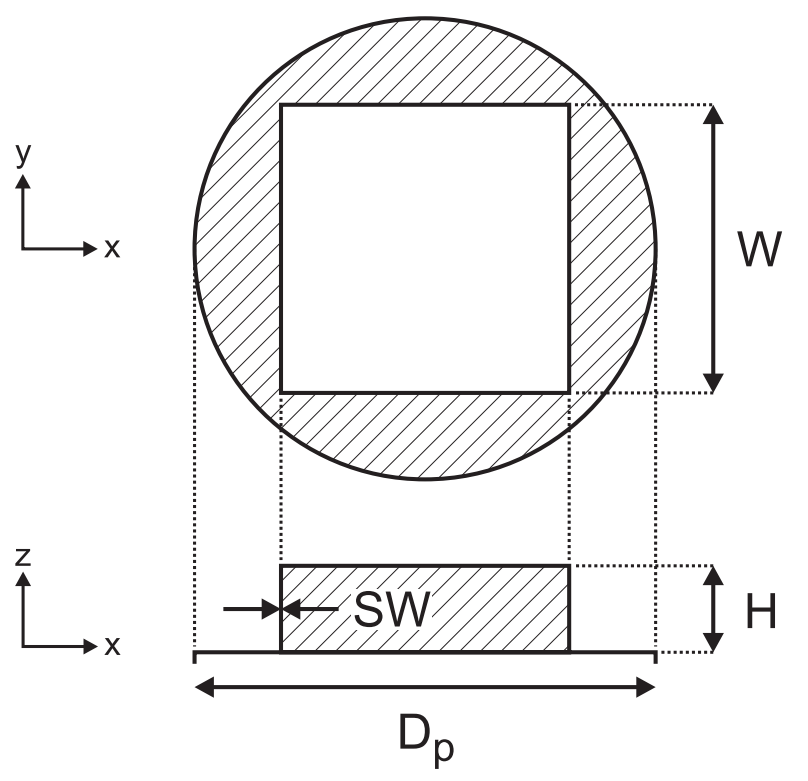

Figure 3.2: Schematic drawing of the convection cell (not to scale). $\mathrm{SW}=$ thickness of the sidewall, $\mathrm{W}=$ width of the cell, $\mathrm{H}=$ height of the cell, $D_{\mathrm{p}}=$ diameter of the bottom of the cell.

convection cell. A detailed description of the $\mathrm{SF}_{6}$ and $\mathrm{He}$ filling procedure is given in Section 3.4. The needle valve NV3 is connected to a vacuum pump (Labor-VakuumSystem LVS 610, ILMVAC GmbH) that is used to evacuate the pressure vessel down to $p \approx 1$ mbar before it is filled with gas. Two ultra precision pressure transducers PG1 and PG2 are used to monitor the system pressure inside the convection apparatus. The pressure gauge PG1 (model Z, Sensotec Inc.) is connected to a 41/2 digit signal conditioner (Sensotec Model GM, Sensotec Inc.) and the pressure gauge PG2 (model STJE, Sensotec Inc.) is read out by a digital multimeter (Keithley 2001 DMM, Keithley Instruments Inc.). A linear regulated high performance power supply (model A10TN110, Acopian Technical Company) is used as a voltage supply for PG2. The read out of the thermistors and the voltages is managed with a ten channel scanner card (model 2001-SCAN scanner card, Keithley Instruments Inc.) together with a Keithley 2001 DMM. A measurement program written in C++ was used for the data acquisition. The temperature regulation for the internal water bath and the 
bottom plate is realized with a software based proportional-integral-derivative (PID) algorithm. More details about the convection apparatus can be found in [18, 56].

\subsection{Optical setup}

Fig. 3.3 illustrates the optical setup which is used to image a horizontal plane in the bulk of the convection cell. A high power light emitting diode $(P=3 \mathrm{~W}, U=12 \mathrm{~V}$, luminous color $6500 \mathrm{~K}$, Conrad Electronic SE) is used as a light source to illuminate the convection cell. The solid red line marks the incoming light beam that passes through a 50/50 beam splitter and through the transparent top plate. The polished

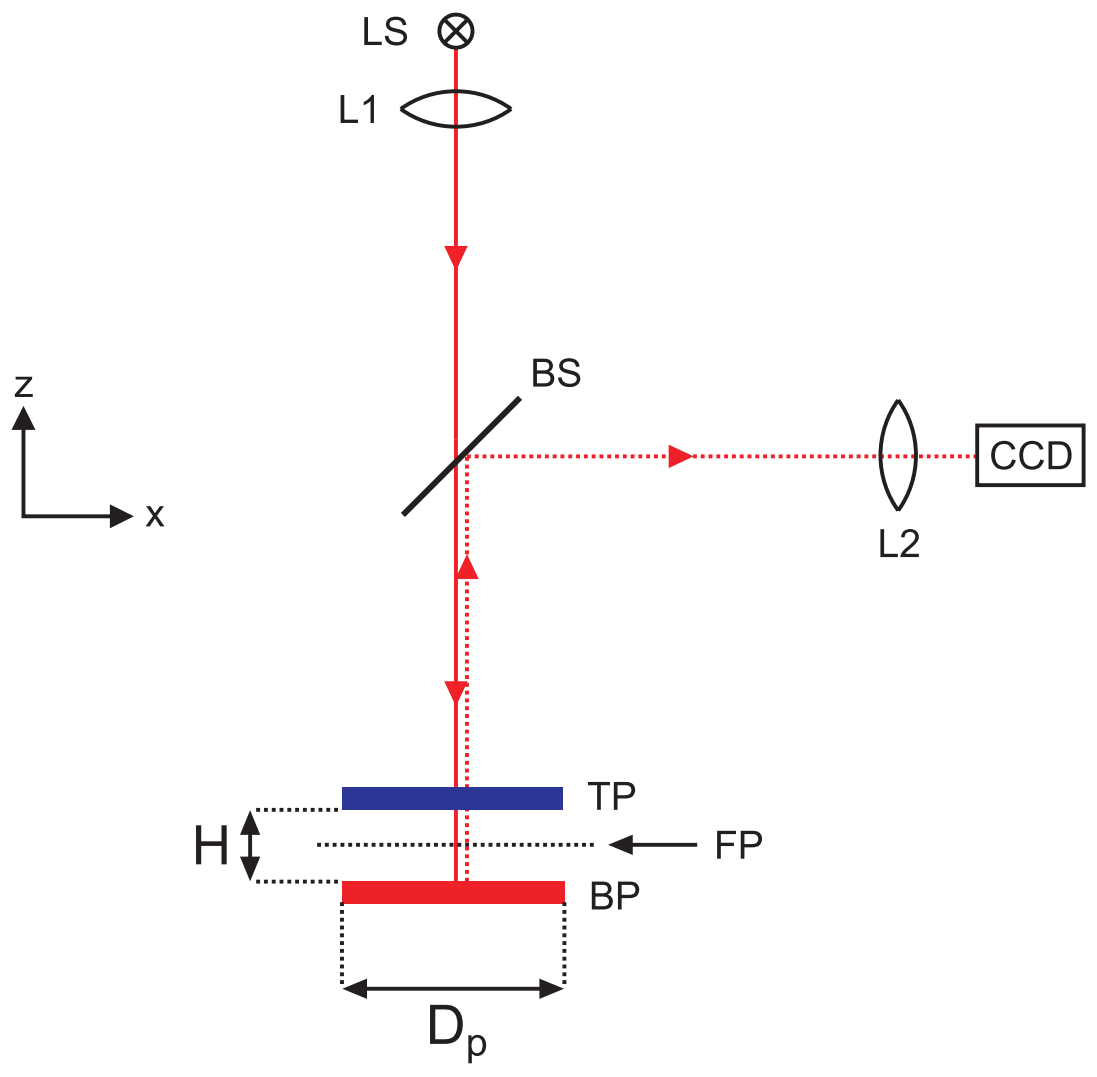

Figure 3.3: Schematic drawing of the optical setup (not to scale). $\mathrm{BP}=$ bottom plate, $\mathrm{BS}=$ beam splitter, $\mathrm{CCD}=$ digital camera, $\mathrm{FP}=$ focal plane, $\mathrm{H}=$ height of the cell, $\mathrm{L} 1$ = lens, $\mathrm{L} 2=$ lens, $\mathrm{LS}=$ light source, $\mathrm{TP}=$ top plate, $\mathrm{D}_{\mathrm{p}}=$ diameter of the bottom and the top plate. 
bottom plate reflects the light back in the direction of the beam splitter as marked by the dashed red line. The reflected light is captured by a macro objective (Tokina AT-X M100 AF Pro D, Kenko Tokina Co. Ltd.) with a focal length of $100 \mathrm{~mm}$ and a maximum aperture of $f / 2.8$. The macro objective is used to image the convection cell and the dashed black line between the bottom and the top plate marks the focal plane of the objective. A monochrome digital CCD camera (QICAM Fast 1394 10bit, QImaging company) records images with a frame rate of $f r=5 \mathrm{fps}$ at full resolution (1360 pixel $\times 1036$ pixel). The camera objective is aligned so that an area of approximately $27 \mathrm{~mm} \times 20 \mathrm{~mm}$ of the central region of the convection cell is mapped onto the CCD.

\subsection{Calibration procedure}

\subsubsection{Temperature calibration}

The thermistors TH1 and TH2 are used to measure the temperature of the internal water bath and the bottom plate respectively. In order to calibrate the thermistors, TH1 is first referenced to a calibrated PT-103 thermistor (Lake Shore Cryotronics Inc.). For this both thermistors are plunged into an aluminum cylinder that serves as a heat mass and a low pass filter for small temperature fluctuations. The water bath of a refrigerated circulator is used to adjust different temperatures to the aluminum cylinder. During the calibration procedure the resistance values of both thermistors are logged. In Fig. 3.4 the resistance of $\mathrm{TH} 1$ is plotted versus the temperature of the calibrated PT-103 thermistor. The open red circles mark the resistance values of TH1 that correspond to the different temperatures that are adjusted with the refrigerated circulator. The solid green line in Fig. 3.4 represents the fit of $R(T)$ with an 


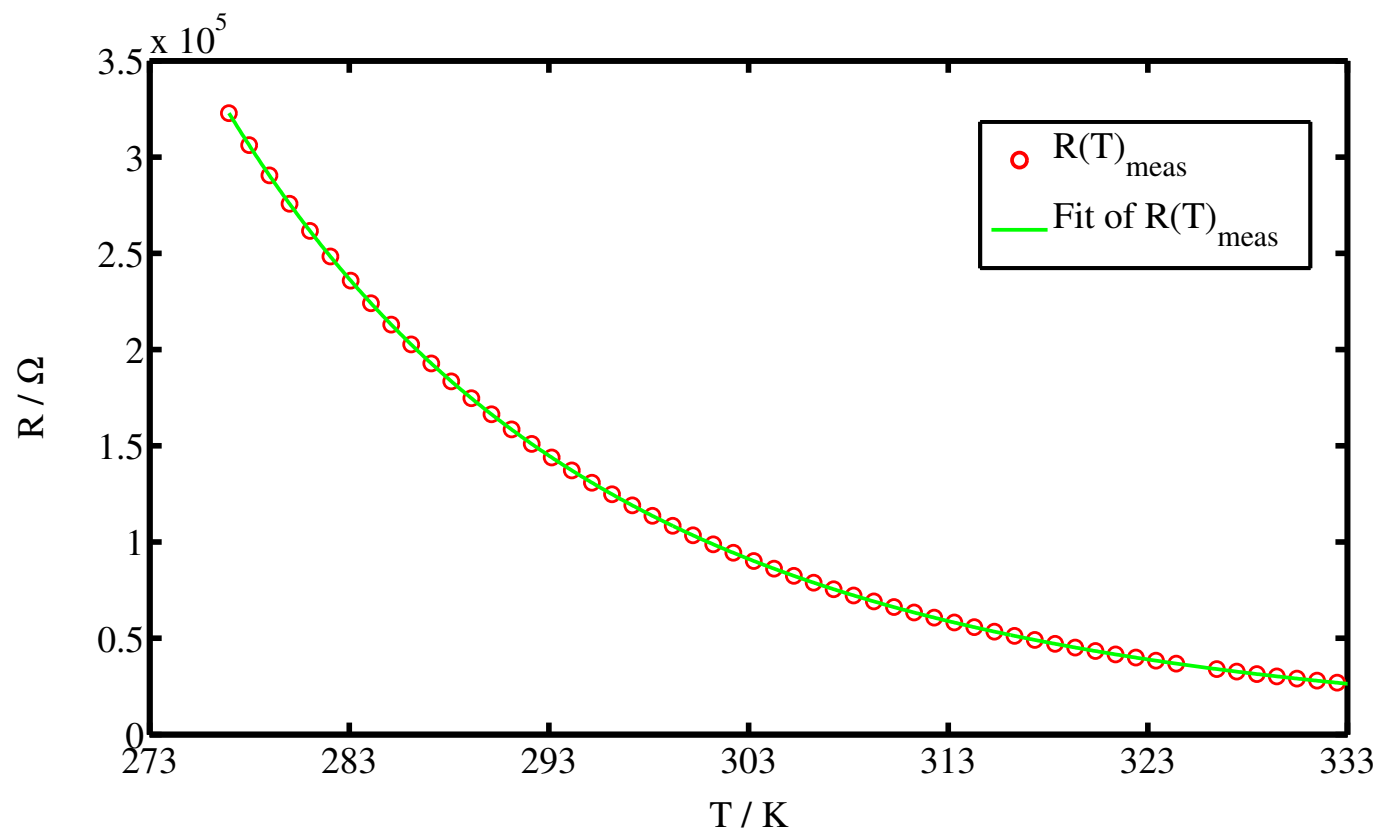

Figure 3.4: Calibration curve $R(T)$ for the thermistor TH1.

\begin{tabular}{lcc}
\hline Parameter & \multicolumn{2}{l}{ Value } \\
\hline$\alpha_{0}$ & $90608.1(7)$ & $\Omega$ \\
$\alpha_{1}$ & $-13.6089(2)$ & \\
$\alpha_{2}$ & $-0.00575(2)$ & $\mathrm{K}^{-1}$ \\
$\alpha_{3}$ & $2.04(5)$ & $\times 10^{-5} \mathrm{~K}^{-2}$ \\
$\alpha_{4}$ & $-1.8(3)$ & $\times 10^{-7} \mathrm{~K}^{-3}$ \\
\hline
\end{tabular}

Table 3.1: Parameters for the fit of $R(T)$ in Fig. 3.4

exponential polynomial of fourth order

$$
R(T)=\alpha_{0} \exp \left[\frac{1}{T}\left(\alpha_{1} \hat{T}+\alpha_{2} \hat{T}^{2}+\alpha_{3} \hat{T}^{3}+\alpha_{4} \hat{T}^{4}\right)\right]
$$

where $\hat{T}=T-T_{0}$, by applying the method of non-linear least squares. The parameters of the fit are listed in Table 3.1. The temperature $T(R)$ for TH1 is determined numerically from Fig. 3.4 by applying the Newton method. 
After the calibration of $\mathrm{TH} 1$, the thermistor $\mathrm{TH} 2$ is referenced to the calibrated thermistor TH1 by performing a calibration in the internal water bath. Before the internal temperature calibration is conducted, the main pressure vessel is thermally insulated by wrapping thick layers of foam around the vessel in order to avoid heat losses to the surroundings. The heating wire is used to regulate the temperature of the internal water bath to the setpoint temperature. The bottom plate adapts to the temperature of the internal water bath. The temperature scenario for the internal temperature calibration is $T_{\mathrm{HE}}<T_{\mathrm{WB}}$, where $T_{\mathrm{HE}}$ and $T_{\mathrm{WB}}$ denote the temperature of the heat exchanger and the internal water bath respectively. The procedure for the calibration of TH2 is the same as for TH1. The calibration curve $R(T)$ is logged by adjusting different temperatures to the internal water bath and by logging the resistance values of TH1 and TH2. The temperature $T(R)$ for TH2 is determined numerically. Both thermistors TH1 and TH2 are measured by using two-terminal sensing.

After the calibration of the thermistors $\mathrm{TH} 1$ and $\mathrm{TH} 2$ is completed, a temperature difference between the bottom and the top plate is adjusted by regulating the temperature of the internal water bath and the bottom plate to a certain setpoint temperature. Two software based PID algorithms are employed in order to control the temperature of the internal water bath and the bottom plate by regulating the voltage supply. A temperature stability of $\pm 1 \mathrm{mK}$ and $\pm 2 \mathrm{mK}$ is achieved for the internal water bath and the bottom plate respectively.

\subsubsection{Pressure calibration}

The pressure transducers PG1 and PG2 are used to determine the system pressure $p$ inside the main pressure vessel. The pressure transducer PG2 is calibrated with an analog pressure gauge (HEISE CM-121691, Ashcroft Inc.). By adjusting different 


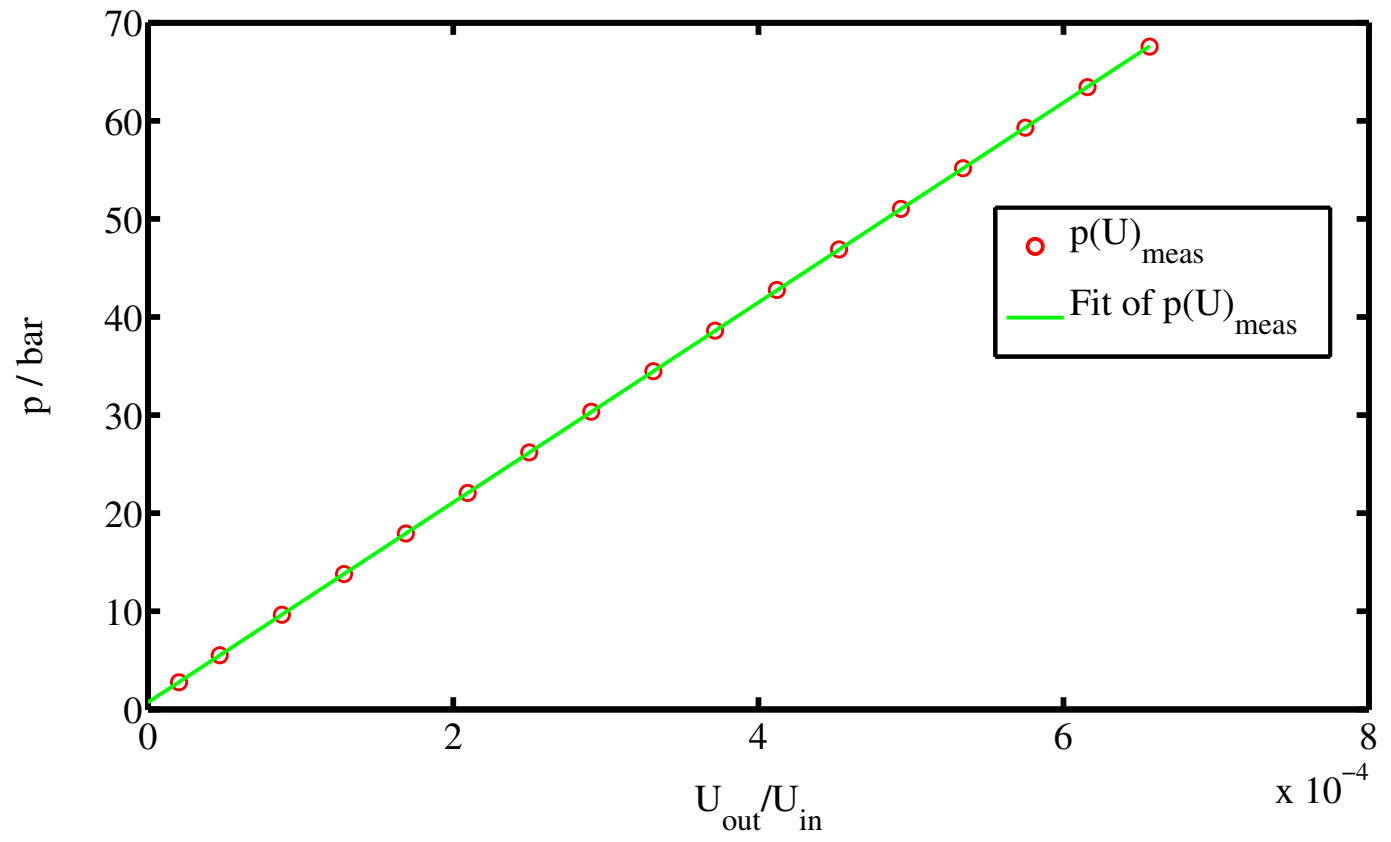

Figure 3.5: Calibration curve $p(U)$ for the pressure transducer PG2.

\begin{tabular}{lll}
\hline Parameter & Value & \\
\hline$\beta_{0}$ & $0.71(1)$ & bar \\
$\beta_{1}$ & $1.01913(3) \times 10^{5}$ & bar \\
\hline
\end{tabular}

Table 3.2: Parameters for the fit of $p(U)$ in Fig. 3.5 .

pressures, the input voltage $U_{\text {in }}$ of the Acopian power supply and the output voltage $U_{\text {out }}$ of the pressure transducer PG2 are logged. In Fig. 3.5 the pressure of the analog gauge is plotted versus the voltage ratio $U_{\text {out }} / U_{\text {in }}$. The open red circles mark the values of the voltage ratio that correspond to the different pressure values. The solid green line in Fig. 3.5 represents the fit of $p(U)$ with a polynomial of first order

$$
p(U)=\beta_{0}+\beta_{1}\left(\frac{U_{\text {out }}}{U_{\text {in }}}\right),
$$


by applying the method of non-linear least squares. The parameters of the fit are listed in Table 3.2 .

\subsubsection{Plate alignment}

The top plate can be aligned with respect to the bottom plate by employing a system of three screw drivers which can be operated from outside the main pressure vessel. This allows for the alignment of the top plate even if the convection apparatus is under pressure. Both plates are aligned as parallel as possible so that the cell height is uniform throughout the convection cell. As shown in Fig. 3.6, both plates are aligned if only higher order deformations remain and no stripe pattern with a preferential orientation is visible. The interference patterns in Fig. 3.6 result from the illumination with a He-Ne laser.

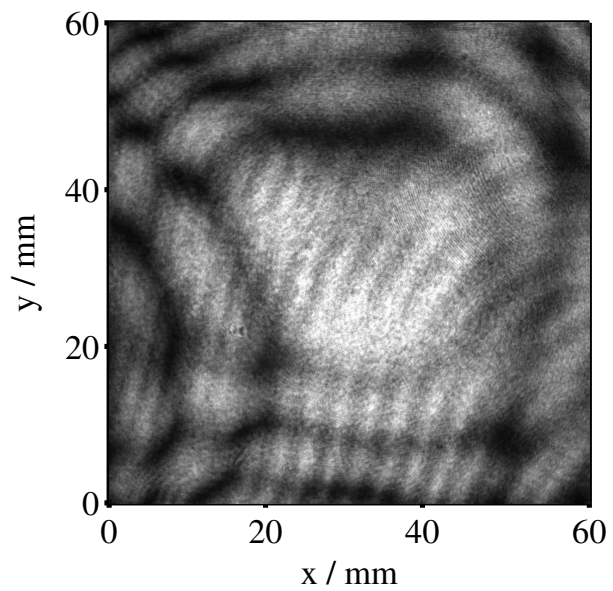

(a)

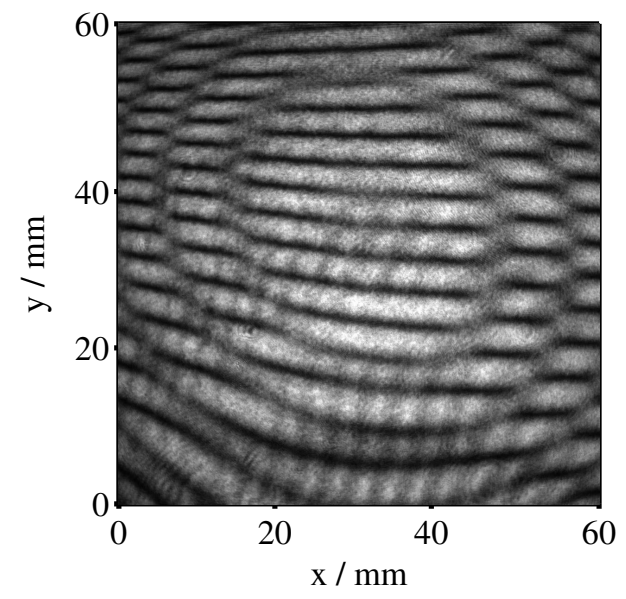

(b)

Figure 3.6: Alignment of the bottom and the top plate. In (a) both plates are aligned parallel to each other so that the interference pattern shows no preferred direction. In (b) the top plate is slightly tilted in the $y$-direction and a stripe pattern appears that is oriented in the direction in which the top plate is tilted. 


\subsubsection{Temperature correction}

Since the bottom and the top plate have a finite thermal conductivity and a thickness of nearly $1 \mathrm{~cm}$, it is conjectured that the temperature at the underside and the topside of the two plates diverges. An estimate for the temperature drop across the bottom and the top plate is made by employing Fourier's law of heat conduction

$$
\mathbf{q}=\lambda \nabla T
$$

Here $\mathbf{q}$ denotes the heat flux and $\lambda$ the thermal conductivity of the plate material [39, 42]. Since the heat input into the top plate is not known, only the temperature drop across the bottom plate is estimated. In order to calculate the temperature difference $\Delta T_{\mathrm{Si}}$ between the underside and the topside of the bottom plate, Eq. (3.4) is written in the form

$$
\begin{aligned}
P & =\lambda_{\mathrm{Si}} A_{\mathrm{p}} \frac{\Delta T_{\mathrm{Si}}}{H_{\mathrm{p}}} \\
\Rightarrow \Delta T_{\mathrm{Si}} & =\frac{P H_{\mathrm{p}}}{\lambda_{\mathrm{Si}} A_{\mathrm{p}}} .
\end{aligned}
$$

Here $P$ is the heating power of the bottom plate heater, $A_{\mathrm{p}}$ the area of the bottom plate, $H_{\mathrm{p}}$ the height of the bottom plate and $\lambda_{\mathrm{Si}}$ the thermal conductivity of silicon. By inserting the values in Table 3.3 into Eq. (3.5), the temperature drop across the bottom plate is determined to

$$
\Delta T_{\mathrm{Si}}=0.002 \mathrm{~K}=2 \mathrm{mK} .
$$

In Section 3.3 .1 it is shown, that the stability of the bottom plate temperature is $\pm 2 \mathrm{mK}$. Since the temperature drop $\Delta T_{\mathrm{Si}}$ across the bottom plate is smaller than the 


\begin{tabular}{ll}
\hline $\begin{array}{l}\text { Parameters } \\
(T=318.1 \mathrm{~K})\end{array}$ & Value \\
\hline Height of the bottom plate $H_{\mathrm{p}}$ & $9.5 \times 10^{-3} \mathrm{~m}$ \\
Area of the bottom plate $A_{\mathrm{p}}$ & $8.1 \times 10^{-3} \mathrm{~m}^{2}$ \\
${ }^{\text {Ta }}$ Thermal conductivity $\lambda_{\mathrm{Si}}(T)$ of silicon & $137 \quad \mathrm{~W} \mathrm{~m}^{-1} \mathrm{~K}^{-1}$ \\
Heating power $P$ & $268 \times 10^{-3} \mathrm{~W}$ \\
\hline
\end{tabular}

${ }^{a}$ Data taken from [28].

Table 3.3: Parameters for the temperature correction of the bottom plate.

fluctuations of the bottom plate temperature, the temperature drop is neglected and it is assumed that the temperature at the underside of the bottom plate equals the temperature at the topside. If the same heat input $P$ is assumed for the top plate and instead the thermal conductivity of sapphire is inserted in Eq. (3.5), then the temperature drop across the sapphire plate $\Delta T_{\mathrm{Sa}}$ is found to be $8 \mathrm{mK}$. However, due to heat losses it can be assumed that the real heat input into the top plate is lower than $P$ what results in a smaller value of $\Delta T_{\mathrm{Sa}}$. Therefore the temperature drop across the top plate is neglected as well and it is assumed that the temperature at the topside equals the temperature at the underside of the top plate.

\subsubsection{Scale calibration}

A periodic stripe pattern is used as a reference scale in order to determine a length scale for the images that were recorded with the digital camera in Fig. 3.3. Black stripes that are equally spaced by $1 \mathrm{~mm}$ were printed on a transparent foil. A $40 \mathrm{~mm} \times 40 \mathrm{~mm}$ piece of the foil is cut out and placed on top of the big sapphire pressure window in Fig. 3.1. The objective of the digital camera is focussed into the convection cell as described in Section 3.2. Therefore the stripe pattern is not in 


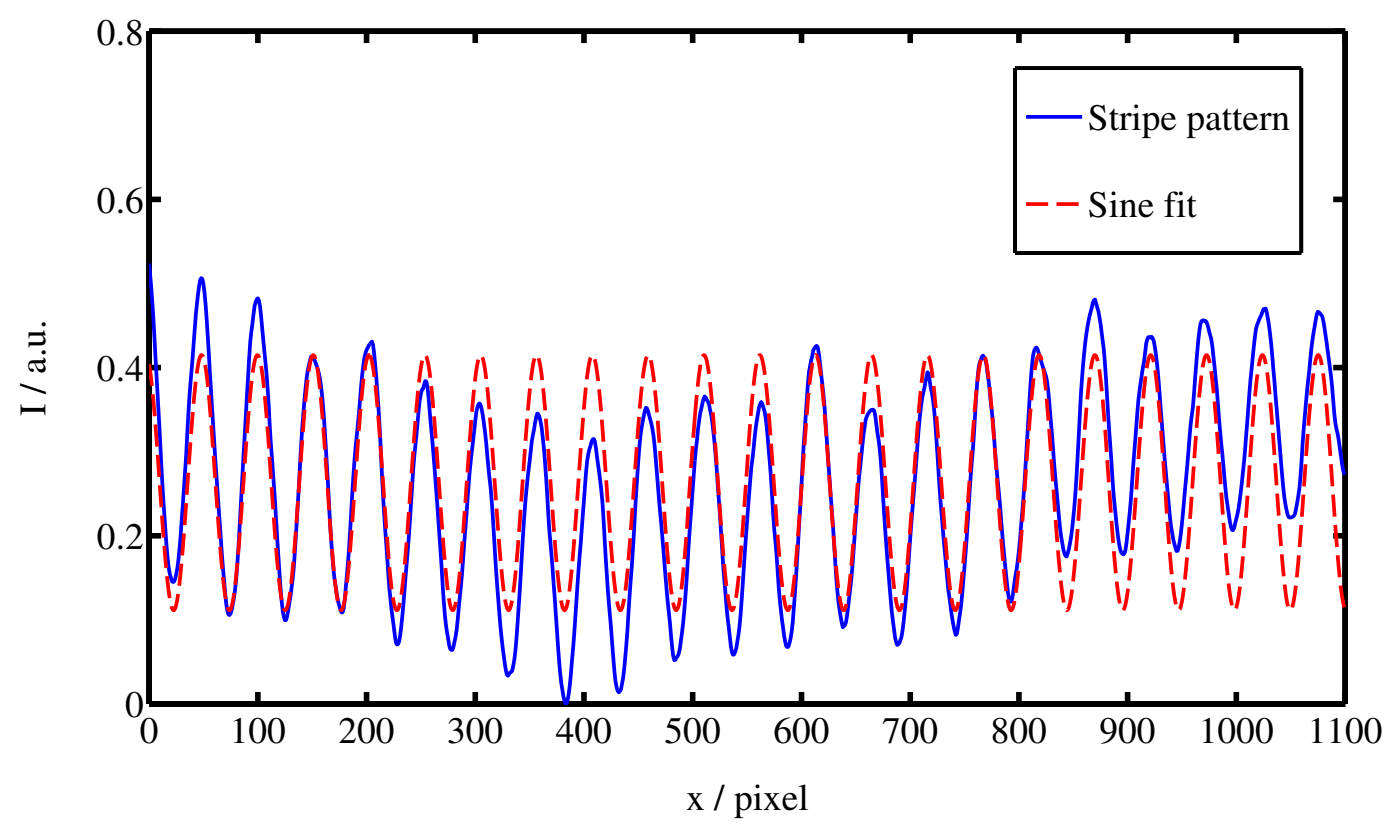

Figure 3.7: Normalized intensity profile $I_{\mathrm{n}}(x)$ for the calibration of the length scale.

\begin{tabular}{ll}
\hline Parameter & Value \\
\hline$\gamma_{0}$ & $0.263(4)$ \\
$\gamma_{1}$ & $0.152(5)$ \\
$\gamma_{2}$ & $51.35(4) \mathrm{pixel}^{-1}$ \\
$\gamma_{3}$ & $-0.88(8)$ \\
\hline
\end{tabular}

Table 3.4: Parameters for the fit of $I_{n}(x)$ in Fig. 3.7.

focus and the peaks of the single stripes are widened and not sharp. Images were taken with and without the strip pattern on top of the sapphire pressure window. By integrating a certain region of the image, the normalized intensity profile $I_{n}(x)$ is determined as follows

$$
I_{\mathrm{n}}(x)=\frac{\sum_{y_{1}}^{y_{2}} I(x, y)}{\sum_{y_{1}}^{y_{2}} I_{0}(x, y)} .
$$

Here $I(x, y)$ and $I_{0}(x, y)$ are the integrated intensity profiles in $x$-direction of an image with and without the reference scale respectively. $y_{1}$ and $y_{2}$ label the number 
of integrated lines in $y$-direction. The solid blue line in Fig. 3.7 shows $I_{\mathrm{n}}(x)$ in $x$ direction for the periodic stripe pattern. Each minimum in Fig. 3.7 marks a black stripe. The dashed red line represents the fit of $I_{\mathrm{n}}(x)$ with a sine function

$$
f(x)=\gamma_{0}+\gamma_{1} \sin \left(\gamma_{2} x-\gamma_{3}\right),
$$

by applying the method of non-linear least squares. The parameters of the fit are listed in Table 3.4. The wavelength $\lambda=2 \pi / \gamma_{2}$ specifies the distance between two consecutive peaks which corresponds to the spacing between two stripes. If the wavelength $\lambda$ is known, a conversion factor $s=\mathrm{mm} /$ pixel can be determined in order to convert the pixel-scale into a mm-scale.

\subsection{Filling procedure for $\mathrm{SF}_{6}$ and $\mathrm{He}$}

\subsubsection{Filling procedure for $\mathrm{SF}_{6}$}

Fig. 3.8 illustrates the filling procedure for $\mathrm{SF}_{6}$ into the main pressure vessel. The mass fraction of $\mathrm{SF}_{6}$ must be adjusted in such a way that a liquid and a vapor phase of $\mathrm{SF}_{6}$ coexist in the convection cell. Since the experiments are conducted at temperatures close to the critical temperature $T_{\mathrm{c}}$ of $\mathrm{SF}_{6}$, the pressure vessel must contain a proper amount of liquid $\mathrm{SF}_{6}$ so that the liquid phase of $\mathrm{SF}_{6}$ can be heated up to a temperature close to $T_{\mathrm{c}}$. The vapor pressure of $\mathrm{SF}_{6}$ is determined by the LVCL in Fig. 2.2. If only a minor amount of liquid $\mathrm{SF}_{6}$ is contained in the pressure vessel and the temperature is increased, then the liquid phase of $\mathrm{SF}_{6}$ starts to evaporate and the vapor pressure increases. If the entire liquid phase of $\mathrm{SF}_{6}$ is converted into a gas, then a pure gaseous phase of $\mathrm{SF}_{6}$ exists in the pressure vessel. If the temperature is further increased, then the gaseous phase of $\mathrm{SF}_{6}$ behaves like an ideal gas. In this case more 


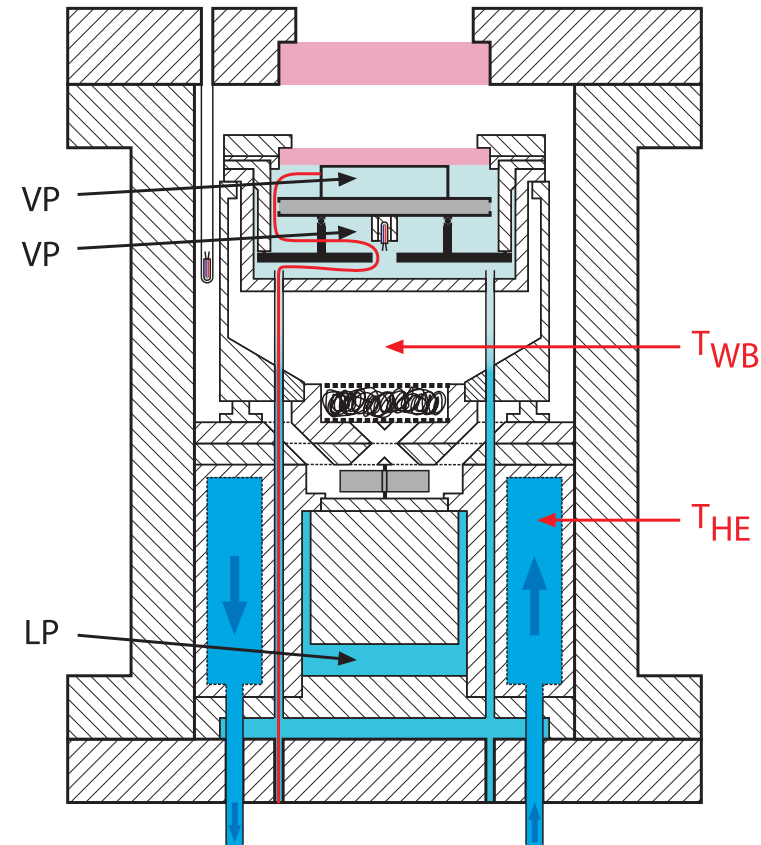

(a)

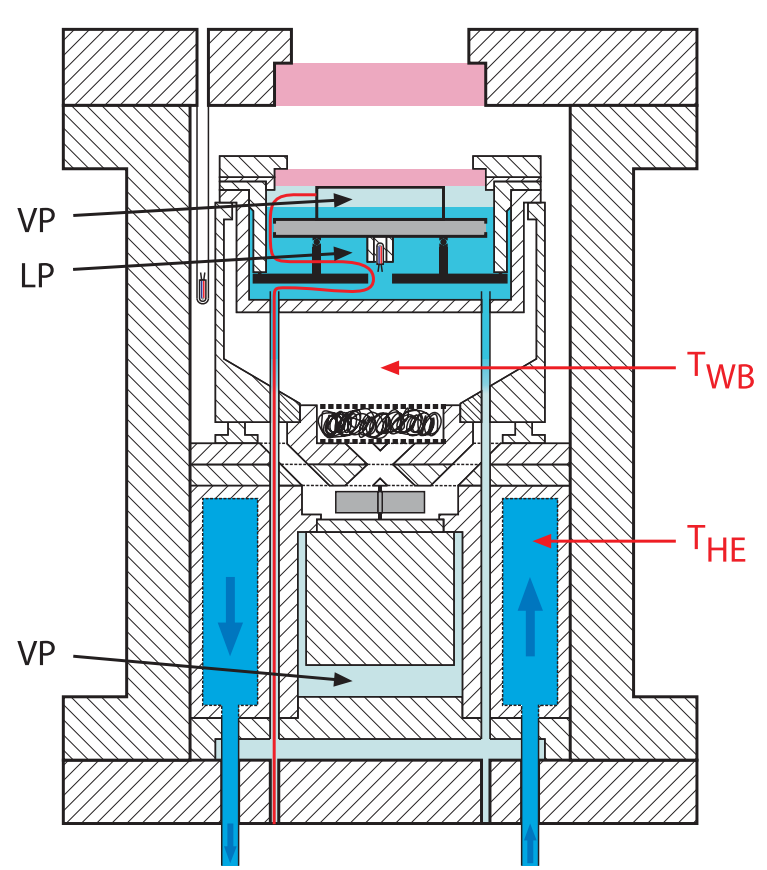

(b)

Figure 3.8: Schematic drawing of the $\mathrm{SF}_{6}$ filling procedure. $\mathrm{LP}=$ liquid phase, $\mathrm{VP}=$ vapor phase, $T_{\mathrm{HE}}=$ temperature of the heat exchanger, $T_{\mathrm{RT}}=$ room temperature, $T_{\mathrm{WB}}$ = temperature of the internal water bath. (a) shows the scenario when $T_{\mathrm{HE}}<T_{\mathrm{WB}}<$ $T_{\mathrm{RT}}$. (b) shows the scenario when $T_{\mathrm{HE}}>T_{\mathrm{WB}}>T_{\mathrm{RT}}$.

liquid $\mathrm{SF}_{6}$ must be filled into the pressure vessel. On the other hand if too much liquid $\mathrm{SF}_{6}$ is contained in the pressure vessel and the temperature is increased, then the liquid phase of $\mathrm{SF}_{6}$ starts to evaporate and the vapor pressure of $\mathrm{SF}_{6}$ increases. The liquid phase of $\mathrm{SF}_{6}$ also expands since the density of liquid $\mathrm{SF}_{6}$ decreases with increasing temperature and it can happen that the liquid phase of $\mathrm{SF}_{6}$ fills the entire volume of the pressure vessel before the desired working temperature is reached. In this case $\mathrm{SF}_{6}$ must be released from the pressure vessel or the filling procedure must be repeated from the beginning. The filling procedure for $\mathrm{SF}_{6}$ can be divided up into two steps: 
1. Cooling procedure:

(a) First the pressure vessel must be cooled below the room temperature $T_{\mathrm{RT}}$ so that the gaseous $\mathrm{SF}_{6}$ from the gas bottle starts to liquefy inside the pressure vessel. Therefore the temperature of the refrigerated circulator is adjusted to $T_{\mathrm{HE}}=279.15 \mathrm{~K}$. The refrigerated circulator cools the heat exchanger which in turn cools the pressure vessel. The pressure vessel is cooled until the temperature of the internal water bath $T_{\mathrm{WB}}$ is below the room temperature.

(b) If $T_{\mathrm{HE}}<T_{\mathrm{WB}}<T_{\mathrm{RT}}$, then the needle valve NV2 in Fig. 3.1 is opened to fill the pressure vessel with gaseous $\mathrm{SF}_{6}$ from the gas bottle. The temperature of the internal water bath is larger than the temperature of the heat exchanger. That is why the liquid phase of $\mathrm{SF}_{6}$ starts to condense in the lower volume of the pressure vessel. The scenario of the cooling procedure is illustrated in Fig. 3.8a.

(c) If the lower volume is filled with liquid $\mathrm{SF}_{6}$, then the condensation also sets in at the top plate of the convection cell. At this point enough liquid $\mathrm{SF}_{6}$ is contained in the pressure vessel and the needle valve NV2 is closed.

2. Heating procedure:

(a) If enough liquid $\mathrm{SF}_{6}$ is contained in the pressure vessel, then the temperature of the heat exchanger is increased to a value below the desired working temperature. The liquid phase of $\mathrm{SF}_{6}$ starts to evaporate in the lower volume of the pressure vessel and the vapor pressure of $\mathrm{SF}_{6}$ increases. The liquid phase of $\mathrm{SF}_{6}$ expands since the density of liquid $\mathrm{SF}_{6}$ decreases with increasing temperature. The scenario of the heating procedure is illustrated in Fig. $3.8 \mathrm{~b}$. The liquid phase of $\mathrm{SF}_{6}$ begins to condense in the upper volume of the pressure vessel since the temperature of the internal water bath is lower than the temperature of the heat exchanger that means $T_{\mathrm{HE}}>T_{\mathrm{WB}}>T_{\mathrm{RT}}$. This 
temperature scenario is only possible because of heat losses due to thermal radiation. As already mentioned, care must be taken when filling liquid $\mathrm{SF}_{6}$ into the pressure vessel. If too much liquid $\mathrm{SF}_{6}$ is contained in the pressure vessel, then the liquid phase of $\mathrm{SF}_{6}$ fills the entire volume of the pressure vessel before the desired working temperature is reached. In this case the needle valve NV3 must be opened in order to release $\mathrm{SF}_{6}$ from the pressure vessel.

(b) If a liquid and a vapor phase of $\mathrm{SF}_{6}$ coexist in the convection cell, then the water bath heater is switched on and the internal water bath is heated to the desired setpoint temperature. During the experiments $T_{\mathrm{HE}}$ is kept constant and only $T_{\mathrm{WB}}$ is varied. By switching the bottom plate heater on and off, the evaporation and the condensation of $\mathrm{SF}_{6}$ can be controlled.

The heat exchanger is used to regulate the level of liquid $\mathrm{SF}_{6}$ in the convection cell. The liquid phase of $\mathrm{SF}_{6}$ fills about half the height of the convection cell. If the level of liquid $\mathrm{SF}_{6}$ in the convection cell drops, then $T_{\mathrm{HE}}$ must be increased in order to push more $\mathrm{SF}_{6}$ into the upper volume of the pressure vessel as shown in Fig. 3.8b. On the other hand if the level of liquid $\mathrm{SF}_{6}$ in the convection cell is too large, then $T_{\mathrm{HE}}$ must be decreased in order to reduce the amount of liquid $\mathrm{SF}_{6}$ in the upper volume.

Fig. 3.9 illustrates the heat flow in the main pressure vessel. $Q_{R C}$ denotes the input and output of heat by the refrigerated circulator, $Q_{\mathrm{WBH}}$ the heat input by the water bath heater and $Q_{\mathrm{BPH}}$ the heat input by the bottom plate heater. The heat losses are labeled by $Q_{\mathrm{L}}$. The main input and output of heat $Q_{\mathrm{RC}}$ is provided by the refrigerated circulator that is connected to the heat exchanger. The refrigerated circulator has a maximum heating power of $2000 \mathrm{~W}$. The heat exchanger heats and cools the water bath. Since the pressure vessel is not thermally insulated, for the scenario $T_{\mathrm{HE}}>T_{\mathrm{WB}}>T_{\mathrm{RT}}$ heat losses $Q_{\mathrm{L}}$ occur from the heat exchanger and the water bath 


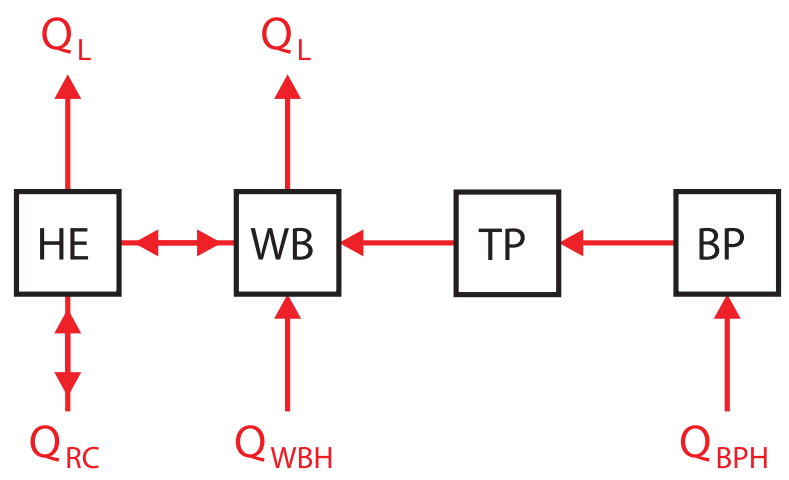

Figure 3.9: Schematic diagram of the heat flow in the main pressure vessel. $\mathrm{BP}=$ bottom plate, $\mathrm{HE}=$ heat exchanger, $\mathrm{TP}=$ top plate, $\mathrm{WB}=$ internal water bath.

to the surroundings. Another source of heat input is the water bath heater and the bottom plate heater which supply a heating power of $120 \mathrm{~W}$ and $360 \mathrm{~W}$ respectively. Since the bottom plate is thermally insulated from the can, the heat is transported via conduction and convection from the bottom to the top plate and is then transferred to the water bath. The heat input of the water bath heater and the bottom plate heater is then removed by the refrigerated circulator.

\subsubsection{Filling procedure for $\mathrm{He}$}

If a liquid and a vapor phase of $\mathrm{SF}_{6}$ coexist in the convection cell, then the needle valve NV1 in Fig. 3.1 is carefully opened and a small amount of He is filled into the convection cell via the teflon tube. The gaseous phase of He mixes with the existing vapor phase of $\mathrm{SF}_{6}$ and a mixture with a high concentration of $\mathrm{He}$ and a low concentration of $\mathrm{SF}_{6}$ forms. An estimate for the turbulent mixing time is given in Section A.4. The result in Eqs. A.16a and A.16b shows that the mixing process of the two gases takes less than $1 \mathrm{~min}$. Due to the large concentration of He in the gaseous phase, a concentration gradient builds up between the liquid phase of $\mathrm{SF}_{6}$ and the gaseous phase of $\mathrm{SF}_{6}$ and He. The concentration of $\mathrm{He}$ in the gaseous phase of $\mathrm{SF}_{6}$ and $\mathrm{He}$ is larger than in the liquid phase of $\mathrm{SF}_{6}$ and as a consequence He starts to 
diffuse into the liquid phase of $\mathrm{SF}_{6}$. Therefore the concentration of He in the liquid phase of $\mathrm{SF}_{6}$ is increasing and the concentration of He in the gaseous phase of $\mathrm{SF}_{6}$ and He is decreasing over time. On the other hand if $\mathrm{He}$ is filled into the convection cell, the concentration of $\mathrm{SF}_{6}$ in the gaseous phase of $\mathrm{SF}_{6}$ and He is reduced. Therefore the liquid phase of $\mathrm{SF}_{6}$ begins to evaporate and the concentration of $\mathrm{SF}_{6}$ in the gaseous phase of $\mathrm{SF}_{6}$ and He is increasing until the gaseous phase of $\mathrm{SF}_{6}$ and He is again saturated with $\mathrm{SF}_{6}$ vapor. Therefore, whenever He is filled into the convection cell, the liquid phase of $\mathrm{SF}_{6}$ is reduced since more and more liquid $\mathrm{SF}_{6}$ is converted into a vapor. If the concentration of $\mathrm{He}$ is too large, then the entire liquid phase of $\mathrm{SF}_{6}$ is converted into a vapor and only a pure gaseous phase of $\mathrm{SF}_{6}$ and He exists in the pressure vessel. Therefore it is advised to keep the concentration of He as low as possible so that a liquid phase of $\mathrm{SF}_{6}$ always exists above the bottom plate.

\subsubsection{Temperature scenarios for the main pressure vessel}

Table 3.5 summarizes the effects if the temperature of the heat exchanger is changed.

Three different temperature scenarios are distinguished. For the filling procedure of $\mathrm{SF}_{6}$, the temperature scenario is $T_{\mathrm{HE}}<T_{\mathrm{WB}}<T_{\mathrm{RT}}<T_{\mathrm{C}}$. A liquid phase of $\mathrm{SF}_{6}$ forms

\begin{tabular}{ll}
\hline Condition & Effect \\
\hline$T_{\mathrm{HE}}<T_{\mathrm{WB}}<T_{\mathrm{RT}}<T_{\mathrm{C}}$ & $\begin{array}{l}\text { The liquid phase of } \mathrm{SF}_{6} \text { condenses in the lower volume } \\
\text { and a vapor phase of } \mathrm{SF}_{6} \text { remains in the upper volume. }\end{array}$ \\
\hline$T_{\mathrm{C}}>T_{\mathrm{HE}}>T_{\mathrm{WB}}>T_{\mathrm{RT}}$ & $\begin{array}{l}\text { The liquid phase of } \mathrm{SF}_{6} \text { condenses in the lower and the } \\
\text { upper volume. }\end{array}$ \\
\hline$T_{\mathrm{HE}}>T_{\mathrm{C}}>T_{\mathrm{WB}}>T_{\mathrm{RT}}$ & $\begin{array}{l}\text { The liquid phase of } \mathrm{SF}_{6} \text { condenses in the upper volume } \\
\text { and a vapor phase of } \mathrm{SF}_{6} \text { remains in the lower volume. }\end{array}$ \\
\hline
\end{tabular}

Table 3.5: Effects of changing the temperature of the heat exchanger. 
predominantly in the lower volume of the pressure vessel where the temperature is the lowest and a vapor phase of $\mathrm{SF}_{6}$ remains in the upper volume. On the other hand if the temperature scenario is $T_{\mathrm{c}}>T_{\mathrm{HE}}>T_{\mathrm{WB}}>T_{\mathrm{RT}}$, then a liquid phase of $\mathrm{SF}_{6}$ forms in the lower as well as in the upper volume of the pressure vessel. For the experiments that are described in Chapter 4 , the temperature scenario was $T_{\mathrm{HE}}>$ $T_{\mathrm{c}}>T_{\mathrm{WB}}>T_{\mathrm{RT}}$. In this case a liquid phase of $\mathrm{SF}_{6}$ predominantly condenses in the upper volume of the pressure vessel and a vapor phase of $\mathrm{SF}_{6}$ remains in the lower volume. 


\section{Chapter 4}

\section{Results}

\subsection{General remarks and observations}

This section describes general observations that were made when the $\mathrm{SF}_{6}$ and $\mathrm{He}$ mixture was investigated. During the experiments a liquid phase of $\mathrm{SF}_{6}$ and a gaseous phase of $\mathrm{SF}_{6}$ and $\mathrm{He}$ coexisted in the convection cell. The liquid phase of $\mathrm{SF}_{6}$ filled about half the height of the convection cell. Throughout the experiments the temperature of the heat exchanger $T_{\mathrm{HE}}$ was kept constant. The temperature scenario in the pressure vessel was $T_{\mathrm{HE}}>T_{\mathrm{C}}>T_{\mathrm{WB}}>T_{\mathrm{RT}}$ as shown in Table 3.5 .

When the bottom plate heater is switched on, two intriguing observations were made. First a film condensation sets in on the underside of the top plate which results in the formation of a very regular hexagonal droplet pattern. Second cloud-like patterns appear in a thin layer above the liquid-vapor interface and remain stable during the observed time interval. The results of the analysis of the droplet pattern and the cloud-like patterns are presented in Section 4.2 and in Section 4.3 respectively. The droplet pattern and the cloud-like patterns were observed under the experimental conditions that are listed in Table 4.1 


\begin{tabular}{lc}
\hline Experimental parameters & Value \\
\hline Temperature of the top plate $T_{\mathrm{t}}$ & $317.650(1) \mathrm{K}$ \\
Temperature of the bottom plate $T_{\mathrm{b}}$ & $318.101(2) \mathrm{K}$ \\
Temperature difference $\Delta T$ & $0.451(1) \mathrm{K}$ \\
Temperature of the heat exchanger $T_{\mathrm{HE}}$ & $323.15(1) \mathrm{K}$ \\
Pressure $p$ & $53.974(3) \mathrm{bar}$ \\
Molar concentration of $\mathrm{He} y_{2}$ & $14.5 \%$ \\
\hline
\end{tabular}

Table 4.1: Experimental parameters for the observation of the droplet pattern and the cloud-like patterns.

\subsubsection{Thermal diffusion}

Two software based PID algorithms are employed in order to keep the internal water bath and the bottom plate at a constant temperature. A temperature difference is applied between the bottom and the top plate by switching the bottom plate heater on. First the time it takes to heat the bottom plate to a stable temperature is determined. Fig. 4.1 shows the temperature of the bottom plate versus time when the bottom plate heater is switched on. The solid blue and the solid red line in Fig. 4.1 represent the temperature of the top and the bottom plate respectively. The bottom plate heater is switched on at $t_{0}=0$. After an overshooting at the beginning, the temperature of the bottom plate is asymptotically approaching the setpoint value. Despite the overshooting at the beginning, the attempt is made to fit the heating curve in order to estimate the time it takes to heat up the bottom plate. The dashed green line in Fig. 4.1 represents the fit of $T_{\mathrm{b}}(t)$ with an exponential function of the type

$$
T(t)=\delta_{0}+\delta_{1} \exp \left(-\frac{t}{\delta_{2}}\right)
$$




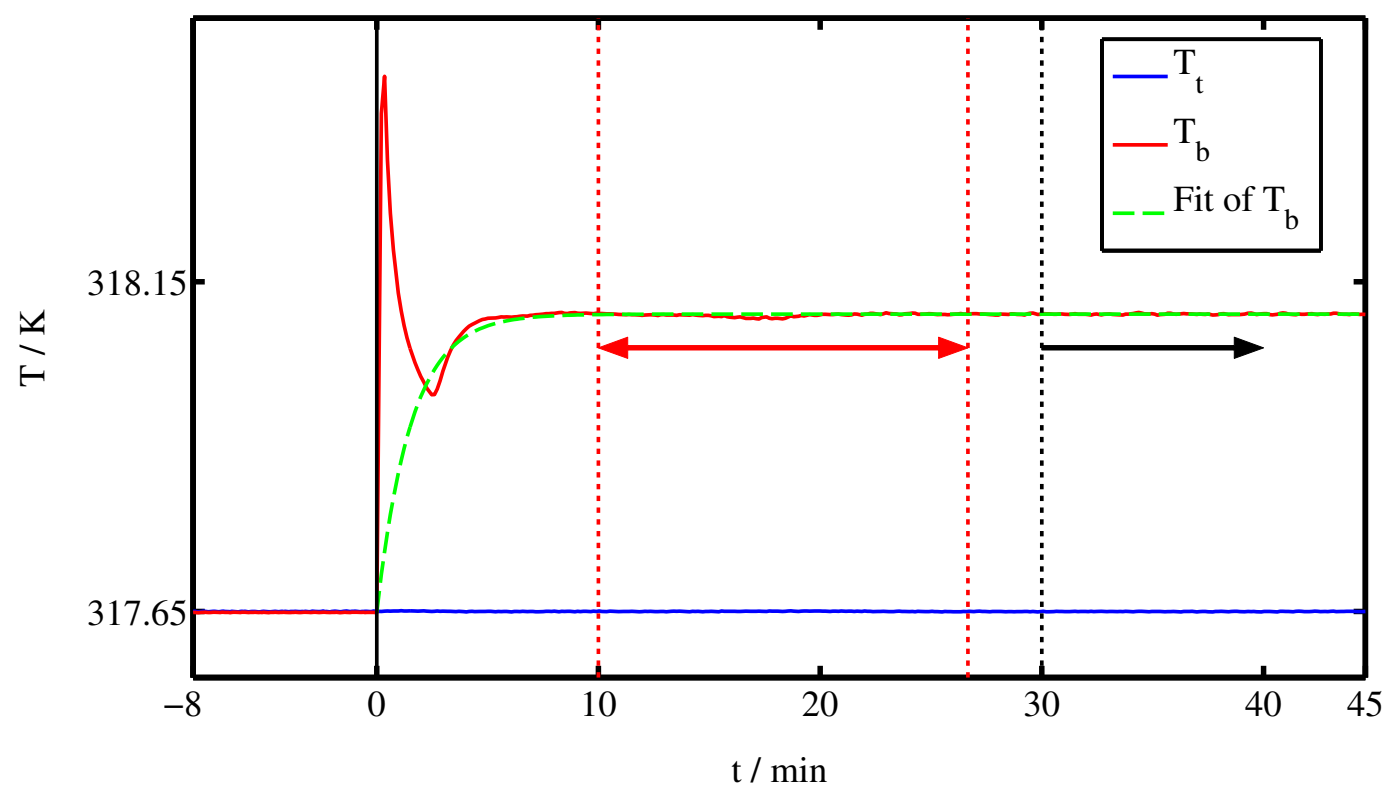

Figure 4.1: Heating curve of the bottom plate. $T_{\mathrm{t}}$ and $T_{\mathrm{b}}$ denote the temperature of the top and the bottom plate respectively. The bottom plate heater is switched on at $t_{0}=0$.

\begin{tabular}{lcc}
\hline Parameter & Value & \\
\hline$\delta_{0}$ & 318.10 & $\mathrm{~K}$ \\
$\delta_{1}$ & -0.45 & $\mathrm{~K}$ \\
$\delta_{2}$ & $1.54(3) \mathrm{min}$ \\
\hline
\end{tabular}

Table 4.2: Parameters for the fit of the heating curve in Fig. 4.1.

for the time interval $3 \mathrm{~min}<t \leq 45 \mathrm{~min}$, by applying the method of non-linear least squares. The time interval $0 \leq t \leq 3 \mathrm{~min}$ where the overshooting occurs is not considered for the fit. Eq. (4.1) is used as a simple model in order to estimate the time it takes to heat the bottom plate to a stable temperature. The parameters of the fit are listed in Table 4.2. The fitted parameters are labeled with an error in parenthesis. The time constant $\tau_{\text {on }}$ for the heating of the bottom plate is found to be $\tau_{\text {on }} \approx 1.5 \mathrm{~min}$. The waiting time $\tau_{\mathrm{on}_{\text {wait }}}$ in order to adjust a stable bottom plate temperature is estimated to $\tau_{\mathrm{on}_{\text {wait }}} \approx 10 \mathrm{~min}$. 


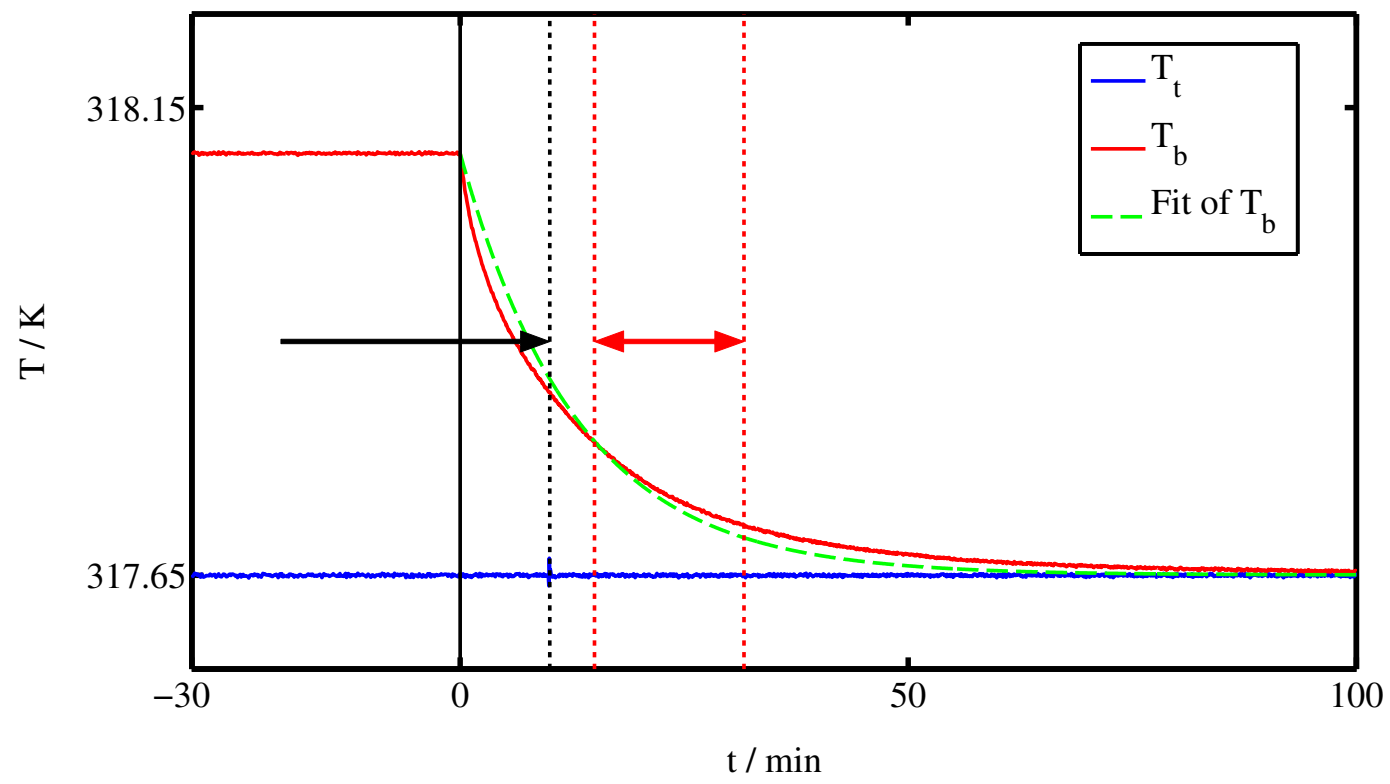

Figure 4.2: Relaxation curve of the bottom plate. $T_{\mathrm{t}}$ and $T_{\mathrm{b}}$ denote the temperature of the top and the bottom plate respectively. The bottom plate heater is switched off at $t_{0}=0$.

\begin{tabular}{lcc}
\hline Parameter & Value & \\
\hline$\delta_{0}$ & 317.65 & $\mathrm{~K}$ \\
$\delta_{1}$ & 0.45 & $\mathrm{~K}$ \\
$\delta_{2}$ & $13.06(7) \mathrm{min}$ \\
\hline
\end{tabular}

Table 4.3: Parameters for the fit of the relaxation curve in Fig. 4.2

The reverse scenario when the bottom plate heater is switched off is shown in Fig. 4.2. The solid blue and the solid red line represent the temperature of the top and the bottom plate respectively. The bottom plate heater is switched off at $t_{0}=0$. The temperature of the bottom plate slowly approaches the temperature of the top plate. The dashed green line in Fig. 4.2 represents the fit of $T_{\mathrm{b}}(t)$ with Eq. (4.1), for the time interval $0 \leq t \leq 280 \mathrm{~min}$, by applying the method of non-linear least squares. The parameters of the fit are listed in Table 4.3 . The time constant $\tau_{\text {off }}$ for the relaxation of the bottom plate temperature is found to be $\tau_{\text {off }} \approx 13 \mathrm{~min}$. The waiting time 
$\tau_{\text {off }_{\text {wait }}}$ until the bottom plate has reached the temperature equilibrium is estimated to $\tau_{\text {off }_{\text {wait }}} \approx 1 \mathrm{~h}$. The relaxation time $\tau_{\text {off }}$ is larger than $\tau_{\text {on }}$ since $\tau_{\text {off }}$ only depends on the thermal properties of the convection cell. The heating time $\tau_{\text {on }}$ not only depends on the thermal properties of the convection cell but also on the heating power $P$ of the bottom plate heater. A larger $P$ shortens the time for the heating of the bottom plate.

\subsubsection{Mass diffusion}

Suppose an one-component system forms a liquid and a vapor phase and is enclosed in a container. If the temperature of the container and of the liquid and the vapor phase are equal, then the system is in a thermal equilibrium. If the vapor phase is in addition in a saturated state and the number of molecules in the vapor phase is equal, then the system is also in a concentration equilibrium. That means the number of molecules that escape from the liquid into the vapor phase equals the number of molecules that change from the vapor into the liquid phase. A system is in a thermodynamic equilibrium if it is in a thermal as well as in a concentration equilibrium at the same time.

Now suppose a two-component system is composed of a component 1 that forms a liquid and a vapor phase and a component 2 that remains in the gaseous phase. The system is again enclosed in a container. If the temperature of the container and of the liquid and the vapor phase are equal, then the system is in a thermal equilibrium. However, it could be even though the number of molecules in the gaseous phase of the two-component system is constant, that the concentration of component 2 in the liquid and the gaseous phase is not in equilibrium. That means the system is not in a concentration equilibrium since more molecules of the component 2 change from the gaseous into the liquid phase than the other way round. This would happen if 


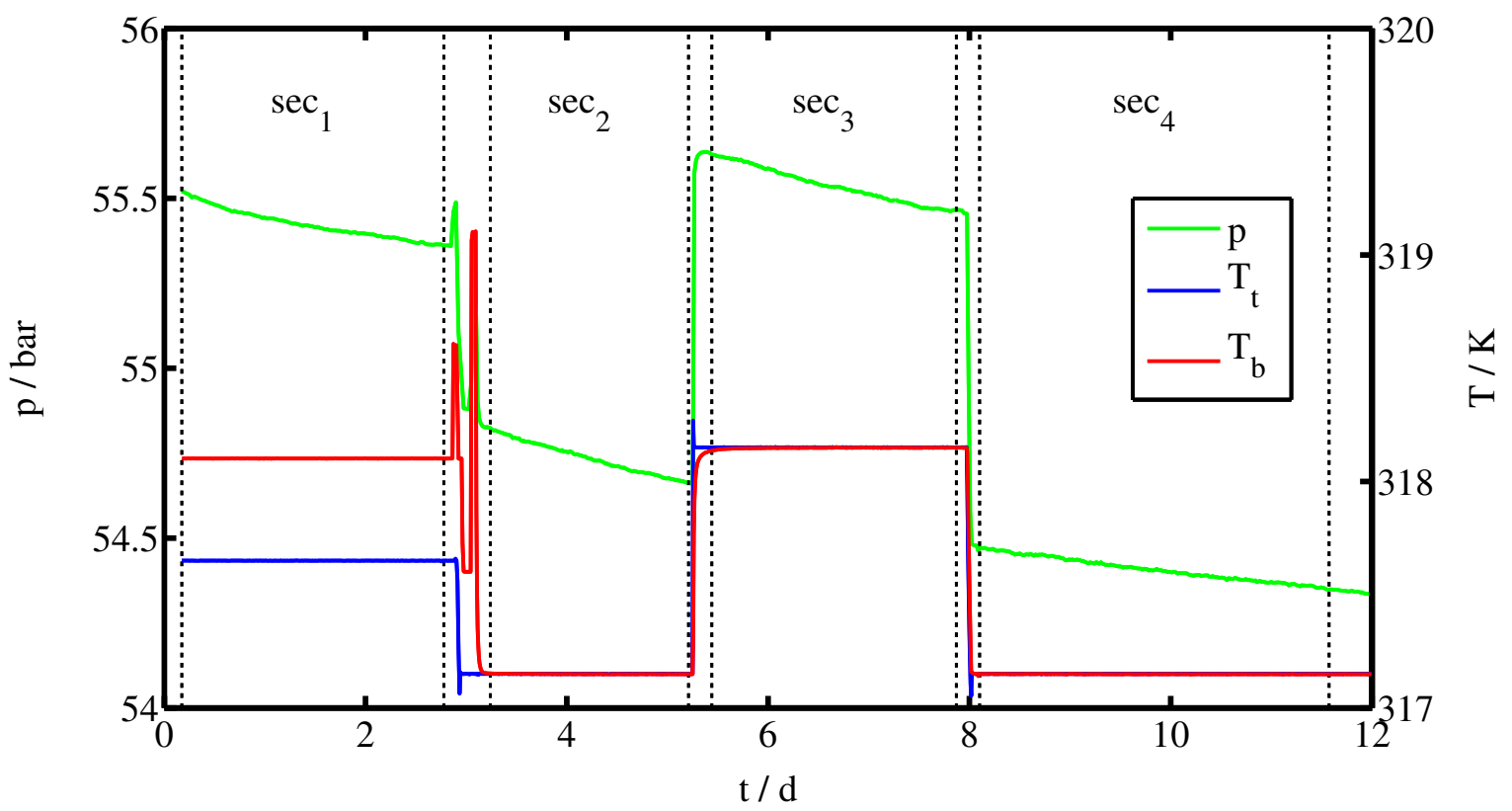

Figure 4.3: Temperature and pressure in the convection apparatus over a time period of approximately $16 \mathrm{~d} . T_{\mathrm{t}}, T_{\mathrm{b}}$ and $p$ denote the temperature of the top plate, the bottom plate and the pressure in the system respectively.

the vapor phase of the component 1 that coexists with the liquid phase, is mixed with the component 2 that forms a pure gaseous phase. Molecules of the component 2 are diffusing into the liquid phase of the component 1 while molecules from the liquid phase of the component 1 are escaping into the gaseous phase. The system is not in a thermodynamic equilibrium. Although the temperature in the system is uniform, the pressure in the system is still changing over time due to the mass diffusion of the component 1 and 2. The same applies to the $\mathrm{SF}_{6}$ and He mixture. Here $\mathrm{SF}_{6}$ forms a liquid and a vapor phase and He remains in the gaseous phase. A mass diffusion of He into the liquid phase of $\mathrm{SF}_{6}$ would be indicated by a slow decrease in the pressure over time. Fig. 4.3 shows the temperature and the pressure in the convection apparatus over a time period of approximately $16 \mathrm{~d}$. The solid blue and the solid red line represent the temperature of the top and the bottom plate respectively. The solid green line shows the pressure in the system versus time. 


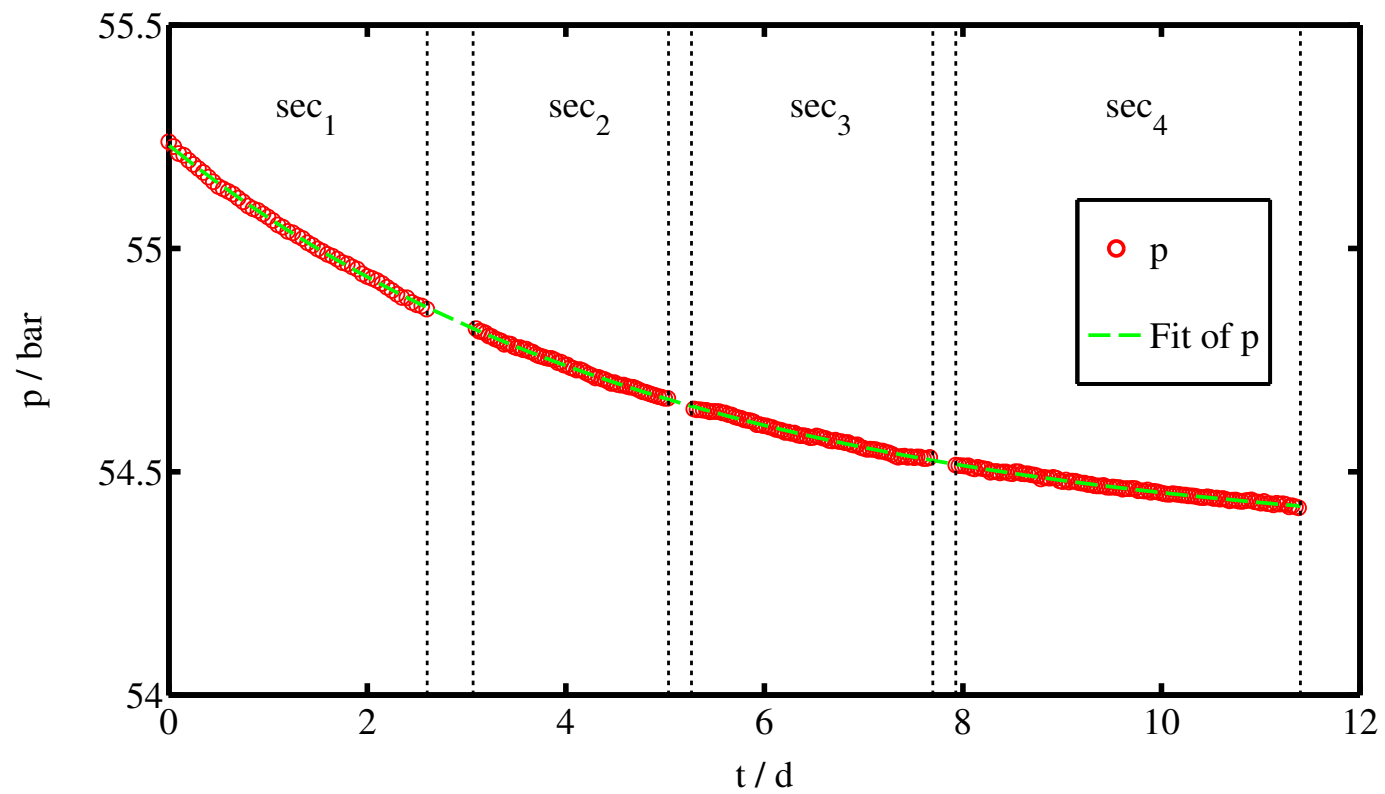

Figure 4.4: Offset correction of the pressure curve $p(t)$ in Fig. 4.3 over a time period of approximately $12 \mathrm{~d}$.

\begin{tabular}{lll}
\hline Parameter & Value & \\
\hline$\epsilon_{0}$ & 54.329 & bar \\
$\epsilon_{1}$ & 0.933 & bar \\
$\epsilon_{2}$ & $5.0543(2) \mathrm{d}$ \\
\hline
\end{tabular}

Table 4.4: Parameters for the fit of the pressure curve in Fig. 4.4.

When a new amount of $\mathrm{He}$ is filled into the convection cell, then the He gas mixes with the existing gaseous phase of $\mathrm{SF}_{6}$ and $\mathrm{He}$ in less than $1 \mathrm{~min}$ as shown in Section A.4. However, the concentration of $\mathrm{He}$ in the liquid phase of $\mathrm{SF}_{6}$ is still less than it would be in the concentration equilibrium. Thus $\mathrm{He}$ is diffusing into the liquid phase of $\mathrm{SF}_{6}$. This is why the pressure in Fig. 4.3 is still decreasing over time. In Fig. 4.3 He was filled into the convection cell at some instant in the past. Since the mass diffusion is much slower compared to the thermal diffusion, the time scales for the mass diffusion are much larger. In order to determine the time constant for the mass 
diffusion of He from the pressure curve $p(t)$ in Fig. 4.3 , the marked sections in Fig. 4.3 are first corrected for a pressure offset with section 2 as the reference pressure. The result of the offset corrected pressure curve is shown in Fig. 4.4. The open red circles are selected data points for the pressure in the system. The solid green line in Fig. 4.4 represents the fit of $p(t)$ with an exponential function of the type

$$
p(t)=\epsilon_{0}+\epsilon_{1} \exp \left(-\frac{t}{\epsilon_{2}}\right),
$$

by applying the method of non-linear least squares. The parameters of the fit are listed in Table 4.4. From the fit of the offset corrected pressure curve in Fig. 4.4. the time constant for the mass diffusion of He is found to be $\tau_{\mathrm{m}} \approx 5 \mathrm{~d}$. The waiting time $\tau_{\mathrm{m}_{\text {wait }}}$ until the pressure has stabilized is estimated to $\tau_{\mathrm{m}_{\text {wait }}} \approx 25 \mathrm{~d}$. If the time constant $\tau_{\mathrm{m}}$ is known, an estimation for the mass diffusion coefficient $D_{\mathrm{m}}$ for the diffusion of $\mathrm{He}$ into the liquid phase of $\mathrm{SF}_{6}$ can be made. For the diffusion in two dimensions [5], the two-dimensional mass diffusion coefficient $D_{\mathrm{m}}$ is defined as

$$
\left\langle x^{2}\right\rangle=2 D_{\mathrm{m}} t .
$$

The diffusion distance $x$ is replaced by the estimated height $H_{\text {lp }}$ of the total liquid phase of $\mathrm{SF}_{6}$ in the pressure vessel that is assumed to be $H_{\mathrm{lp}} \approx 5 \times 10^{-2} \mathrm{~m}$ and the time $t$ is replaced by the time constant $\tau_{\mathrm{m}}$ of the mass diffusion. By inserting the values for $H_{\mathrm{lp}}$ and $\tau_{\mathrm{m}}$ in Eq. (4.3), the mass diffusion coefficient $D_{\mathrm{m}}$ is estimated to

$$
\begin{aligned}
D_{\mathrm{m}} & =\frac{H_{\mathrm{lp}}^{2}}{2 \tau_{\mathrm{m}}} \\
& =2.9 \times 10^{-9} \mathrm{~m}^{2} \mathrm{~s}^{-1} .
\end{aligned}
$$




\begin{tabular}{lc}
\hline Thermophysical properties & Value \\
$\left(T=317.150 \mathrm{~K}, p\left(t_{0}\right)=55.239 \mathrm{bar}, p\left(t_{1}\right)=54.417 \mathrm{bar}\right)$ & \\
\hline${ }^{a}$ Vapor pressure $p_{1}(T)$ of $\mathrm{SF}_{6}$ & $36.272 \mathrm{bar}$ \\
Partial pressure $p_{2}\left(t_{0}, T\right)$ of He & $18.967 \mathrm{bar}$ \\
Partial pressure $p_{2}\left(t_{1}, T\right)$ of He & $18.145 \mathrm{bar}$ \\
a Density $\rho_{1}(T)$ of $\mathrm{SF}_{6}$ & $516.8 \quad \mathrm{~kg} \mathrm{~m}^{-3}$ \\
${ }^{a}$ Density $\rho_{2}\left(p_{2}\left(t_{0}\right), T\right)$ of $\mathrm{He}$ & $2.8567 \mathrm{~kg} \mathrm{~m}^{-3}$ \\
${ }^{a}$ Density $\rho_{2}\left(p_{2}\left(t_{1}\right), T\right)$ of $\mathrm{He}$ & $2.7339 \mathrm{~kg} \mathrm{~m}^{-3}$ \\
\hline
\end{tabular}

${ }^{a}$ Data taken from [52].

Table 4.5: Thermophysical properties of the gaseous phase of $\mathrm{SF}_{6}$ and He.

The result in Eq. (4.4) is of the same order of magnitude as the mass diffusion coefficient for He in water $D_{\mathrm{m}}=11.7 \times 10^{-9} \mathrm{~m}^{2} \mathrm{~s}^{-1}$ at $p=1.01325 \mathrm{bar}$ and $T=323.15 \mathrm{~K}$ that is found in the literature [74]. Although the exact height of the liquid phase of $\mathrm{SF}_{6}$ in the pressure vessel is not known, it is assumed that the pressure drop in Fig. 4.4 is caused by the diffusion of He into the liquid phase of $\mathrm{SF}_{6}$ which is located in the lower volume of the pressure vessel.

In order to get an impression of how much the concentration of He in the gaseous phase of $\mathrm{SF}_{6}$ and He has changed over a time period of approximately $12 \mathrm{~d}$, the concentration change of $\mathrm{He}$ in the gaseous phase at $t_{0}=0$ and at $t_{1}=11.4 \mathrm{~d}$ is estimated. The molar concentration of $\mathrm{He} y_{2}$ is calculated according to Eqs. A.1 and A.7b. By employing the thermophysical properties of $\mathrm{SF}_{6}$ and He in Table 4.5, the molar concentration of $\mathrm{He}$ in the gaseous phase is determined to

$$
\begin{aligned}
& y_{2}\left(t_{0}\right) \approx 16.8 \%, \\
& y_{2}\left(t_{1}\right) \approx 16.2 \% .
\end{aligned}
$$


The result in Eqs. (4.5a) and (4.5b) shows that the concentration of He in the gaseous phase of $\mathrm{SF}_{6}$ and He decreased by about $0.6 \%$ over a period of approximately $12 \mathrm{~d}$. Since a typical time interval for a measurement is less than $1 \mathrm{~h}$, the concentration of He is considered as being constant during this period. It is assumed that the thermal convection in the convection cell does not affect the diffusion process of He in the liquid phase of $\mathrm{SF}_{6}$ since most of the liquid $\mathrm{SF}_{6}$ is located below the bottom plate where no convection is present and where the fluid is supposed to be in a quiescent state.

\subsection{Droplet Formation}

This section describes the droplet formation that occurs at the top plate. During the experiments a liquid phase of $\mathrm{SF}_{6}$ and a gaseous phase of $\mathrm{SF}_{6}$ and $\mathrm{He}$ coexisted in the convection cell. When the bottom plate heater is switched on, the liquid phase of $\mathrm{SF}_{6}$ starts to evaporate and a film condensation sets in on the underside of the cold top plate. If the liquid film grows in thickness, the liquid-vapor interface becomes unstable and droplets start to form. If the droplets reached a critical size, they detach from the liquid film and fall back into the liquid pool above the bottom plate. Each time when a droplet falls down, a new droplet starts to develop at the same position as before. It is observed that the droplets align in a hexagonal close packed arrangement since this is the densest packing of spheres per unit area. Fig. 4.5 shows an image of the droplet pattern at $t \approx 27 \mathrm{~min}$ after the bottom plate heater is switched on.

A quantitative analysis of the droplet pattern is performed by determining the bond orientational order parameter $\phi_{N}$ which is a measure for the hexagonal symmetry of the droplet pattern and by determining the average droplet fall-down period $\tau_{\mathrm{fd}}$ 


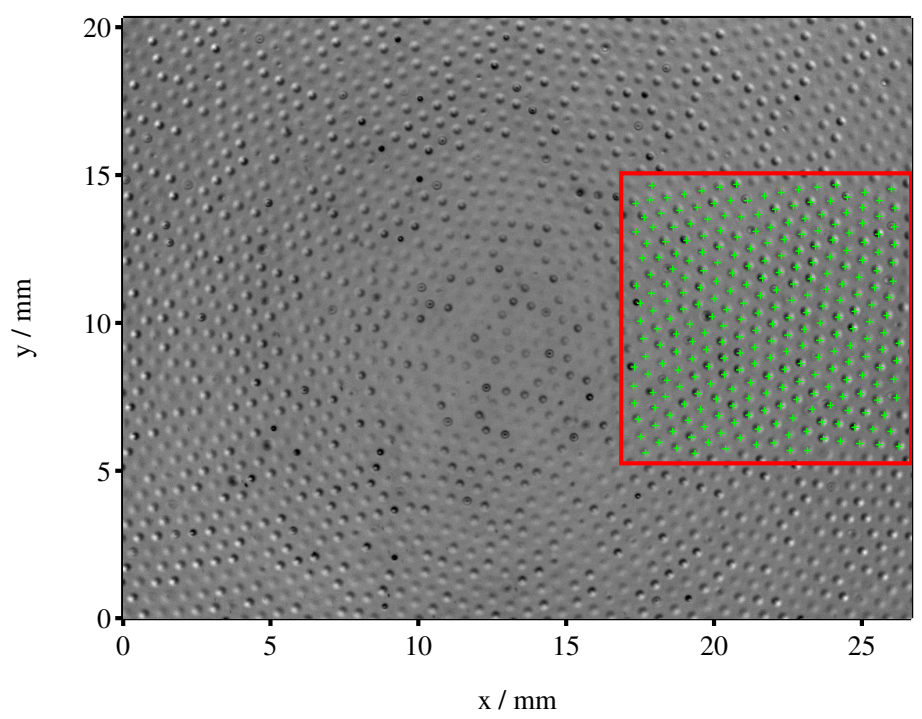

Figure 4.5: Image of the droplet pattern. The red rectangle marks the analyzed region $I_{\text {roi }}$ of the image. The green crosses mark the center of each droplet within $I_{\text {roi }}$.

which determines if the condensation on the underside of the top plate can be considered as being stable or not. In order to determine the bond orientational order parameter, the center of each droplet in a certain image region must be identified. The average droplet fall-down period requires that the tracks of individual droplets in an image sequence are detected. Section 4.2.1 describes the image processing techniques that were applied in order to identify the center and the tracks of individual droplets. The parameters $\phi_{N}$ and $\tau_{\mathrm{fd}}$ are analyzed in order to show that a mass flux is mandatory for maintaining the hexagonal symmetry of the droplet pattern. In order to show this, two distinct scenarios are investigated. The first scenario in Section 4.2 .2 describes the situation when the bottom plate heater is switched off and the droplet pattern relaxes. The second scenario in Section 4.2.2 describes the situation when the bottom plate heater is switched on and the droplet pattern starts to build up. In order to analyze the temporal evolution of $\phi_{N}$ and $\tau_{\mathrm{fd}}$, for both scenarios an image sequence of $n=5000$ images with a frame rate of $5 \mathrm{fps}$ is recorded. Each image sequence lasts about $17 \mathrm{~min}$. 
The images of the droplet pattern can only be analyzed if the cloud-like patterns in Section 4.3 are not visible since the dark patterns cover most of the droplets. For the scenario when the bottom plate heater is switched off, it takes about $t \approx 10 \mathrm{~min}$ until the cloud-like patterns vanished. The image sequence can first be taken when the cloud-like patterns disappeared. The scenario when the bottom plate heater is switched off is shown in Fig. 4.2. The black dotted line and the horizontal one-sided black arrow mark the point until the cloud-like patterns are visible. The vertical red dotted lines and the horizontal two-sided red arrow mark the time interval when the image sequence is recorded. For the scenario when the bottom plate heater is switched on, it takes about $t \approx 30 \mathrm{~min}$ until the cloud-like patterns do appear. Therefore the image sequence must be taken within this time interval. The scenario when the bottom plate heater is switched on is shown in Fig. 4.1. The black dotted line and the horizontal one-sided black arrow mark the point when the cloud-like patterns begin to appear. The vertical red dotted lines and the horizontal two-sided red arrow mark the time interval when the image sequence is recorded. A discussion of the results and a theoretical explanation for the droplet formation is presented in Section 4.2.4.

\subsubsection{Image analysis}

\section{Determination of the center of a droplet}

Fig. 4.5 shows an image of the droplet pattern. The red rectangle marks the image region $I_{\text {roi }}$ which is analyzed to determine $\phi_{N}$ and $\tau_{\mathrm{fd}}$. In order to identify the center of each droplet within $I_{\text {roi, }}$ all images are first background corrected in order to remove inhomogeneities in the intensity profile which are caused by the method of illumination. To perform a background correction, each image is divided by a two- 


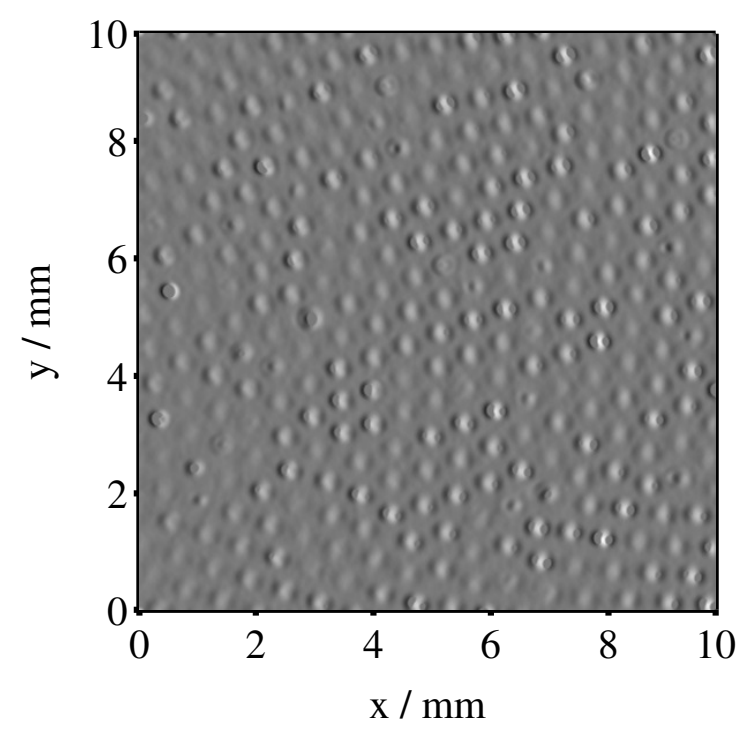

(a)

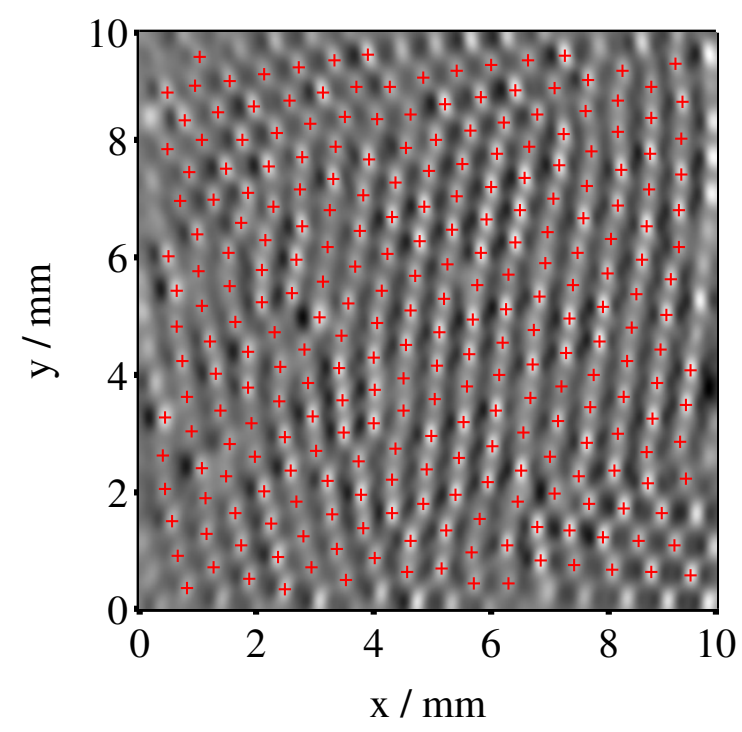

(b)

Figure 4.6: (a) shows the gradient map $I_{\mathrm{gx}}(x, y)$ in $x$-direction of the image region $I_{\text {roi }}$ in Fig. 4.5. (b) shows the convolution of $I_{\mathrm{gx}}(x, y)$ with a sample function $H(x, y)$.

dimensional Gaussian intensity profile. This results in the two-dimensional normalized intensity profile $I_{\mathrm{n}}(x, y)$ which is defined as follows

$$
I_{\mathrm{n}}(x, y)=1-\frac{I\left(x_{i}, y_{j}\right)}{I_{0}\left(x_{i}, y_{j}\right)}
$$

The indexes $i=1, \ldots, N$ and $j=1, \ldots, M$ in Eq. (4.6) label the number of rows and columns of the analyzed image region respectively. After the background correction is performed, all images are scaled with the same scaling factor to a 16-bit grayscale image. In order to find the center of each droplet in $I_{\text {roi }}$, the gradient map

$$
I_{\mathrm{gx}}(x, y)=\nabla_{x} I_{\mathrm{n}}(x, y)
$$

of the image in $x$-direction is determined as shown in Fig. 4.6a. The droplets also exhibit an inhomogeneous intensity profile. A droplet in the image region $I_{\text {roi }}$ features 
a region of higher and lower intensity. The intensity profile of a droplet in $I_{\text {roi }}$ corresponds to the profile of the first derivative of a two-dimensional Gaussian. Therefore the gradient map in Eq. [4.7) is convolved [4, 26] with a sample function $H(x, y)$ as follows

$$
I_{\mathrm{cv}}(x, y)=\left(I_{\mathrm{gx}} \star H\right)(x, y)=\sum_{x^{\prime}=N / 2}^{N-1 / 2} \sum_{y^{\prime}=M / 2}^{M-1 / 2} I_{\mathrm{gx}}\left(x^{\prime}, y^{\prime}\right) H\left(x-x^{\prime}, y-y^{\prime}\right) .
$$

$H(x, y)$ in Eq. (4.8) corresponds to the first derivative of a two-dimensional Gaussian. The dimensions of $H(x, y)$ are the same as for a droplet in the gradient map. The convolution with a sample function is shown in Fig. 4.6b and results in a high correlation value if a droplet is encountered in the image region $I_{\text {roi }}$. A peak-finding algorithm [51] is applied to find the peaks with the highest correlation value in Fig. $4.6 \mathrm{~b}$. The peaks with a high correlation value correspond to the center of a droplet. In Fig. $4.6 \mathrm{~b}$ the center of each droplet is marked by a red cross.

\section{Determination of the bond orientational order parameter $\phi_{N}$}

In order to verify that a mass flux is mandatory for maintaining the hexagonal symmetry of the droplet pattern, the bond orientational order parameter $\phi_{N}$ is determined which is a measure for the symmetry of a lattice [7, 65]. $\phi_{N}$ is defined as

$$
\phi_{N}=\frac{1}{N}\left|\sum_{j=1}^{N} \exp \left(i n \theta_{j}\right)\right| .
$$

The idea is to perform a quantitative analysis of the droplet pattern when the bottom plate heater is switched on and off. Since the mass flux stops when the heater is switched off, the droplet pattern relaxes and this should be noticeable in a decrease of $\phi_{N}$ over time. On the other hand when the heater is switched on, the mass flux sets 
in and the droplet pattern builds up. This should be noticeable in an increase of $\phi_{N}$ over time. Since the droplets form a hexagonal lattice, the number of nearest neighbors equals $N=6$. An ideal hexagonal lattice would yield in a value of $\phi_{6}=1$ [7]. In order to determine $\phi_{6}$, the $N=6$ nearest neighbors of each droplet in the image region $I_{\text {roi }}$ must be identified. In the next step, the relative coordinates of the nearest neighbors with respect to the central droplet are determined. The Cartesian coordinates are then converted into polar coordinates. Next the angle $\theta_{j},(j=1, \ldots, 6)$ between two consecutive neighbors with the central droplet as the apex is determined. $\phi_{6}$ is calculated by inserting $\theta_{j}$ into Eq. (4.9) and by taking the average of $\phi_{6}$ for all droplets in the image region $I_{\text {roi }}$.

\section{Determination of the average droplet fall-down period $\tau_{\mathrm{fd}}$}

The droplet fall-down period is the time between two consecutive fall-down events. In order to determine the average droplet fall-down period $\tau_{\mathrm{fd}}$, the tracks of individual droplets in $I_{\text {roi }}$ are identified by applying a particle tracking algorithm [9]. The total image sequence of $n=5000$ images is divided into subsequences of $n=500$ images. For each subsequence the tracks of individual droplets are identified. The normalized standard deviation of the intensity profile of a droplet is a good measure to identify when a droplet detaches from the top plate and falls down. For each identified droplet track in the image subsequence, the normalized standard deviation over time is autocorrelated and the first and the second maximum of the autocorrelation curve is detected. The difference between the first and the second maximum corresponds to the fall-down period of a droplet. Next the average droplet fall-down period $\tau_{\mathrm{fd}}$ is determined by taking the average of the fall-down periods of all droplets in the image subsequence. This results in one value for the average droplet fall-down period for each image subsequence. 


\section{Determination of the mean distance $d_{n n}$ between two neighboring droplets}

Besides the bond orientational order parameter $\phi_{N}$ and the average droplet falldown period $\tau_{\mathrm{fd}}$, the mean distance $\mathrm{d}_{\mathrm{nn}}$ between two neighboring droplets in Fig. 4.5 is determined. Therefore the discrete Fourier transform (DFT)

$$
F\left(k_{\mathrm{x}}, k_{\mathrm{y}}\right)=\sum_{x=0}^{N-1} \sum_{y=0}^{M-1} f(x, y) e^{-i 2 \pi\left(\frac{k_{x} x}{N}+\frac{k_{\mathrm{y}} y}{M}\right)}
$$

is employed as defined in [4, 26]. The DFT is applied to the background corrected image in Fig. 4.5 in order to determine the distribution of $k_{\mathrm{x}}$ and $k_{\mathrm{y}}$ in the frequency domain. Next the logarithmic power spectral density (LPSD) $G\left(k_{x}, k_{\mathrm{y}}\right)$ is calculated as follows

$$
G\left(k_{\mathrm{x}}, k_{\mathrm{y}}\right)=\log _{10}\left(\left|F\left(k_{\mathrm{x}}, k_{\mathrm{y}}\right)\right|^{2}\right)
$$

Finally $G\left(k_{\mathrm{x}}, k_{\mathrm{y}}\right)$ is transformed into $G(r, \phi)$ and the one-dimensional LPSD

$$
G\left(r^{\prime}\right)=\int_{0}^{2 \pi} \int_{r^{\prime}}^{r^{\prime}+\Delta r} G(r, \phi) r \mathrm{~d} \phi \mathrm{d} r
$$

is determined by azimuthally integrating $G(r, \phi)$. The frequency that corresponds

to the second largest maximum in $G\left(r^{\prime}\right)$ is used to calculate the mean distance $d_{n n}$ between two neighboring droplets in Fig. 4.5 .

\subsubsection{Heater switched off}

As already mentioned in the beginning of this section, the image sequence for the determination of $\phi_{6}$ is taken in the time interval that is marked by the horizontal two-sided red arrow in Fig. 4.2. The bottom plate heater is switched off at $t_{0}=0$. The recording of the image sequence starts at $t_{1} \approx 15 \mathrm{~min}$ after the heater is switched off. The images are analyzed as described in Section 4.2.1 and the bond orienta- 


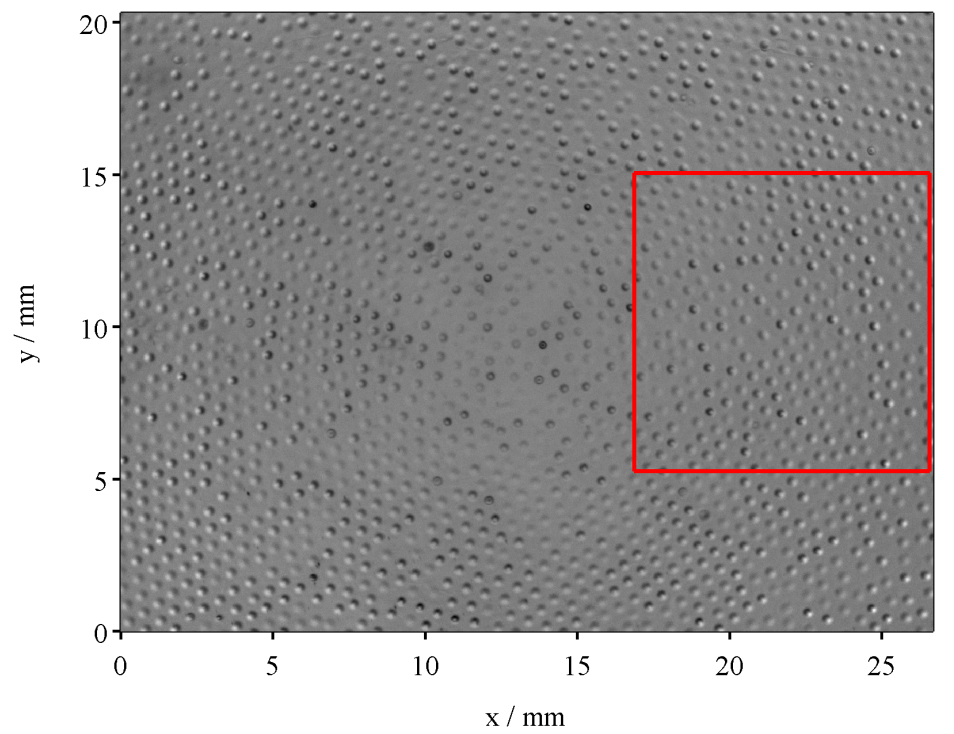

(a)

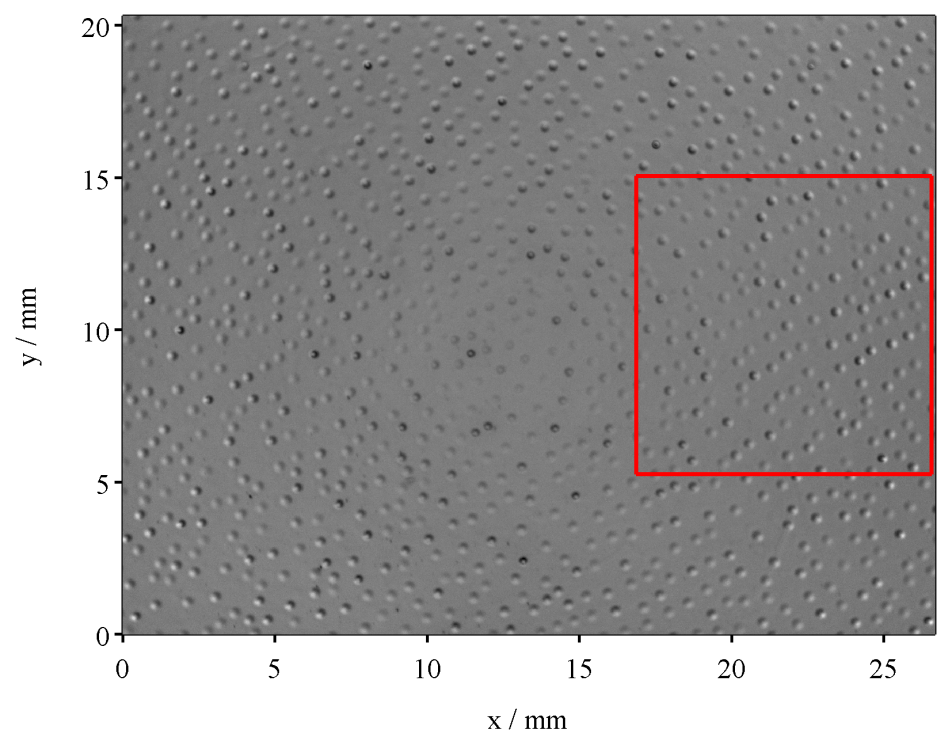

(b)

Figure 4.7: The bottom plate heater is switched off at $t_{0}=0$. (a) and (b) show the droplet pattern at $t_{1} \approx 15 \mathrm{~min}$ and $t_{2} \approx 32 \mathrm{~min}$ respectively. 


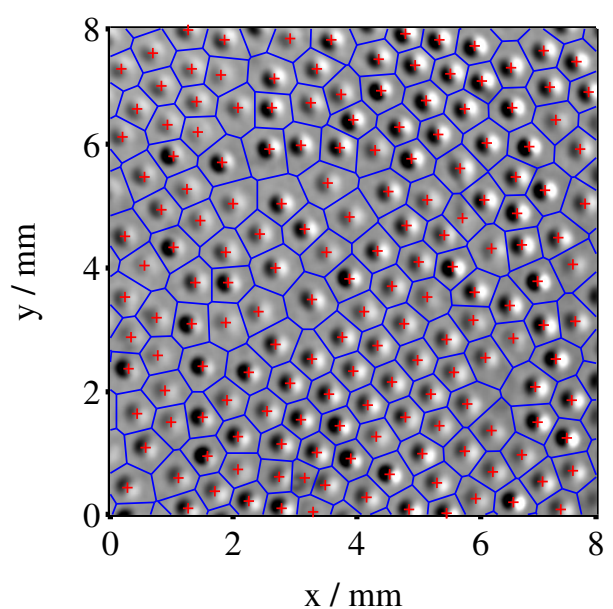

(a)

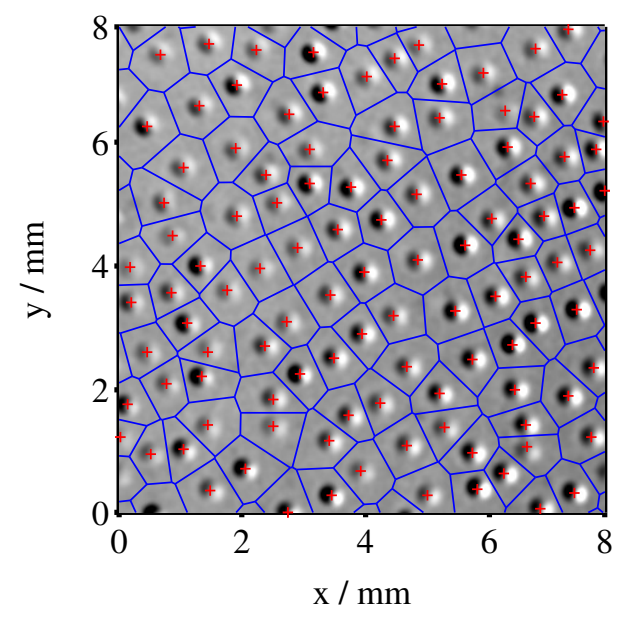

(b)

Figure 4.8: (a) and (b) show the Voronoi map of $I_{\text {roi }}$ in Fig. 4.7a and in Fig. 4.7b respectively.

tional order parameter $\phi_{6}$, the fall-down period $\tau_{\mathrm{fd}}$ and the mean distance $\mathrm{d}_{\mathrm{nn}}$ are determined. Fig. $4.7 \mathrm{a}$ and Fig. $4.7 \mathrm{~b}$ show the first and the last image of the image sequence. In Fig. $4.7 \mathrm{~b}$ it is already visible that the droplet density per unit area has decreased and the hexagonal symmetry of the droplet pattern disappears. $\phi_{6}$ of the image region $I_{\text {roi }}$ in Fig. $4.7 \mathrm{a}$ and in Fig. $4.7 \mathrm{~b}$ is determined to

$$
\begin{aligned}
& \phi_{6}\left(t_{1}\right)=0.58, \\
& \phi_{6}\left(t_{2}\right)=0.33 .
\end{aligned}
$$

The value of $\phi_{6}$ at $t_{2}$ is much smaller than at $t_{1}$. This shows that the hexagonal symmetry of the droplet pattern declines when the heater is switched off and the mass flux stops. For comparison if $t \ll 0$, then the condensation can be considered as being stable and the bond orientational order parameter is determined to

$$
\phi_{6}(t \ll 0)=0.90
$$




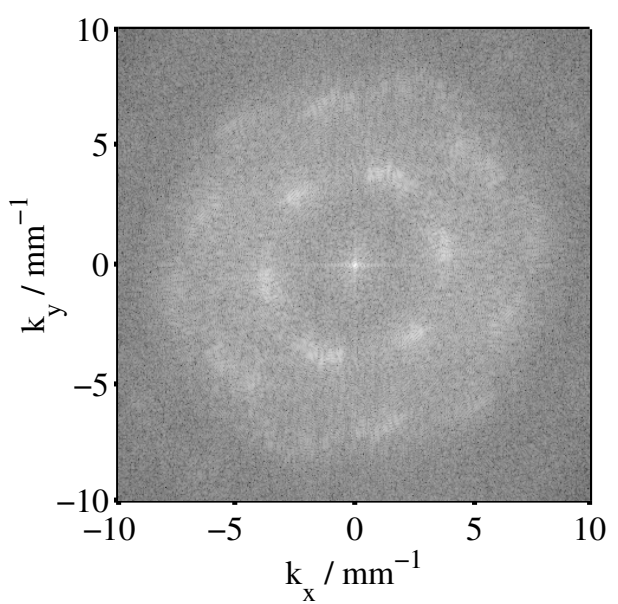

(a1)

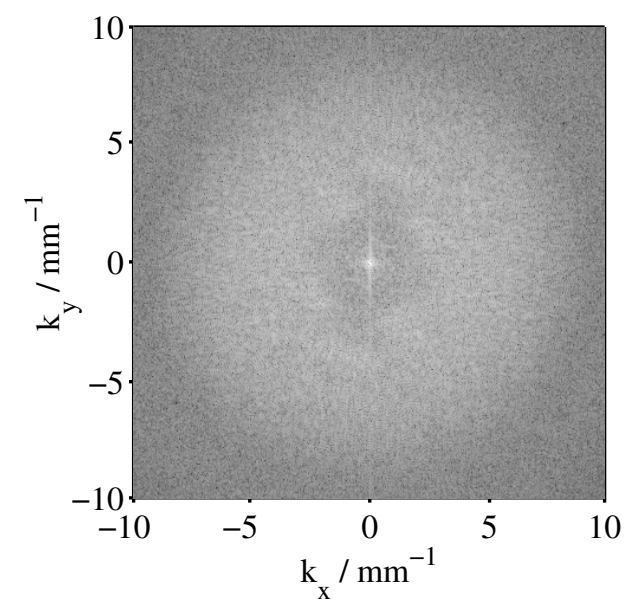

(b1)

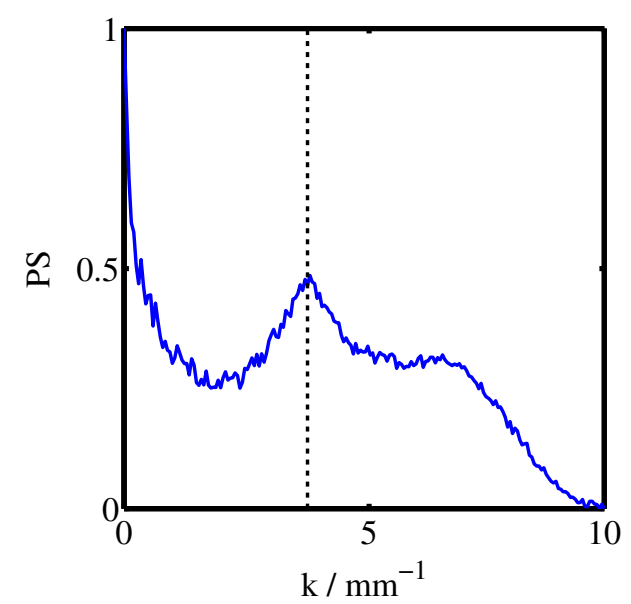

(a2)

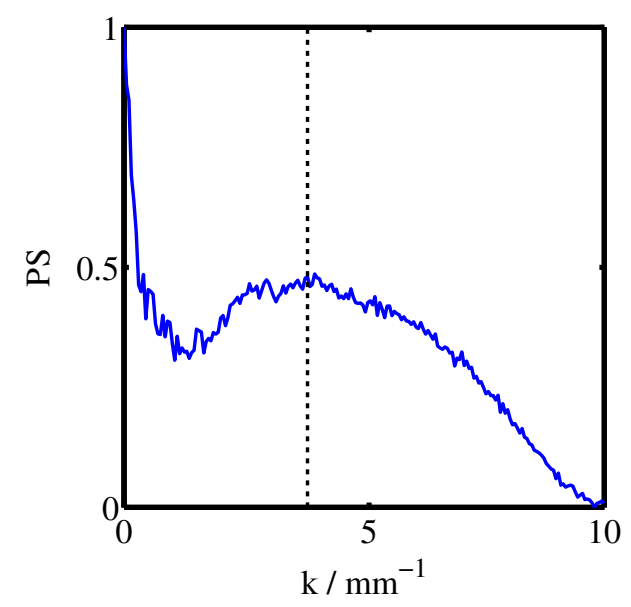

(b2)

Figure 4.9: (a1) and (b1) show the two-dimensional LPSD of Fig. 4.7a and Fig. 4.7b respectively. (a2) and (b2) show the one-dimensional azimuthally averaged LPSD of (a1) and (b1) respectively.

The decline of the hexagonal symmetry can also be identified by performing a Voronoi tessellation. Fig. $4.8 \mathrm{a}$ and Fig. $4.8 \mathrm{~b}$ show the Voronoi map of the image region $I_{\text {roi }}$ in Fig. $4.7 \mathrm{a}$ and in Fig. $4.7 \mathrm{~b}$ respectively. In an ideal hexagonal lattice all Voronoi cells would be regular and equilateral hexagons and the distance $d_{n n}$ between two neighboring droplets would always be the same. In Fig. $4.8 \mathrm{~b}$ it can be seen that the hexagonal symmetry of the droplet pattern is completely declined. 
Most of the droplets have less or more than $N=6$ nearest neighbors which can be identified by counting the number of edges of a Voronoi cell. Also the length of the edges of the Voronoi cells are not equal which means the mean distance between two neighboring droplets is not equal any more.

In order to determine the mean distance $d_{n n}$ between two neighboring droplets, the two-dimensional and the one-dimensional LPSD of Fig. 4.7a and Fig. $4.7 \mathrm{~b}$ is determined as described in Section 4.2.1. In Fig. 4.9a1 the hexagonal symmetry of the droplet pattern is still visible while in Fig. $4.9 \mathrm{~b} 1$ the droplets are randomly oriented and the $\mathbf{k}$ vectors become uniformly distributed in the frequency domain. Fig. $4.9 \mathrm{a} 2$ and Fig. $4.9 \mathrm{~b} 2$ show the frequency distribution $|\mathbf{k}|$. The dotted line in Fig. $4.9 \mathrm{a} 2$ and in Fig. 4.9b2 marks the mean distance $\mathrm{d}_{\mathrm{nn}}$ in the frequency domain. From the wave number that corresponds to the second largest peak, the mean distance is determined to

$$
\begin{aligned}
& d_{\mathrm{nn}}\left(t_{1}\right)=0.52 \mathrm{~mm}, \\
& d_{\mathrm{nn}}\left(t_{2}\right)=0.52 \mathrm{~mm} .
\end{aligned}
$$

Although $\phi_{6}$ is clearly decreasing between $t_{1}$ and $t_{2}$, the mean distance $d_{n n}$ at $t_{2}$ is still the same as for $t_{1}$ since the position of the peak in Fig. $4.9 \mathrm{a} 2$ and in Fig. $4.9 \mathrm{~b} 2$ did not change. However, in Fig. $4.9 \mathrm{~b} 2$ it can be seen that the second largest peak is broadened and not clearly visible any more which means the distances between two neighboring droplets are now distributed over a wide range since the hexagonal symmetry is declined. So the parameter $\phi_{6}$ seems to be better suited as a measure for the hexagonal symmetry of the droplet pattern.

Fig. $4.10 \mathrm{a}$ shows the decline of $\phi_{6}$ over time when the heater is switched off. In Fig. $4.10 \mathrm{~b}$ the average droplet fall-down period $\tau_{\mathrm{fd}}$ is determined for each image 


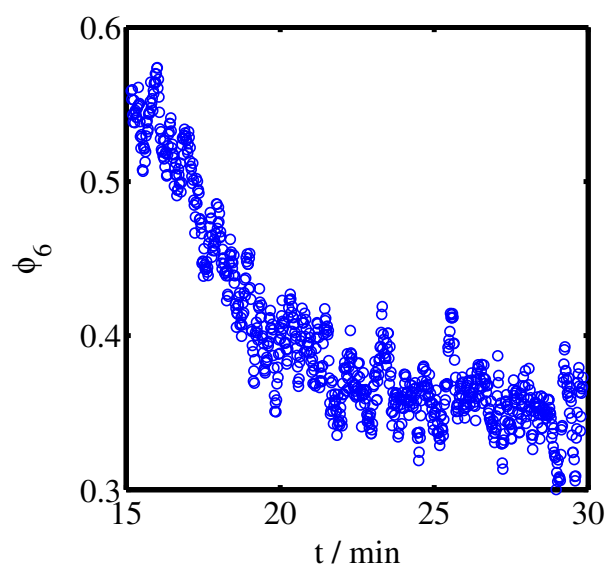

(a)

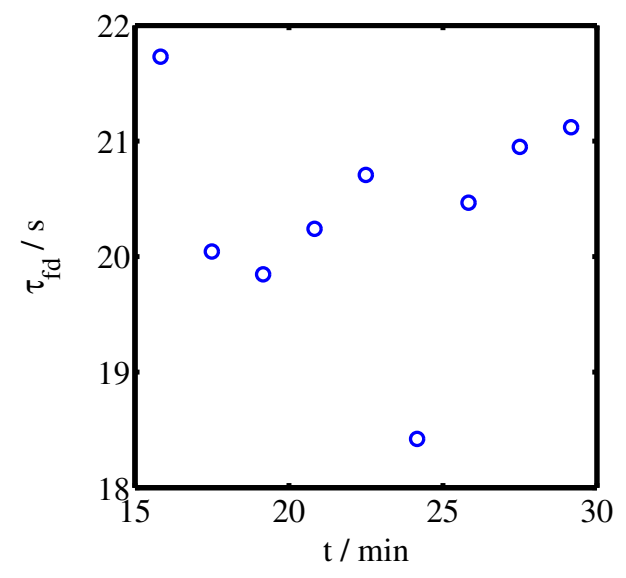

(b)

Figure 4.10: (a) shows the evolution of $\phi_{6}$ over time. (b) shows the average droplet fall-down period for each image subsequence.

subsequence as described in Section 4.2.1. $\tau_{\mathrm{fd}}$ neither increases nor decreases but fluctuates around a mean value while $\phi_{6}$ is declining at the same time. This can be related to the fact that the droplet density in the image region $I_{\text {roi }}$ is decreasing over time but the fall-down period of the still existing droplets remains the same since there is still some condensation on the underside of the top plate. For comparison if $t \ll 0$, then the average droplet fall-down period and the mean distance $\mathrm{d}_{\mathrm{nn}}$ are found to

$$
\tau_{\mathrm{fd}}(t \ll 0) \approx 5 \mathrm{~s}
$$

and

$$
d_{\mathrm{nn}}=0.51 \mathrm{~mm}
$$

respectively. $\tau_{\mathrm{fd}}$ increases for $t>0$ since the mass flux from the surface of the liquid phase of $\mathrm{SF}_{6}$ to the top plate stops. However, it takes more than $1 \mathrm{~h}$ until the bottom plate reached the temperature equilibrium. Since the liquid phase of $\mathrm{SF}_{6}$ is still warmer than the top plate, it is assumed that there is still a weak evaporation and a condensation at the top plate which continues until the liquid phase of $\mathrm{SF}_{6}$ has com- 
pletely cooled down. It is conjectured that $\tau_{\mathrm{fd}}$ increases further for $t \gg 0$ since some droplets which still remain on the underside of the top plate can persist for hours or several days until they eventually fall down. For $t \ll 0$, the mean distance $d_{\text {nn }}$ between two neighboring droplets is slightly smaller compared to the transient state when the heater is switched off or on. It is conjectured that the droplet pattern still evolves if the system is in a transient state and that the droplets are getting closer arranged.

\subsubsection{Heater switched on}

The image sequence for the determination of $\phi_{6}$ is taken in the time interval that is marked by the horizontal two-sided red arrow in Fig. 4.1. The bottom plate heater is switched on at $t_{0}=0$. The recording of the image sequence starts at $t_{1} \approx 10 \mathrm{~min}$ after the heater is switched on. The images are analyzed as described in Section 4.2.1 and the bond orientational order parameter $\phi_{6}$, the fall-down period $\tau_{\mathrm{fd}}$ and the mean distance $d_{n n}$ are determined. Fig. 4.11a and Fig. 4.11b show the first and the last image of the image sequence. In Fig. 4.11a it can be seen that the hexagonal droplet pattern is nearly fully developed. It seems as if the droplet pattern builds up immediately after the bottom plate heater is switched on and the condensation sets in. $\phi_{6}$ of the image region $I_{\text {roi }}$ in Fig. 4.11a and in Fig. $4.11 \mathrm{~b}$ is determined to

$$
\begin{aligned}
& \phi_{6}\left(t_{1}\right)=0.80, \\
& \phi_{6}\left(t_{2}\right)=0.87 .
\end{aligned}
$$

$\phi_{6}$ increases only slightly between $t_{1}$ and $t_{2}$ which shows that the hexagonal symmetry of the droplet pattern is almost completely developed at $t_{1} \approx 10 \mathrm{~min}$ after the heater is switched on. As already mentioned in Section 4.2.2, if $t \ll 0$, then the value 


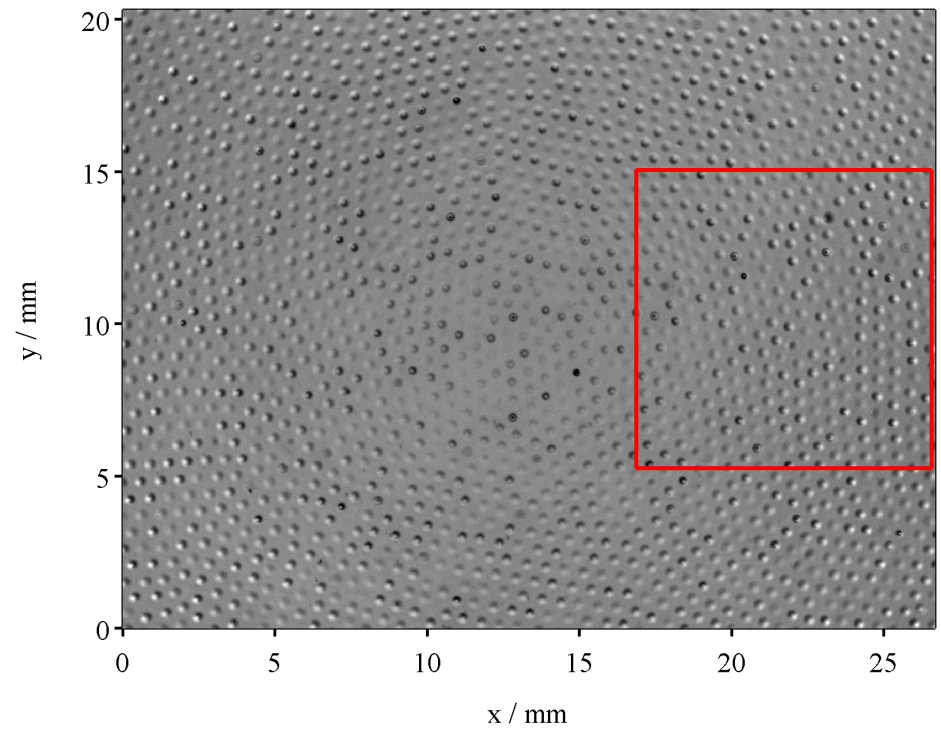

(a)

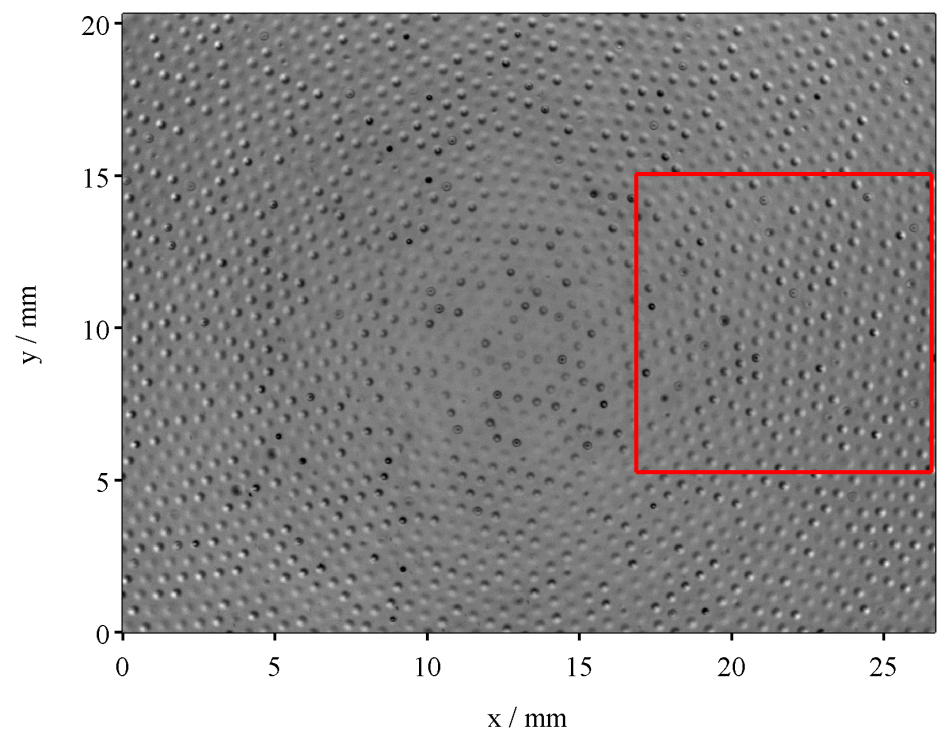

(b)

Figure 4.11: The bottom plate heater is switched on at $t_{0}=0$. (a) and (b) show the droplet pattern at $t_{1} \approx 10 \mathrm{~min}$ and at $t_{2} \approx 27 \mathrm{~min}$ respectively. 


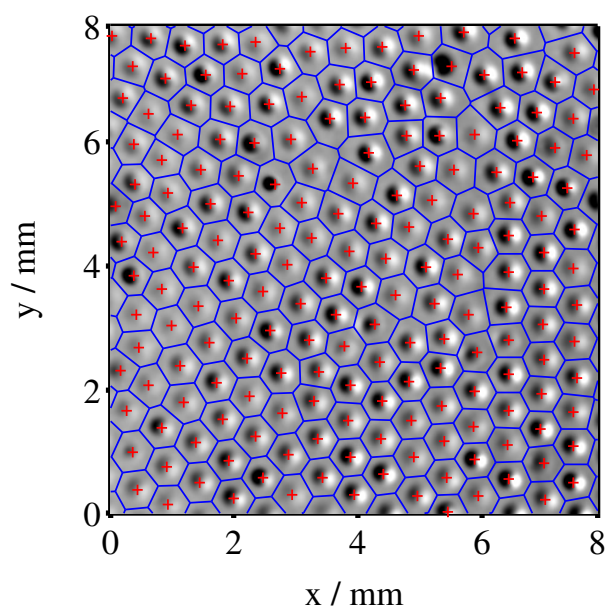

(a)

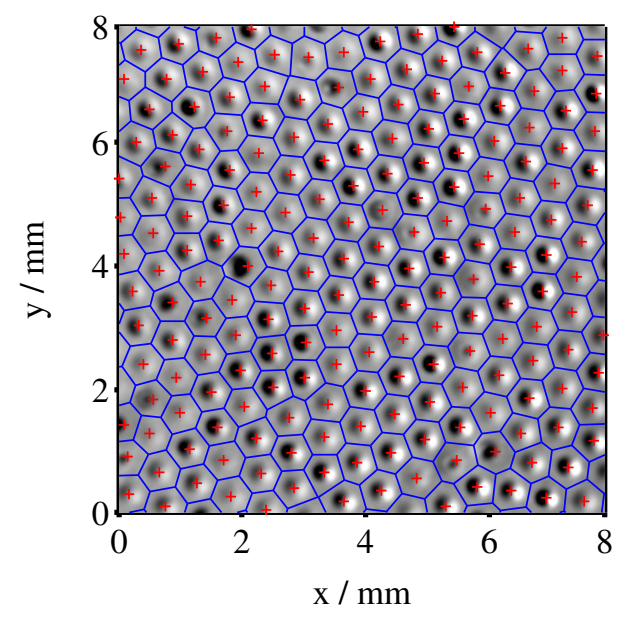

(b)

Figure 4.12: (a) and (b) show the Voronoi map of $I_{\text {roi }}$ in Fig. 4.11a and in Fig. 4.11b respectively.

of $\phi_{6}$ is found to be $\phi_{6}=0.90$. So at $t_{2} \approx 27 \mathrm{~min}$, the condensation at the top plate can be considered as being almost stable. The evolution of the droplet pattern seems to correlate with the temperature of the bottom plate $T_{\mathrm{b}}$ in Fig. 4.1 since the bottom plate has already reached the setpoint temperature after $t_{1} \approx 10 \mathrm{~min}$.

In Fig. 4.12a and in Fig. 4.12b a Voronoi tessellation of the image region $I_{\text {roi }}$ in Fig. $4.11 \mathrm{a}$ and in Fig. $4.11 \mathrm{~b}$ is performed. The Voronoi map indicates the increase of the hexagonal symmetry of the droplet pattern. After $t_{1} \approx 10 \mathrm{~min}$ only a few Voronoi cells exist that have $N \neq 6$ nearest neighbors. Most of the cells have $N=6$ nearest neighbors although the edges of the hexagons are not exactly equilateral. After $t_{2} \approx 27$ min nearly all cells have $N=6$ nearest neighbors and the edges of the cells are almost equilateral. But still there exist some defect locations where droplets have for instance $N=5$ nearest neighbors. Since the Voronoi cells are nearly ideal hexagons in Fig. $4.12 \mathrm{~b}$, this can also be seen in Fig. $4.13 \mathrm{~b} 2$ where the second largest peak is now clearly pronounced and much narrower compared to the peaks in Fig. 4.9 . The mean distance $d_{n n}$ between two neighboring droplets is determined as described 


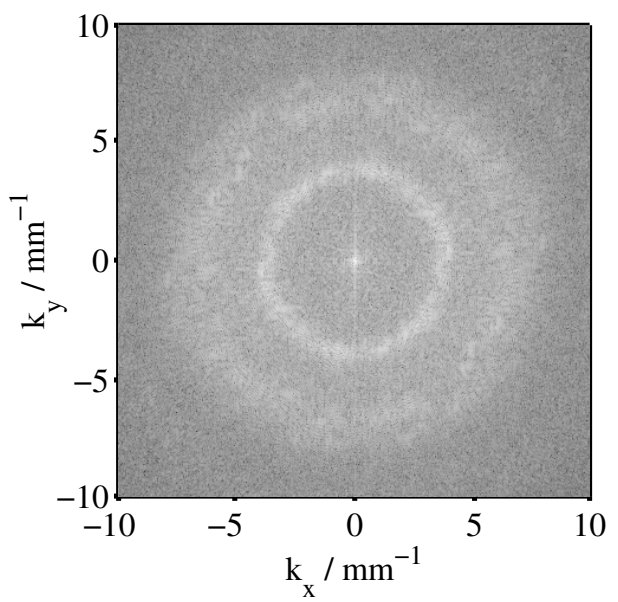

(a1)

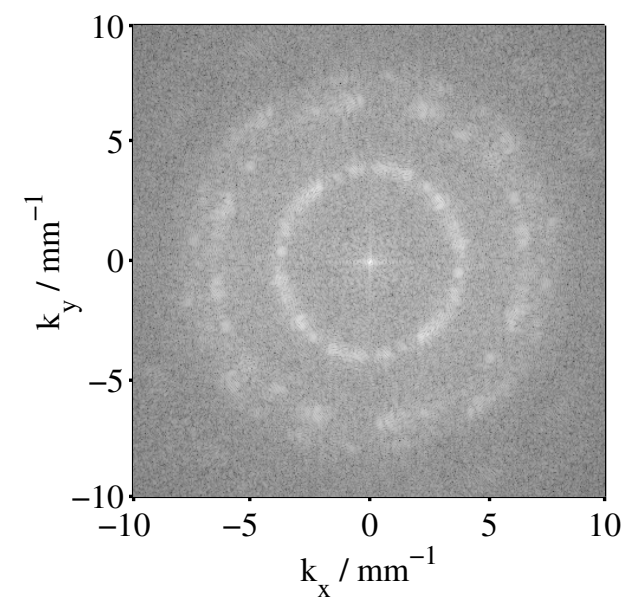

(b1)

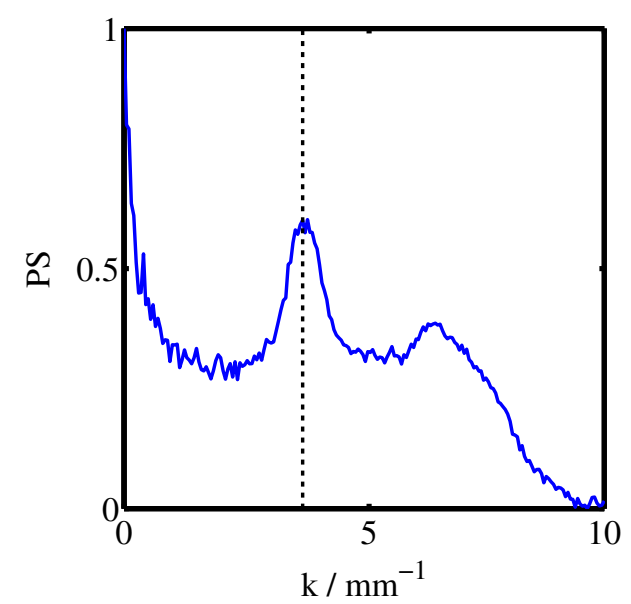

(a2)

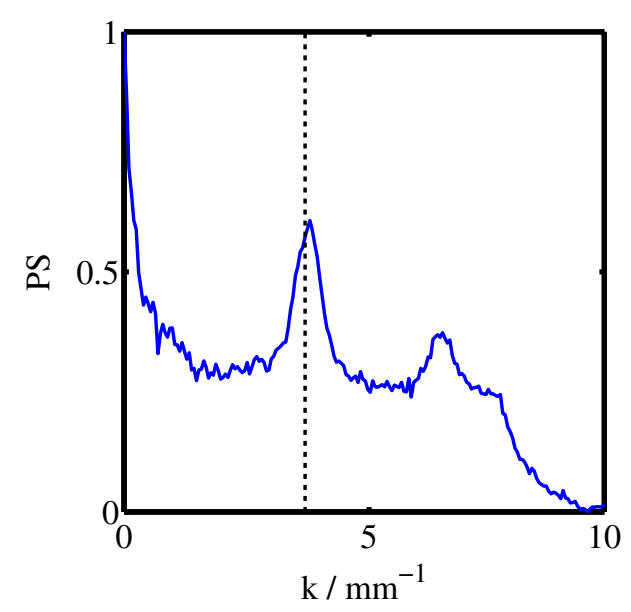

(b2)

Figure 4.13: (a1) and (b1) show the two-dimensional LPSD of Fig. 4.11a and Fig. $4.11 \mathrm{~b}$ respectively. (a2) and (b2) show the one-dimensional azimuthally averaged LPSD of (a1) and (b1) respectively.

in Section 4.2.1. The frequencies which correspond to the second largest peak in Fig. $4.13 \mathrm{a} 2$ and in Fig. 4.13b2 are narrowly distributed compared to the frequencies in Fig. 4.9 since the hexagonal symmetry is now fully developed and the droplets are arranged almost equidistant to each other. However, the $\mathbf{k}$ vectors are still uniformly distributed in the frequency domain as can be seen in Fig. 4.13a1. In Fig. 4.13b1 the hexagonal symmetry becomes visible in the frequency domain and the peak in Fig. 


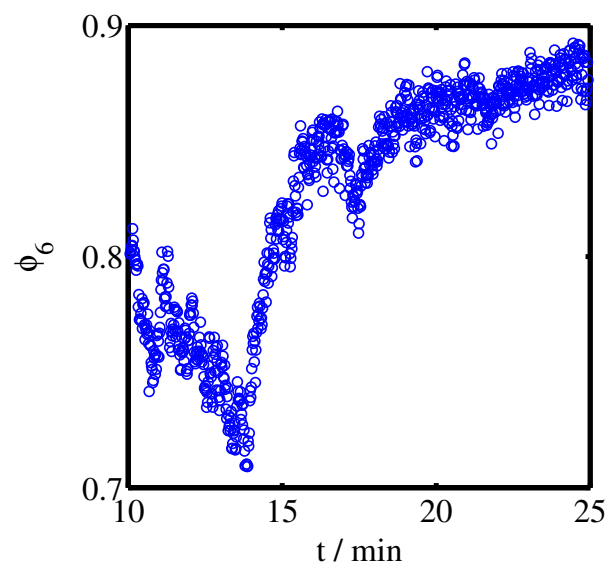

(a)

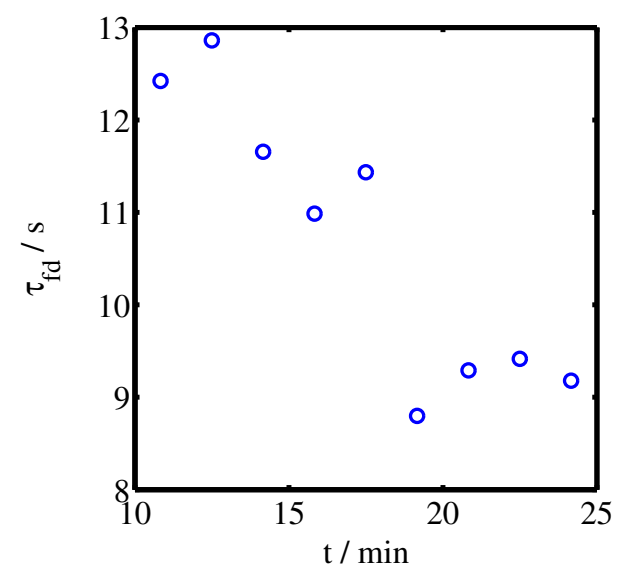

(b)

Figure 4.14: (a) shows the evolution of $\phi_{6}$ over time. (b) shows the average droplet fall-down period for each image subsequence.

$4.13 \mathrm{~b} 2$ is further narrowing. From the wave number marked by the dotted line in Fig. 4.13a2 and in Fig. 4.13b2, the mean distance $\mathrm{d}_{\mathrm{nn}}$ is calculated to

$$
\begin{aligned}
& d_{\mathrm{nn}}\left(t_{1}\right)=0.54 \mathrm{~mm}, \\
& d_{\mathrm{nn}}\left(t_{2}\right)=0.53 \mathrm{~mm} .
\end{aligned}
$$

The mean distance $d_{n n}$ at $t_{1}$ and $t_{2}$ is almost the same since the position of the peak in Fig. 4.13a2 and in Fig. 4.13b2 did not change. Again it is evident that $\phi_{6}$ is better suited in order to quantify the hexagonal symmetry of the droplet pattern.

Fig. $4.14 \mathrm{a}$ shows that $\phi_{6}$ is still increasing over time between $t_{1}$ and $t_{2}$ which means the droplet pattern is not yet fully developed. Fig. $4.14 \mathrm{~b}$ shows the average droplet fall-down period for each image subsequence. $\tau_{\mathrm{fd}}$ still decreases over time which shows that the condensation is not stable yet. It is supposed that the liquid phase of $\mathrm{SF}_{6}$ is still heating up and did not yet reach the temperature of the bottom plate. Thus the condensation rate at the top plate is still increasing what results in a decrease of the average droplet fall-down period. It can be concluded that the bond orientational 
order parameter $\phi_{6}$ is suited as a measure for the hexagonal symmetry of the droplet pattern. The decrease and increase of $\phi_{6}$ over time indicates that a constant mass flux and a stable condensation are essential for maintaining the hexagonal symmetry of the droplet pattern.

\subsubsection{Discussion}

As already mentioned in the beginning of this section, the evaporation results in a mass flux from the surface of the liquid phase of $\mathrm{SF}_{6}$ to the top plate and causes a film condensation on the underside of the top plate. The liquid film grows in thickness and eventually the interface between the liquid film and the subjacent gaseous phase becomes Rayleigh-Taylor unstable as described in Section 2.5.2. The instability results in the formation of a corrugated surface as illustrated in Fig. 4.15. The condensation occurs in the crests and the fluid flows into the sinks where the droplets start to form. The droplets align at a distance $\lambda$ that equals the mean distance $d_{n n}$ between two neighboring droplets. If a droplet reached a critical size, it detaches and falls back into the liquid pool above the bottom plate. The critical wavelength $\lambda_{\mathrm{c}}$ is estimated according to Eq. (2.36) in order to verify if $\lambda_{\mathrm{c}}$ compares to the mean distance $d_{n n}$ in Eq. (4.17) for the case when the condensation at the top plate is stable. In order to calculate $\lambda_{c}$, it is assumed that the liquid film at the top plate is composed of pure $\mathrm{SF}_{6}$. The subjacent gaseous phase is considered to be a mixture of $\mathrm{SF}_{6}$ and He. By employing the thermophysical properties in Table 4.6, the critical wavelength is determined to

$$
\lambda_{\mathrm{c}}=5.2 \times 10^{-4} \mathrm{~m} .
$$




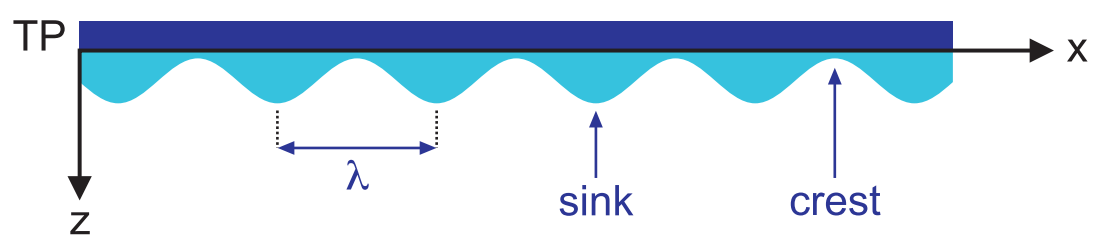

Figure 4.15: Schematic drawing of the droplet formation on the underside of the top plate. $\lambda$ denotes the distance between two neighboring droplets.

A comparison between Eq. (4.20) and the mean distance $d_{n n}$ in Eq. (4.17) yields

$$
\begin{gathered}
\text { experimental: } d_{\mathrm{nn}}=0.51 \mathrm{~mm} \text {, } \\
\text { theoretical: } \lambda_{\mathrm{c}}=0.52 \mathrm{~mm}
\end{gathered}
$$

Eqs. 4.21a and 4.21b show that the theoretical value for $\lambda_{\mathrm{c}}$ agrees within $2 \%$ with the experimental value for $d_{n n}$ which is obtained from the one-dimensional azimuthally averaged LPSD. As mentioned in [8], for the critical thickness $d_{\mathrm{c}}$ of the liquid film on the underside of the top plate it follows

$$
d_{\mathrm{c}}=8.5 \times 10^{-5}\left[\alpha\left(T_{\mathrm{i}}-T_{\mathrm{t}}\right)\right]^{1 / 4} \mathrm{~m} \mathrm{~K}^{-1 / 4} .
$$

Here $\alpha<1$ is a dimensionless accommodation coefficient and $T_{\mathrm{i}}$ denotes the temperature between the surface of the liquid film and the subjacent gaseous phase. With an assumed value of $\alpha=0.1$ and an estimated temperature difference of $T_{\mathrm{i}}-T_{\mathrm{t}}=5 \mathrm{mK}$, the critical thickness of the liquid film is estimated to

$$
d_{\mathrm{c}}=12.7 \times 10^{-6} \mathrm{~m} \text {. }
$$

In [8] the RTI develops with the critical wavelength $\lambda_{c}$ as a lower bound. The liquid film becomes unstable for wavelengths larger than $\lambda_{\mathrm{c}}$. Although the result in Eq. (4.21a) is in good agreement with the theoretical prediction of $\lambda_{c}$, it must be 
Thermophysical properties

Value

$\left(T_{1}=317.650 \mathrm{~K}, T_{2}=317.763 \mathrm{~K}, p_{1}=53.974\right.$ bar,

$p_{2}=36.672$ bar, $y_{2}=14.5 \%$ )

${ }^{\text {a Density }} \rho_{\mathrm{L}}\left(T_{1}, p_{1}\right)$ of liquid $\mathrm{SF}_{6}$

$1.2341 \times 10^{3} \mathrm{~kg} \mathrm{~m}^{-3}$

${ }^{\mathrm{b}}$ Density $\rho_{\mathrm{G}}\left(T_{2}, p_{1}, y_{2}\right)$ of the $\mathrm{SF}_{6}$ and He mixture 455.4

$\mathrm{kg} \mathrm{m}^{-3}$

a Surface tension $\sigma\left(T_{1}, p_{2}\right)$ of liquid $\mathrm{SF}_{6}$

$2.6538 \times 10^{-5} \mathrm{~N} \mathrm{~m}^{-1}$

a Data taken from [52].

b Data taken from [1].

Table 4.6: Thermophysical properties of liquid $\mathrm{SF}_{6}$ and of the gaseous phase of $\mathrm{SF}_{6}$ and He.

considered that $\lambda_{\mathrm{c}}$ is the wavelength at which the liquid film becomes RayleighTaylor unstable while $d_{n n}$ is determined for the case when the instability is already fully developed. In numerical simulations it was shown that regular hexagonal structures were formed by applying the evaporation and the condensation model [8]. The length scale of the hexagonal structures had the critical wavelength $\lambda_{c}$ as a lower bound. The results of the simulations in [8] correspond to the hexagonal droplet pattern which is observed in the $\mathrm{SF}_{6}$ and He mixture.

In contrast to [8], in [10] a temperature dependent surface tension is considered as a stabilizing mechanism for the liquid film. In [10] the critical temperature difference $\Delta T_{\text {lf }}$ across the liquid film on the underside of the top plate is determined to

$$
\Delta T_{\mathrm{lf}}=1.35 \times 10^{8} \mathrm{~d}^{2} \mathrm{Km}^{-2},
$$

where $d$ is the thickness of the liquid film. If a thickness $d=5 \times 10^{-6} \mathrm{~m}$ is assumed similar to the one in Eq. (4.23), then $\Delta T_{\text {lf }}$ is determined to

$$
\Delta T_{1 \mathrm{f}}=4 \mathrm{mK},
$$


which corresponds to the assumed temperature difference in Eq. (4.23). For temperature differences $\Delta T>\Delta T_{\mathrm{lf}}$, the surface of the liquid film remains stable. In [10] the critical thickness of the liquid film is derived in the form

$$
d_{\mathrm{c}}=8.6 \times 10^{-5}\left(T_{\mathrm{i}}-T_{\mathrm{t}}\right)^{1 / 2} \mathrm{~m} \mathrm{~K}^{-1 / 2} .
$$

If the same temperature difference $T_{\mathrm{i}}-T_{\mathrm{t}}=5 \mathrm{mK}$ as for the model in [8] is assumed, then the critical thickness of the liquid film is determined to

$$
d_{\mathrm{c}}=6.1 \times 10^{-6} \mathrm{~m} \text {, }
$$

which is of the same order of magnitude as the thickness in Eq. (4.23). The distinct feature between the model in [8] and in [10] is, that in [10] the liquid film becomes unstable at wavelengths $\lambda>\lambda_{\mathrm{c}} / \sqrt{\epsilon}$, where $\epsilon=\Delta T_{\mathrm{c}}-\Delta T / \Delta T_{\mathrm{c}}$ is the reduced control parameter. That means in [10] the instability occurs at long wavelengths if $\epsilon \rightarrow 0$ while in [8] the instability occurs at a finite wavelength $\lambda_{\mathrm{c}}$. The model in [8] seems to be more applicable to the observed droplet pattern since for the assumption of a constant surface tension under the influence of gravity, hexagonal structures were observed in numerical simulations [8] as well as in experiments [23].

\subsection{Cloud Patterns}

This section describes the cloud-like patterns that were observed in the $\mathrm{SF}_{6}$ and $\mathrm{He}$ mixture. In the following the cloud-like patterns are simply referred to as "cloud patterns". The experimental parameters for the observation of the cloud patterns are given in Table 4.1. During the experiments a liquid phase of $\mathrm{SF}_{6}$ and a gaseous phase of $\mathrm{SF}_{6}$ and He coexisted in the convection cell. Immediately after the bottom 
plate heater is switched on, the droplet formation sets in at the cold top plate. The cloud patterns occur $t \approx 30 \mathrm{~min}$ after the bottom plate heater is switched on. From the observations it is conjectured that the cloud patterns occur in a thin layer above the liquid-vapor interface.

This section is structured as follows. First a qualitative description of the observed cloud patterns is given in Section 4.3.1. It is conjectured that the cloud patterns in the $\mathrm{SF}_{6}$ and $\mathrm{He}$ mixture are formed by nucleated droplets. If this turns out to be true, then the droplets must be formed by the mechanism of homogeneous nucleation since no nucleation sites are contained in the $\mathrm{SF}_{6}$ and He mixture. In the binary gas mixture, $\mathrm{SF}_{6}$ and $\mathrm{He}$ are the condensable and the noncondensable component while in the atmosphere the water vapor and the dry air represent the condensable and the noncondensable component respectively. Since the system has the aforementioned analogies with the earth's atmosphere, the attempt is made to verify if the cloud patterns in the $\mathrm{SF}_{6}$ and $\mathrm{He}$ mixture exhibit characteristic features which are typical for atmospheric clouds. In the literature it is reported that atmospheric clouds show a fractal behavior that is scale invariant and that is observed to occur over a large range of cloud diameters. Therefore a quantitative analysis of the cloud patterns is performed by determining the fractal scaling exponent $D_{\mathrm{p}}$. The result is then compared to the fractal dimensions that are reported in the literature and that were obtained from the fractal analysis of atmospheric cloud structures. For the quantitative analysis of the cloud patterns an image sequence of $n=5000$ images with a frame rate of $5 \mathrm{fps}$ was recorded. At first the cloud regions must be identified in the images. Section 4.3 .2 describes the image processing techniques that were applied in order to perform a segmentation of the cloud patterns and determine the area and the perimeter of the identified cloud regions. An histogram analysis of the identified cloud regions is presented in Section 4.3.2. The result of the area-perimeter 
analysis is presented in Section 4.3.3. A possible explanation for the appearance of the cloud patterns is given. References from the literature are presented that support the hypothesis that the cloud patterns are formed by a homogeneous nucleation of droplets. In order to distinguish the droplets that form the cloud patterns from the droplets that occur on the underside of the top plate, in the following the droplets of the cloud patterns are referred to as "nanodroplets".

\subsubsection{Cloud dynamics}

This section presents a selection of images with different types of cloud patterns that were observed in the $\mathrm{SF}_{6}$ and He mixture. The digital camera focusses slightly above the liquid-vapor interface as shown in Fig. 3.3. The cloud patterns appear dark in the images since the light which is scattered by the cloud patterns is not captured by the objective of the digital camera. The background of the images appears lighter since the incoming light is reflected by the polished bottom plate. As shown in Section A.3. the flow inside the gaseous phase of $\mathrm{SF}_{6}$ and $\mathrm{He}$ is considered to be highly turbulent. It is conjectured that the dynamics of the cloud patterns reveal the turbulent flow inside the gaseous phase.

\section{Cellular patterns}

In Fig. 4.16a and in Fig. 4.16b two images of the cell-like cloud patterns are shown. The cloud cells are bounded by thin dark lines. The number of edges and the size of a cell are always different. Sometimes hexagonal-shaped cells are visible. The cell-like patterns are the most frequent patterns and occur in nearly all images of the image sequence. The diameter of the cells in Fig. 4.16 is estimated to be $2 \mathrm{~mm}$ to $4 \mathrm{~mm}$. 


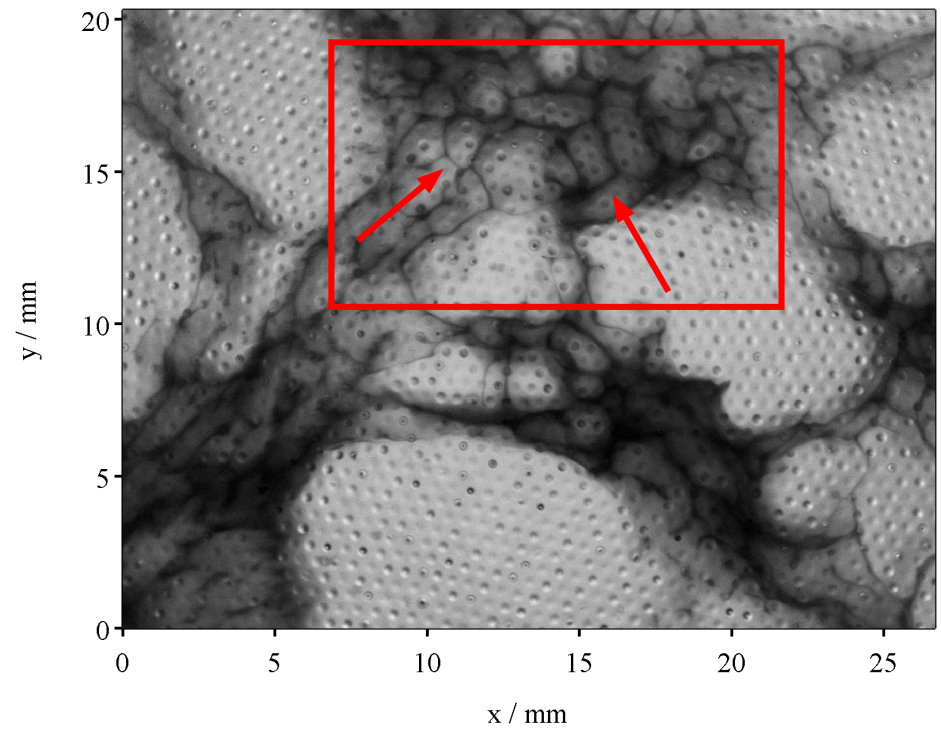

(a)

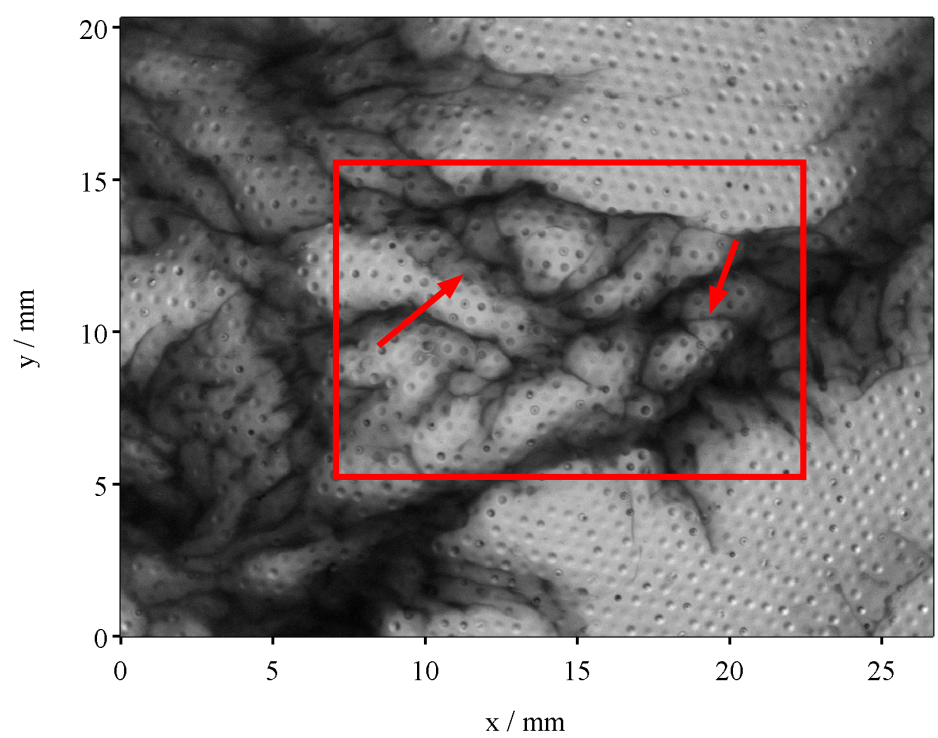

(b)

Figure 4.16: (a) and (b) show cell-like cloud patterns. The cell-like cloud patterns are marked by a red rectangle and are pointed out by red arrows. 


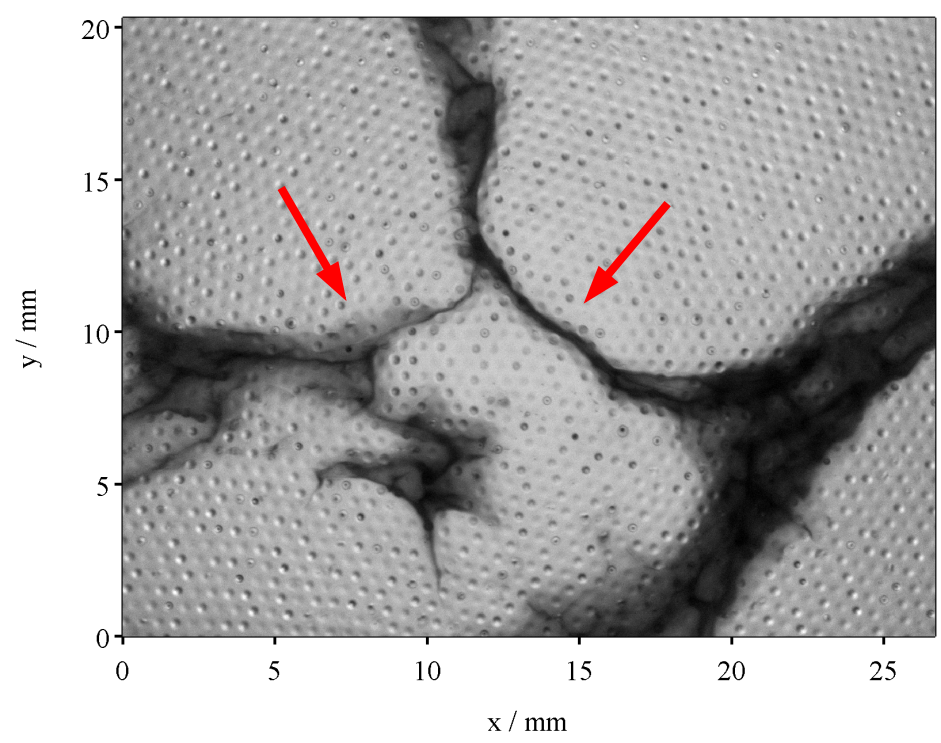

(a)

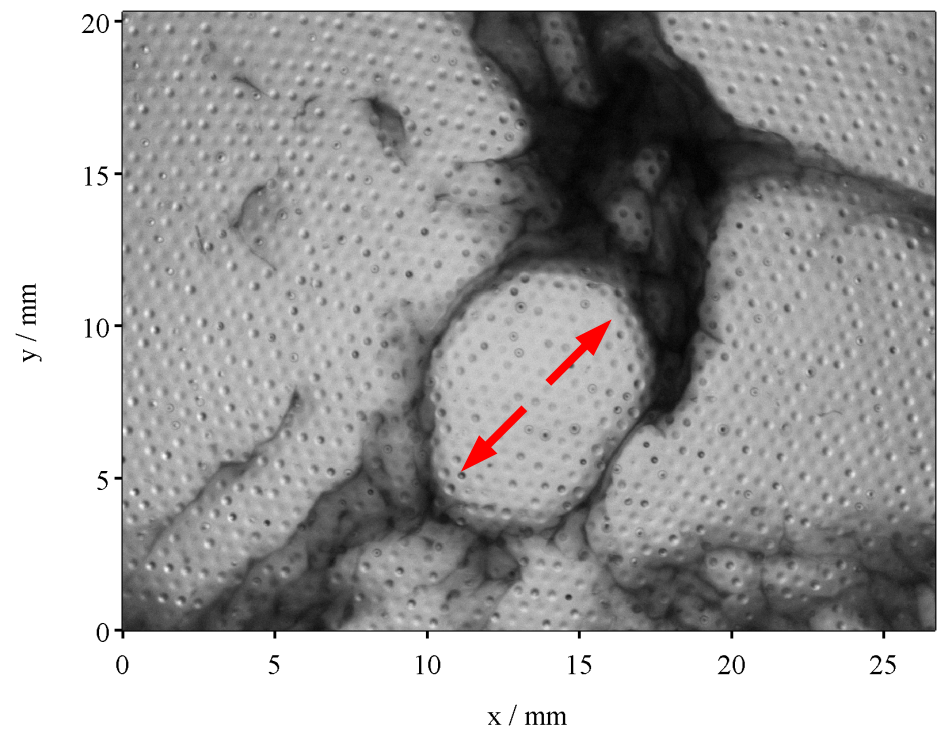

(b)

Figure 4.17: (a) and (b) show two different plume patterns. The plumes move in the direction pointed out by the red arrows.

\section{Plume patterns}

Besides the cellular patterns also plume patterns can be observed as shown in Fig. $4.17 \mathrm{a}$ and in Fig. 4.17b . The plumes are bounded by thick dark lines. Within the plumes no cloud patterns are visible. Two types of plumes are distinguished. The 
bigger plumes like the one in Fig. 4.17a have a diameter larger than $15 \mathrm{~mm}$ and occur every $100 \mathrm{~s}$ to $160 \mathrm{~s}$ in the image area which is captured by the camera. The overall size cannot be determined since the plumes extend beyond the boundaries of the image. The smaller plumes have a diameter of about $10 \mathrm{~mm}$. In Fig. $4.17 \mathrm{~b}$ the plume emerges within a region of existing cloud patterns and then grows in size. If a plume emerges, it spreads and pushes the existing cloud patterns aside. It is assumed that the plumes are caused by the cold downward flowing jets as described in Section 2.5.1. In some images where the plume boundaries are clearly visible, the horizontal plume velocity $U_{\mathrm{p}}$ is estimated by tracking some points on the plume boundaries over time. It is found that the boundaries of the plumes spread with a velocity of $U_{\mathrm{p}} \approx 4 \mathrm{~mm} \mathrm{~s}^{-1}$.

\section{Vortex patterns}

Another type of pattern that is also observed are vortex patterns which are shown in Fig. $4.18 \mathrm{a}$ and in Fig. $4.18 \mathrm{~b}$. This type of pattern occurs roughly every $8 \mathrm{~min}$ in the image region which is captured by the camera. These patterns monitor the complex and turbulent flow inside the gaseous phase. The swirling effect might be caused by warm fluid rising upwards in the center of the vortex while cold fluid is flowing downwards at the outside.

\section{Cloud patterns induced by droplets}

Finally it is observed that the downward falling droplets from the top plate can induce the formation of a new droplet cloud as pointed out by the red arrows in Fig. $4.19 \mathrm{a}$ and in Fig. $4.19 \mathrm{~b}$. The red arrows mark the region where the droplet cloud is formed. The induced droplet cloud is either sucked up by a bigger cloud or dissociates again. From the observations it seems as if the droplet clouds are induced 


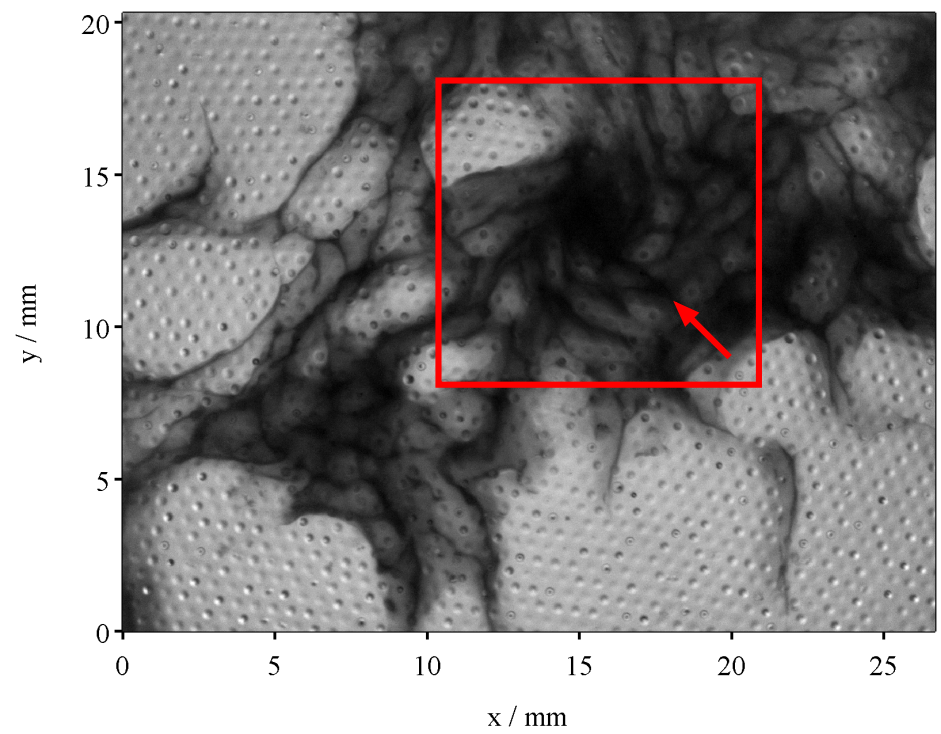

(a)

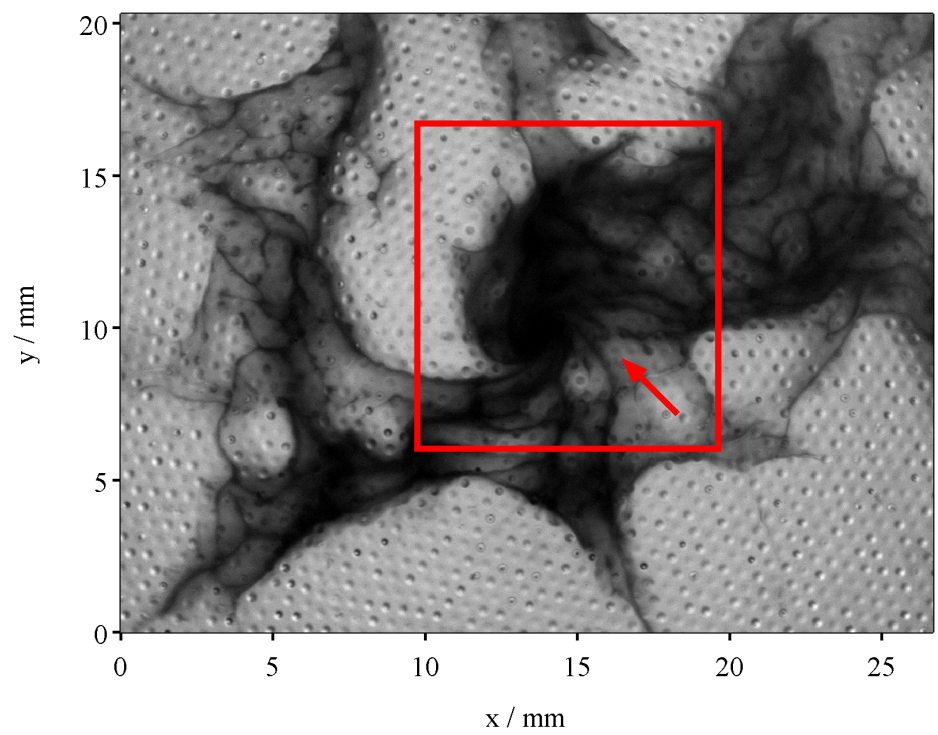

(b)

Figure 4.18: (a) and (b) show two different vortex patterns. The vortex patterns are pointed out by red arrows. 


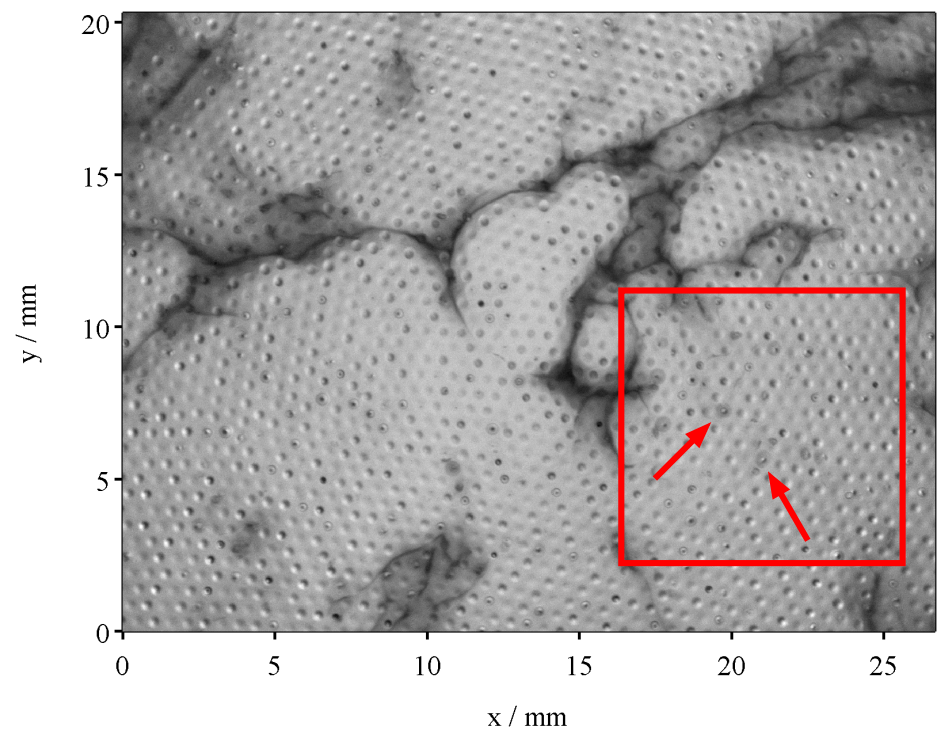

(a)

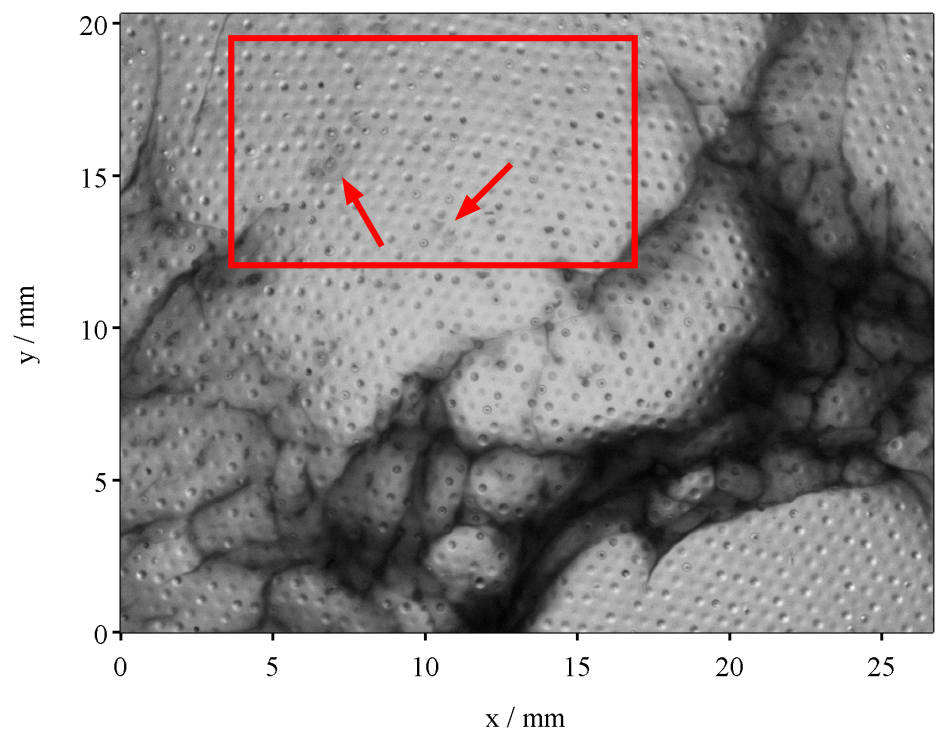

(b)

Figure 4.19: (a) and (b) show small droplet clouds that are induced by the downward falling droplets. The induced droplet clouds are pointed out by red arrows. 
above the liquid-vapor interface where the already existing cloud patterns are located. However, it should be pointed out that not every downward falling droplet induces a new droplet cloud. One method in order to verify that the downward falling droplets can induce the condensation of a small droplet cloud is, to illuminate the convection cell with a laser beam. The condensing droplets which are induced by the downward falling droplets scatter the light of the laser beam and thus become visible to the eye.

\subsubsection{Cloud pattern analysis}

\section{Image analysis}

In order to determine the area and the perimeter of the cloud patterns, the cloud regions in the images must be separated. Therefore an active contour tracking algorithm is employed that can identify the contours of the cloud patterns. Since the active contour tracking algorithm would also detect the contours of the droplet pattern, the effect of the droplet pattern must be reduced at first. For this the images are transformed into the frequency domain by applying the DFT and filtered with an ideal band pass filter. In the end the filtered images still contain the structures of the cloud patterns but the artifacts of the droplets are reduced to a great extent. The filtered images are transformed back into the spatial domain by applying the inverse discrete Fourier transform (IDFT)

$$
f(x, y)=\frac{1}{N M} \sum_{k_{\mathrm{x}}=0}^{N-1} \sum_{k_{\mathrm{y}}=0}^{M-1} F\left(k_{\mathrm{x}}, k_{\mathrm{y}}\right) e^{i 2 \pi\left(\frac{k_{\mathrm{x}} x}{N}+\frac{k_{\mathrm{y}} y}{M}\right)}
$$

as defined in [4, 26].

A two-dimensional active contour tracking algorithm based on the Chan-Vese model [14] is applied to the filtered images in order to identify the contours of the cloud 
regions. In the Chan-Vese model the filtered image $u_{0}(x, y)$ is defined as $u_{0}: \Omega \rightarrow \mathbb{R}$, where $\Omega$ is a bounded open subset of $\mathbb{R}$ with $\partial \Omega$ as its boundary. $C$ is an evolving curve and considered as the boundary $C=\partial \omega$ of an open subset $\omega \subset \Omega$. The region inside and outside of $(C)$ is denoted with $\omega$ and with $\Omega \backslash \bar{\omega}$ respectively. The idea of the Chan-Vese model is to minimize the energy functional

$$
\begin{aligned}
F\left(c_{1}, c_{2}, C\right)= & \mu \text { Length }(C)+v \operatorname{Area}(\omega) \\
& +\lambda_{1} \int_{\omega}\left|u_{0}(x, y)-c_{1}\right|^{2} \mathrm{~d} x \mathrm{~d} y+\lambda_{2} \int_{\Omega \backslash \bar{\omega}}\left|u_{0}(x, y)-c_{2}\right|^{2} \mathrm{~d} x \mathrm{~d} y,
\end{aligned}
$$

which is a modified version of the Mumford-Shah functional for segmentation [50]. Here $\lambda_{1}, \lambda_{2}>0$ and $\mu, v \geq 0$ are fixed parameters. $\lambda_{1}$ and $\lambda_{2}$ weigh the region inside and outside of the contour, $\mu$ and $v$ weigh the length and the area of the contour and $c_{1}$ and $c_{2}$ are the averages of $u_{0}$ inside and outside of $(C)$ respectively. A level set method based formulation of the active contour model is used to solve the minimal partition problem in Eq. (4.29). In the level set method [55] the contour $C \subset \Omega$ is replaced by the zero level set of a Lipschitz function $\phi: \Omega \rightarrow \mathbb{R}$ such that

$$
\left\{\begin{array}{l}
C=\partial \omega=\{(x, y) \in \Omega: \phi(x, y)=0\}, \\
\text { inside }(C)=\omega=\{(x, y) \in \Omega: \phi(x, y)>0\}, \\
\text { outside }(C)=\Omega \backslash \bar{\omega}=\{(x, y) \in \Omega: \phi(x, y)<0\} .
\end{array}\right.
$$

In [14] it is shown that after replacing $C$ with the level set function $\phi$, the variables $c_{1}$ and $c_{2}$ in Eq. (4.29) are given by

$$
\left\{\begin{array}{l}
c_{1}(\phi)=\text { average }\left(u_{0}\right) \text { in }\{\phi \geq 0\}, \\
c_{2}(\phi)=\text { average }\left(u_{0}\right) \text { in }\{\phi<0\} .
\end{array}\right.
$$




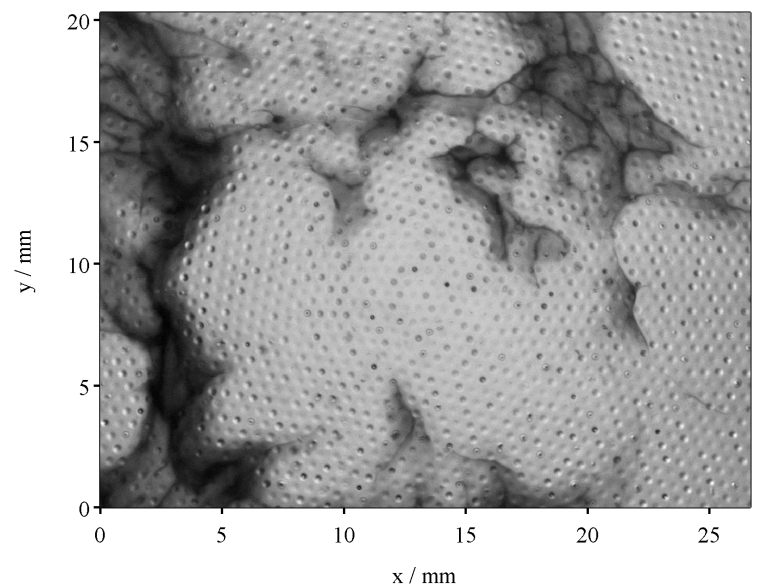

(a1)

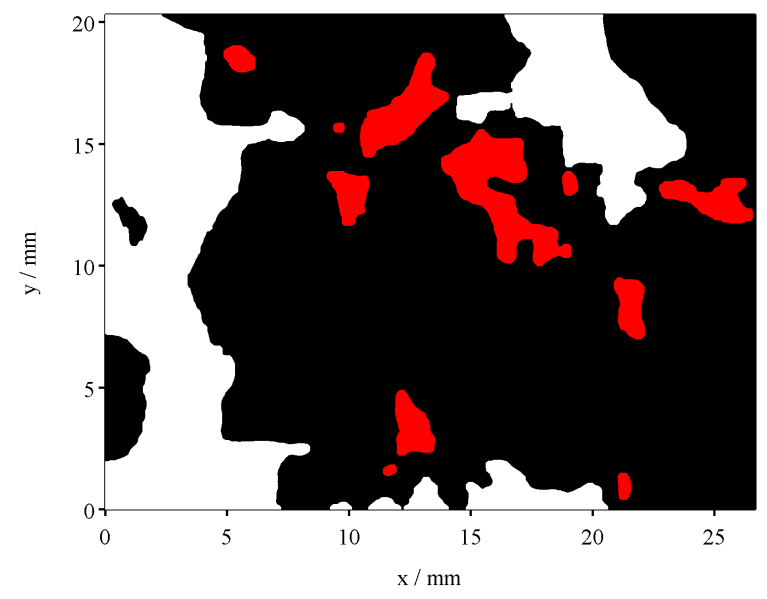

(a2)

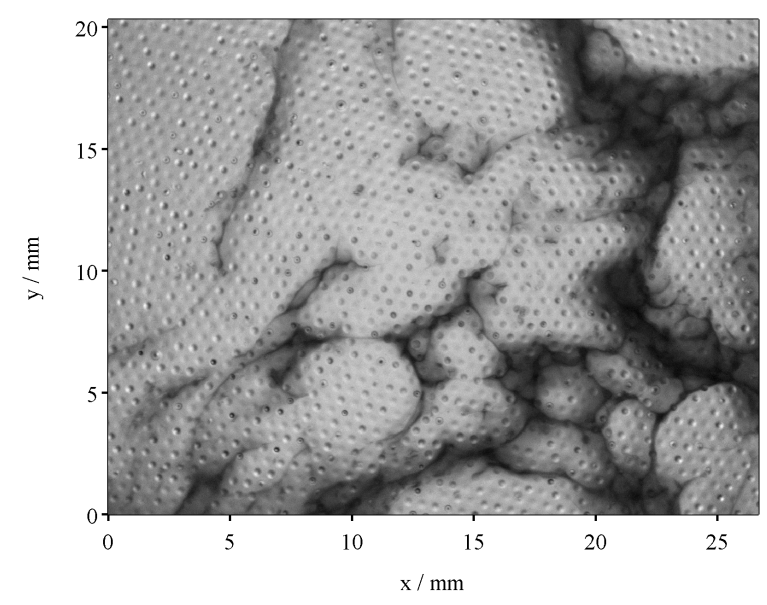

(b1)

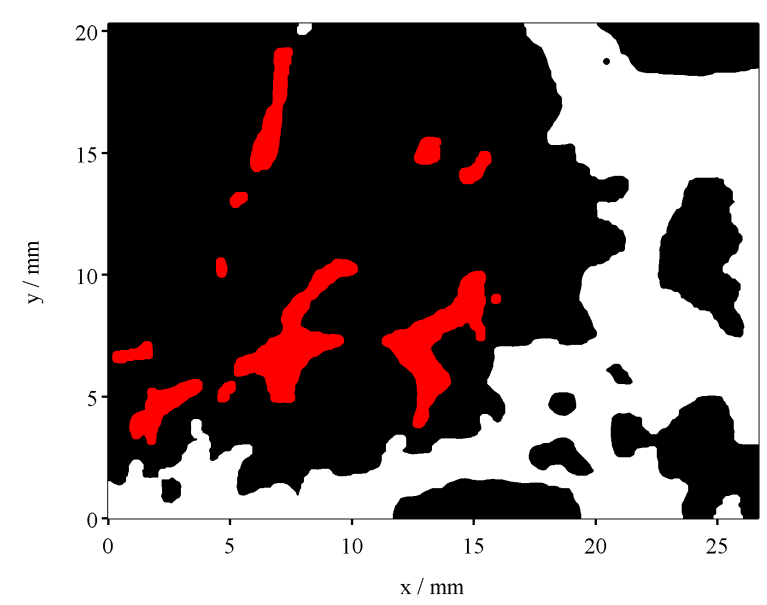

(b2)

Figure 4.20: Output of the two-dimensional active contour tracking algorithm based on the Chan-Vese model. (a1) and (b1) show two background corrected images with the cloud patterns. (a2) and (b2) show the output of the Chan-Vese model. The white areas mark the cloud regions. The inner cloud regions that do not extend beyond the boundaries of the image are marked in red. (a3) and (b3) show an overlay of the original image with the identified cloud regions in (a2) and (b2). The results are obtained with the following parameters for the Chan-Vese model: $\lambda_{1}, \lambda_{2}=1$, $\Delta \mathrm{t}=0.1, \mathrm{~h}=1, \mathrm{n}=10, \mu=0.001$ and $v=0$. Here $\Delta \mathrm{t}$ is the time step, $\mathrm{h}$ the space step and $\mathrm{n}$ defines the number of iterations. 


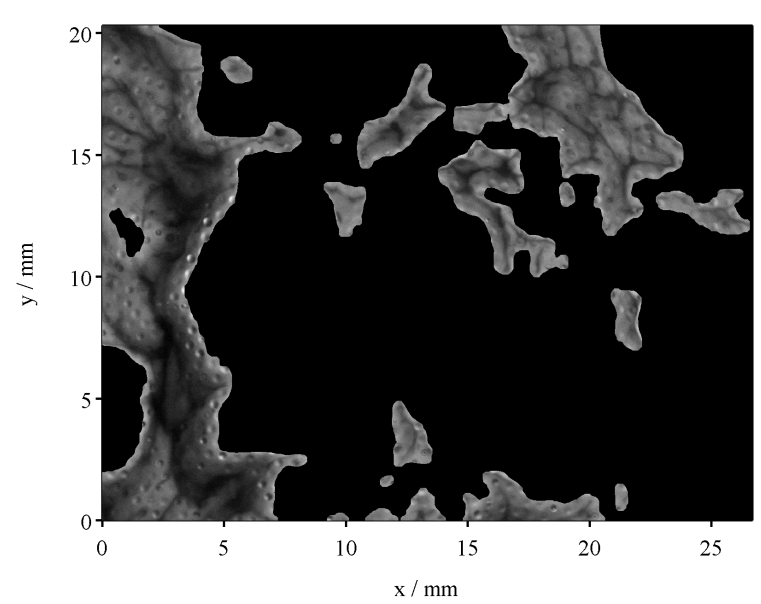

(a3)

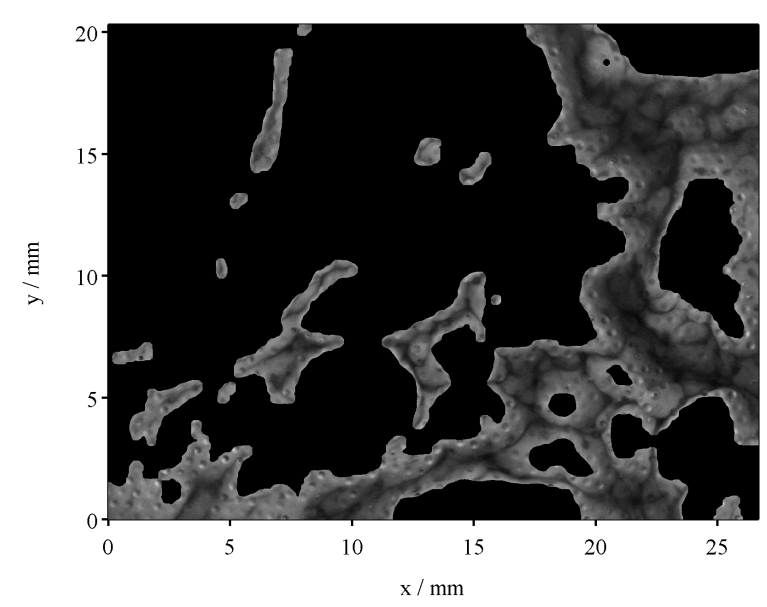

(b3)

Figure 4.20: Output of the two-dimensional active contour tracking algorithm based on the Chan-Vese model. (a1) and (b1) show two background corrected images with the cloud patterns. (a2) and (b2) show the output of the Chan-Vese model. The white areas mark the cloud regions. The inner cloud regions that do not extend beyond the boundaries of the image are marked in red. (a3) and (b3) show an overlay of the original image with the identified cloud regions in (a2) and (b2). The results are obtained with the following parameters for the Chan-Vese model: $\lambda_{1}, \lambda_{2}=1$, $\Delta \mathrm{t}=0.1, \mathrm{~h}=1, \mathrm{n}=10, \mu=0.001$ and $\nu=0$. Here $\Delta \mathrm{t}$ is the time step, $\mathrm{h}$ the space step and $\mathrm{n}$ defines the number of iterations.

A toolbox [27] that contains a numerical implementation of the two-dimensional Chan-Vese model is used to perform the segmentation of the images. The toolbox provides a function to create an initial starting contour $\phi_{0}$ as the level set function $\phi$. The initial curve $\phi_{0}$ and the filtered image $u_{0}$ are then passed to the Chan-Vese algorithm. After a number of iterations, the algorithm outputs the contours of the objects found in the image. According to

$$
\left\{\begin{array}{l}
C C:=\{C \mid(C \in \Omega) \wedge(C \notin \partial \Omega)\} \\
O C:=\{C \mid(C \in \Omega) \wedge(C \in \partial \Omega)\}
\end{array}\right.
$$

the cloud patterns are divided up into closed cloud contours $(C C)$ which do not extend beyond the boundaries $\partial \Omega$ of the image $u_{0}$ and open cloud contours (OC) 
which extend beyond the boundaries of the image and thus cannot be analyzed. The identified cloud patterns are further processed in order to determine the area and the perimeter of the inner cloud regions. As an example two images are shown in Fig. 4.20 which are processed with the Chan-Vese model. Fig. 4.20a1 and Fig. 4.20b1 show the original background corrected images. The output of the Chan-Vese model is shown in Fig. 4.20a2 and in Fig. 4.20b2, The cloud regions are marked in white and the inner cloud regions that do not extend beyond the boundaries of the image are marked in red. Finally in Fig. $4.20 \mathrm{a} 3$ and in Fig. $4.20 \mathrm{b3}$ the identified cloud regions are extracted from the original image in Fig. 4.20a1 and in Fig. 4.20b1. The identified cloud regions contain most of the cloud patterns and it seems as if the Chan-Vese model detects most of the cloud patterns as objects in the images.

\section{Cloud and droplet histograms}

In order to verify that the cloud regions are separated from the droplet regions in the images, a histogram analysis of the intensities of the segmented cloud and the segmented droplet regions is performed. In the following the term cloud images is used for the images that show the cloud patterns and simultaneously the droplet

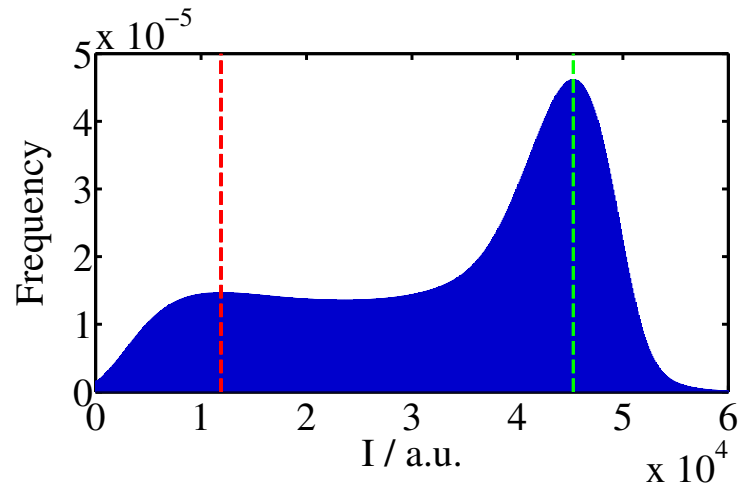

(a)

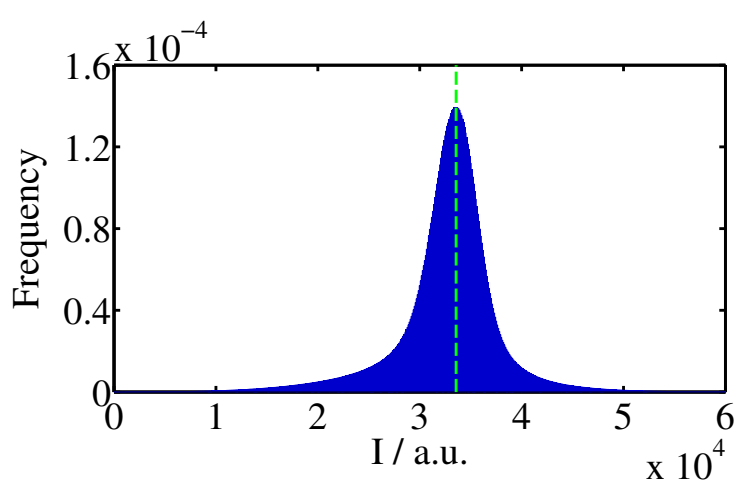

(b)

Figure 4.21: (a) shows the normalized histogram of the cloud images. (b) shows the normalized histogram of the droplet images. 


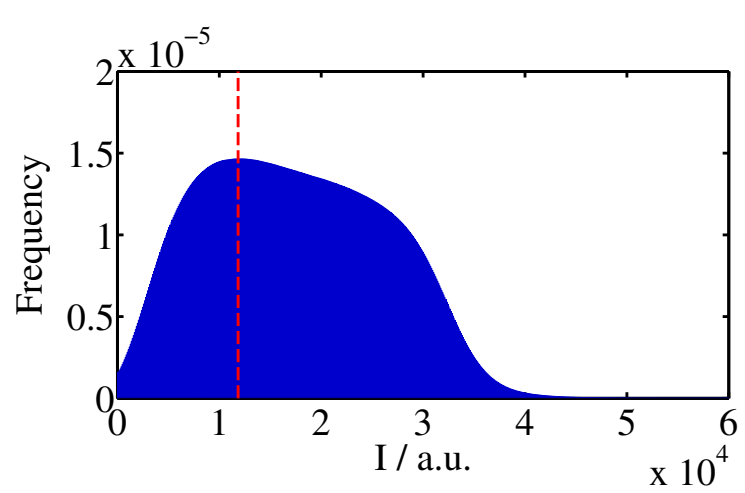

(a)

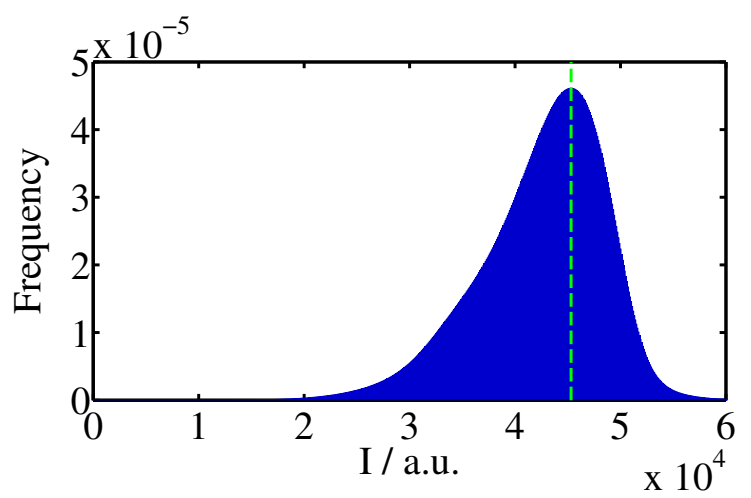

(b)

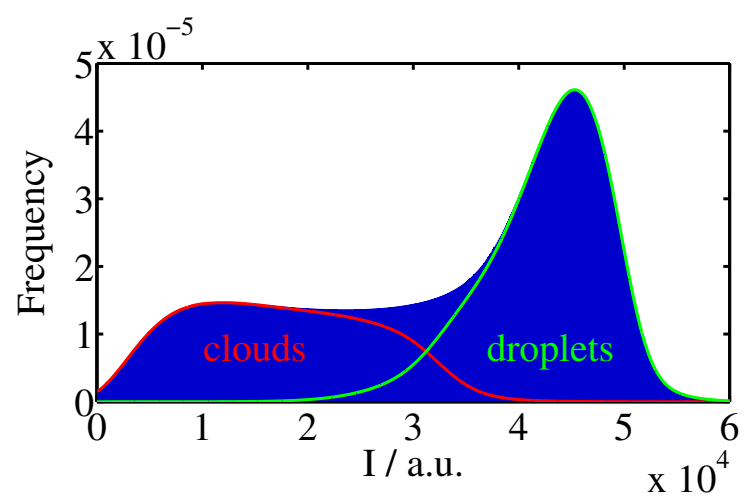

(c)

Figure 4.22: (a) and (b) show the histogram of the separated cloud and the separated droplet regions respectively. In (c) the histograms of (a) and of (b) are added together. The red and the green solid line mark the cloud and the droplet histogram in (a) and in (b) respectively. The histogram in (c) corresponds to the histogram of the cloud images in Fig. 4.21a.

pattern like the ones in Fig. 4.16. For the images like the one in Fig. 4.5 where only the droplet pattern is visible, the term droplet images is used. First the histogram of the cloud and the droplet images is calculated. In the next step the histogram of the separated cloud and the separated droplet regions is determined for comparison. Fig. $4.21 \mathrm{a}$ and Fig. $4.21 \mathrm{~b}$ show the histogram of the cloud and the droplet images respectively. Two peaks can be identified in Fig. $4.21 \mathrm{a}$, a smaller one at $I=1.2 \times 10^{4}$ and a larger one at $I=4.5 \times 10^{4}$ which are marked by a red and a green dashed line 
respectively. The smaller peak is caused by the intensities of the cloud patterns while the larger peak results from the intensities of the droplet pattern. In Fig. $4.21 \mathrm{~b}$ only one peak exists at $I=3.4 \times 10^{4}$ which is marked by a green dashed line. The peak is caused by the droplet pattern since no cloud patterns appear in the droplet images. The histogram of the separated cloud and the separated droplet regions is shown in Fig. $4.22 \mathrm{a}$ and in Fig. $4.22 \mathrm{~b}$ respectively. In Fig. 4.22a a peak can be identified at $I=1.2 \times 10^{4}$ which is marked by a red dashed line. The peak in Fig. $4.22 \mathrm{~b}$ is located at $I=4.5 \times 10^{4}$ and is marked by a green dashed line. The position of the peak in the segmented cloud and the segmented droplet histogram corresponds to the position of the peaks in Fig. 4.21a. Fig. 4.22 shows the histogram when both histograms in Fig. $4.22 \mathrm{a}$ and in Fig. $4.22 \mathrm{~b}$ are added together. The individual histograms of the separated cloud and the separated droplet regions are illustrated by a red and a green solid line respectively. The result in Fig. 4.22c shows that the Chan-Vese model is suitable in order to identify the cloud patterns in the images.

\subsubsection{Discussion}

\section{Fractal scaling exponent}

After the images are segmented into the cloud and the droplet regions, the area $A$ and the perimeter $c$ of the inner cloud regions in Fig. 4.20a2 and in Fig. $4.20 \mathrm{~b} 2$ are determined by counting the number of pixels of the area and the contours of the inner cloud regions respectively. A detailed description of the method can be found in [24]. A total number of $n_{C C}=32973$ inner cloud regions were identified in the image sequence. If the area and the perimeter are known, then the area-perimeter relation

$$
c \sim \sqrt{A}^{D_{\mathrm{p}}}
$$




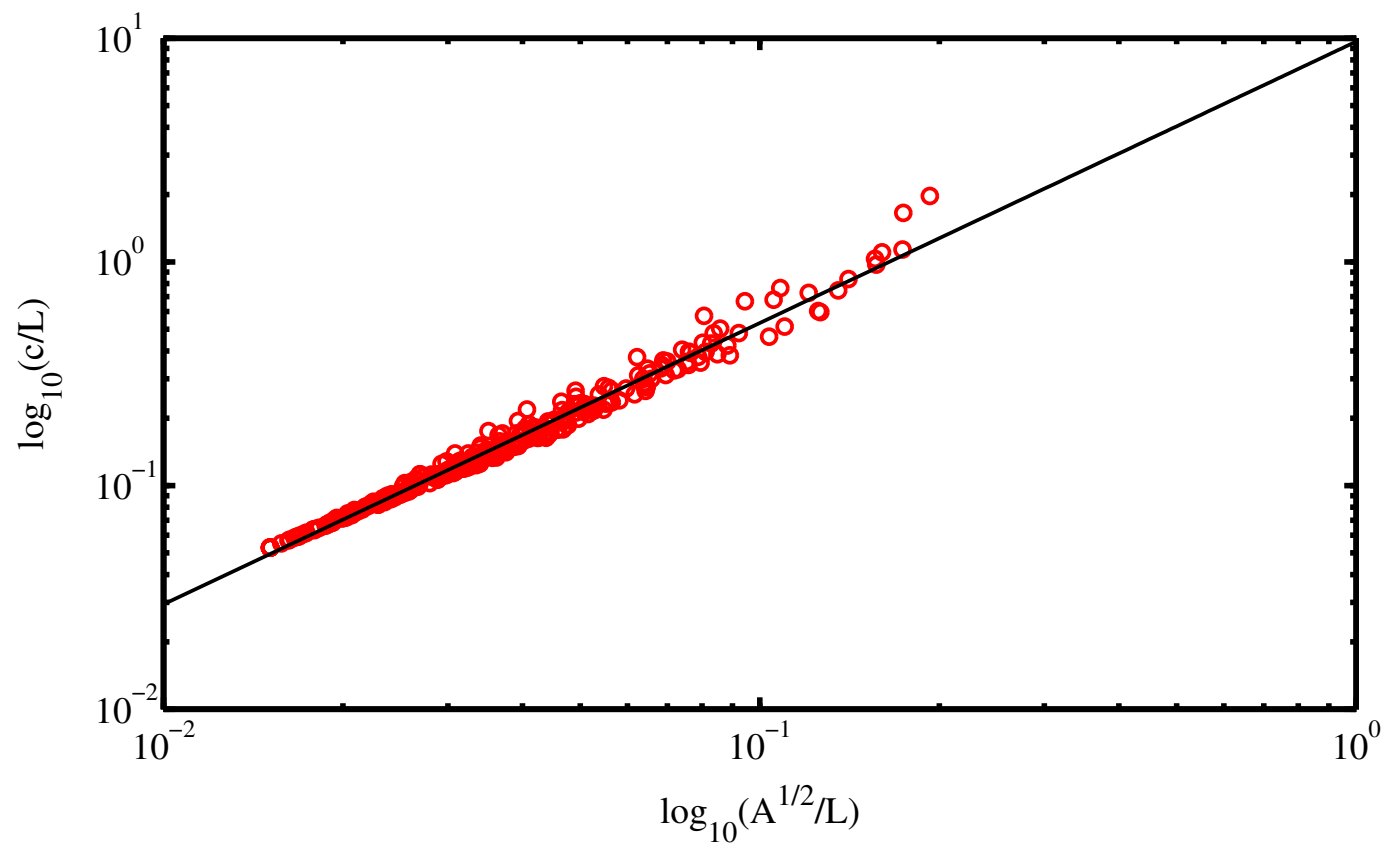

Figure 4.23: Log-log plot of the perimeter $c$ versus the area $A$ of the inner cloud regions.

is used to determine the fractal scaling exponent $D_{\mathrm{p}}$ as described in [73]. The fractal scaling exponent which is also known as the fractal dimensionality describes the selfsimilarity of the system. Fig. 4.23 shows the $\log -\log$ plot of the perimeter $c$ versus the area $A$ of the inner cloud regions. The open red circles mark the area-perimeter ratio of the identified inner cloud regions and the solid black line represents the fit with a power law by applying Eq. (4.33). The fractal scaling exponent $D_{\mathrm{p}}$ is found to be

$$
D_{\mathrm{p}}=1.26
$$

Since the width of the image area is restricted, this prevents the analysis of bigger cloud regions. Therefore the scaling range over which the cloud patterns were analyzed, comprises only one order of magnitude. The values for $D_{\mathrm{p}}$ found in the literature differ from the value in Eq. (4.34) and are slightly larger. In [46] the areaperimeter relation was applied to investigate the cloud and the rain areas in satellite 
and radar data. The data were fitted by a power law with $D_{\mathrm{p}}$ as the fractal dimension of the perimeter. In [46] it was supposed that if the cloud areas had a characteristic length scale, then the cloud areas could be considered to consist of large-scale and small-scale structures. The different scales would result in different area-perimeter relations which in turn would result in different values of $D_{\mathrm{p}}$. If this was the case, then this would have allowed to distinguish between processes which occur on large scales and processes which occur on small scales [46]. However, if no characteristic length scale exists, then the perimeter scales with the area as shown in Eq. (4.33). In [46] the analysis of the cloud and the rain areas resulted in a value of $D_{\mathrm{p}} \sim 1.35$ that is larger than the value in Eq. (4.34). The analysis in [46] revealed no scalebreak over a range of nearly 4 orders of magnitude. Since the cloud patterns that are described in this thesis occur on much smaller length scales, this would allow to extend the analysis of the fractal dimension down to scales of the order of $\mathcal{O}\left(10^{-1}\right)$ or $\mathcal{O}\left(10^{-2}\right)$ in order to show that the range of the scale-less behavior may continue down to length scales of the order of $\mathcal{O}(\mathrm{cm})$ or even below. Another fractal analysis of convective storms was performed in [62]. Here a fractal shape was observed for large clouds and an area-perimeter analysis yielded a value of $D_{\mathrm{p}} \sim 1.36$. The analyzed scale ranged from $1 \mathrm{~km}$ to $10^{2} \mathrm{~km}$. A sharp crossover is observed which would indicate the existence of a characteristic scale but is presumably caused by a particle drift. Another study of the area-perimeter relation of stratocumulus and fair weather clouds was conducted in [13]. Here it was found that the probability distribution of cloud areas and cloud perimeters approximately follows a power-law with a different fractal dimension for each cloud type. For the area-perimeter relation of stratocumulus clouds a value of $D_{\mathrm{p}} \sim 1.4$ was found. However, for fair weather clouds two different scalings were found so that the area-perimeter relation could be better approximated by two power-laws with two different scaling exponents. For 
cloud bases less than $0.5 \mathrm{~km}$ in diameter a value of $D_{\mathrm{p}} \sim 1.34$ was found while for larger clouds the value was determined to $D_{\mathrm{p}} \sim 1.55$. However, in [47] the aforementioned change in the fractal dimension was attributed to the effect of data resolution. In [64] the fractal analysis was applied in large-eddy simulations (LES) of geometric properties of cumulus clouds. The three-dimensional LES models confirmed the fractal scaling laws from the area-perimeter analysis of the cloud and the rain areas in satellite and radar data. A short summary of the aforementioned experimental results can be found in [33].

The reason why the value for the fractal dimension in Eq. (4.34) is lower than the values which are reported in the literature, is because $D_{\mathrm{p}}$ depends strongly on the image processing techniques that were applied to identify the inner cloud regions as described in Section 4.3.2. The droplet pattern at the top plate makes it difficult to perform a segmentation of the images without applying an ideal band pass filter. The active contour tracking algorithm detects the cloud regions in the images but it does not reveal the complexity and the raggedness of the cloud contours in detail. The identified contours are smoothed out which results in a smaller value of $D_{\mathrm{p}}$. If the raggedness of the cloud regions can be resolved in more detail, then this would result in a larger perimeter $c$ while the area $A$ of the cloud region remains approximately the same. This would result in a larger value of $D_{\mathrm{p}}$. Therefore it is conjectured that the true fractal dimension $D_{\mathrm{p}}$ of the cloud patterns is larger than the value in Eq. (4.34). As a consequence the image analysis of the cloud patterns must be improved.

If further experiments confirm that the observed cloud patterns are formed by nanodroplets, then this would allow to extend the fractal analysis of two-dimensional cloud structures down to length scales of the order of $\mathcal{O}(\mathrm{cm})$ and below. This might help to answer the question whether the scale-less behavior of the area-perimeter 
relation continues down to distances of the order of $\mathcal{O}(\mathrm{cm})$ as conjectured in [46]. The applicability of a fractal model would then be extended to a larger range of meteorological significant distances from below $1 \mathrm{~cm}$ up to $10^{4} \mathrm{~km}$ [46].

\section{Cloud patterns}

In this section it is discussed why it is likely that the observed cloud patterns are caused by the nucleation of a new phase and not by other effects that might also occur in the fluid mixture like critical opalescence [17, 67] or a change in the refractive index of the fluid mixture that produces caustics [60]. If the cloud patterns occur due to the nucleation of a new phase, then the new phase must be formed by a homogeneous nucleation since no nucleation sites are contained in the $\mathrm{SF}_{6}$ and He mixture which would allow a heterogeneous nucleation in the bulk of the gaseous phase. Furthermore a region of supersaturation must exist in the bulk of the gaseous phase in order that a stable cluster can be formed by a homogeneous nucleation [69, 70, 71]. A few observations have been made that support the assumption that the cloud patterns are formed by nanodroplets that are induced by a homogeneous nucleation. First it is observed that the edges of the patterns in Fig. 4.16 and Fig. 4.17 do not seem to result from a fluctuation in the refractive index as would be the case if critical opalescence is responsible for the appearance of the patterns. Instead the edges of the patterns seem to move with the turbulent flow and the shape of the edges is roughly preserved while the patterns are moving through the image plane. It was found that the moving boundaries of the plumes spread with a velocity of

$U_{\mathrm{p}} \approx 4 \mathrm{~mm} \mathrm{~s}^{-1}$. Second it is observed that the downward falling droplets can induce the condensation of a small droplet cloud as described in Section 4.3.1. In order that a droplet cloud can be formed, a layer of supersaturated $\mathrm{SF}_{6}$ vapor must exist above the liquid phase of $\mathrm{SF}_{6}$. This layer is caused by the mass flux from the surface of 
the liquid phase of $\mathrm{SF}_{6}$ to the top plate. It is further assumed that supersaturated conditions $(S>1)$ in this layer lead to a permanent nucleation of nanodroplets. For normal saturated conditions $(S=1)$ the nanodroplets would appear and disappear but would not form a stable cluster. When a droplet from the top plate falls down, before it impacts into the liquid pool above the bottom plate, it penetrates through the supersaturated $\mathrm{SF}_{6}$ vapor layer. Since the downward falling droplet has a lower surface temperature than the surrounding vapor, the droplet cools the supersaturated $\mathrm{SF}_{6}$ vapor very quickly and induces the condensation of nanodroplets. The nanodroplets rapidly grow to a visible size and can be visualized by a laser beam. However, not every downward falling droplet produces a small droplet cloud. It is conjectured that the downward falling cold plumes wash the already existing cloud patterns away and wipe out the supersaturated $\mathrm{SF}_{6}$ vapor layer. The supersaturated conditions must first be reestablished before a new droplet cloud can be induced by the downward falling droplets. This scenario is schematically illustrated in Fig. 4.24 . The cloud patterns occur above the liquid-vapor interface which is marked by a dashed horizontal black line. The cold downwelling plumes that are marked by the curved dotted blue arrows wash the cloud patterns away as can be seen in Fig. 4.17. The droplet pattern is represented by the corrugated pattern on the underside of the top plate. It is illustrated how the downward falling droplets can induce the condensation of a new droplet cloud as can be seen in Fig. 4.19.

A supersaturation is essential in order that a homogeneous nucleation can occur. The existence of a supersaturated $\mathrm{SF}_{6}$ vapor layer above the liquid-vapor interface would explain why the cloud patterns occur only in a thin layer above the liquid-vapor interface and not in the bulk of the gaseous phase. In the following paragraph an explanation is given why it is likely that a supersaturated $\mathrm{SF}_{6}$ vapor phase forms above the liquid-vapor interface. In Eqs. (A.9) and (A.11) it is shown that the critical tem- 


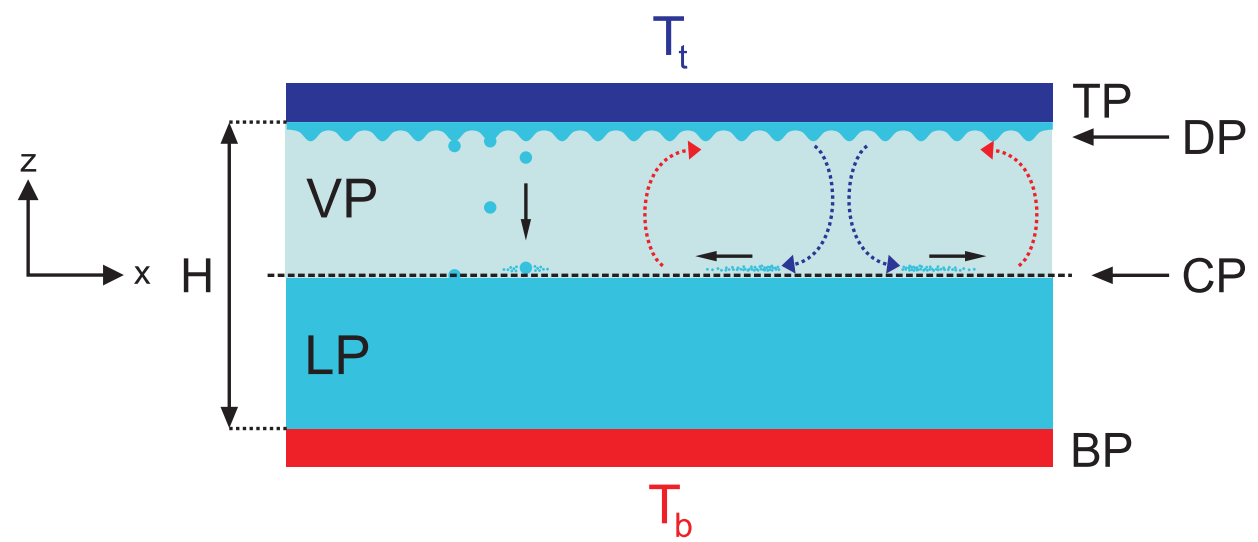

Figure 4.24: Schematic drawing of the droplet pattern and the cloud patterns. BP $=$ bottom plate, $\mathrm{LP}=$ liquid phase, $\mathrm{TP}=$ top plate, $\mathrm{VP}=$ vapor phase, $\mathrm{CP}=$ cloud patterns, $\mathrm{DP}=$ droplet pattern, $T_{\mathrm{b}}=$ bottom plate temperature, $T_{\mathrm{t}}=$ top plate temperature.

perature difference $\Delta T_{\mathrm{c}}$ for the onset of the thermal convection in the liquid phase of $\mathrm{SF}_{6}$ as well as in the gaseous phase of $\mathrm{SF}_{6}$ and $\mathrm{He}$ is of the order of $\mathcal{O}\left(10^{-5} \mathrm{~K}\right)$. This is approximately four orders of magnitude smaller than the applied temperature difference $\Delta T$. Similarly $R a$ for the liquid phase of $\mathrm{SF}_{6}$ as well as for the gaseous phase of $\mathrm{SF}_{6}$ and $\mathrm{He}$ is found to be of the order of $\mathcal{O}\left(10^{7}\right)$ as shown in Eqs. (A.8) and (A.10). Since $R a_{\mathrm{L}} \gg R a_{\mathrm{c}}$ and $R a_{\mathrm{V}} \gg R a_{\mathrm{c}}$, it is conjectured that the convection in the liquid phase of $\mathrm{SF}_{6}$ as well as in the gaseous phase of $\mathrm{SF}_{6}$ and He is highly turbulent. From simulations of turbulent RBC it is known that the temperature drop occurs in the thermal boundary layers and that the mean temperature in the bulk of the fluid remains isothermal [6, 12, 20]. The possible temperature profile $T(z)$ in the convection cell is schematically illustrated in Fig. 4.25 . Here $T_{\mathrm{b}}$ and $T_{\mathrm{t}}$ denote the temperature at $z=0$ and at $z=H$ respectively. The position of the droplet pattern and the cloud patterns are marked by black arrows. If $R a<R a_{\mathrm{c}}$, then heat is transported by thermal diffusion from the bottom to the top plate and the temperature profile in $z$-direction is assumed to be linear as marked by the solid and the dashed blue line. On the other hand if $R a \gg R a_{\mathrm{c}}$, then the convection in the liquid as well 


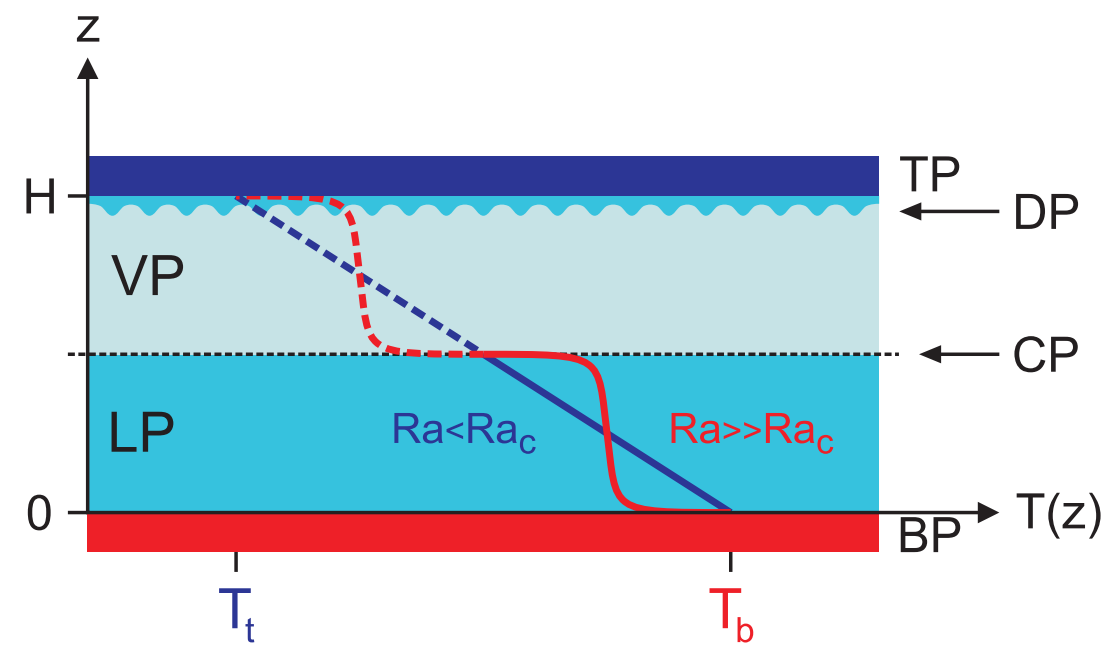

Figure 4.25: Schematic drawing of the temperature profile $T(z)$ in the convection cell. $\mathrm{BP}=$ bottom plate, $\mathrm{LP}=$ liquid phase, $\mathrm{TP}=$ top plate, $\mathrm{VP}=$ vapor phase, $\mathrm{CP}=$ cloud patterns, $\mathrm{DP}=$ droplet pattern, $T_{\mathrm{b}}=$ bottom plate temperature, $T_{\mathrm{t}}=$ top plate temperature.

as in the gaseous phase is highly turbulent and the heat is transported by upwelling and downwelling plumes. The mean temperature in the bulk of the liquid and the gaseous phase remains isothermal and a temperature drop occurs only in the thermal boundary layers as marked by the solid and the dashed red line. It is supposed that the temperature drop in the lower thermal boundary layer of the gaseous phase causes an isobaric cooling of the saturated $\mathrm{SF}_{6}$ vapor phase. From the literature it is known that an isobaric cooling results in a supersaturation [70, 71]. Therefore it is likely that a layer of supersaturated $\mathrm{SF}_{6}$ vapor forms above the liquid-vapor interface. This assumption is supported by the literature. In numerous studies it was shown that a supersaturation can be achieved in vapor-gas and in vapor-liquid systems which results in a homogeneous nucleation of droplets. In many of these studies the nucleation rate was measured in order to determine the critical supersaturation. Most of the systems contained a condensable gas that forms a liquid and a vapor phase and a noncondensable gas that serves as a background or carrier gas. So the physical model systems used in the literature are similar to the one used in this 
thesis. A review of existing measurement methods and experimental techniques in order to investigate the homogeneous nucleation in vapor-gas and in vapor-liquid systems can be found in [3, 30, 38]. In the following paragraph a few references are mentioned where a supersaturation was achieved in a two-phase binary gas mixture that caused a homogeneous nucleation of droplets. In some of these studies the condensation of droplet clouds and also the formation of fog was observed.

In [36, 37] a thermal diffusion cloud chamber (TDCC) was employed in order to measure the critical supersaturation for a homogeneous nucleation for eight different substances with helium and hydrogen as carrier gases. In a TDCC one-dimensional diffusion takes place through a carrier gas from a warm bottom to a cold top plate. In [36] the substances under investigation form a liquid and a vapor phase. If the vapor evaporates from the liquid surface, it diffuses upwards through the carrier gas and condenses at the cold top plate. The degree of supersaturation in the chamber was obtained by dividing the local partial pressure of the vapor by the corresponding equilibrium vapor pressure at the local temperature. The level of supersaturation in the chamber was adjusted by varying the temperature difference between the bottom and the top plate. Since the density in the chamber decreases from the bottom to the top, the density variation prevents a convection and a continuous supersaturation is maintained in the chamber. In the TDCC a layer of supersaturation forms below the top plate and not above the liquid-vapor interface. Other studies on critical supersaturation were performed in [29, 31]. Here a high pressure cloud chamber (HPCC) was used to measure the critical supersaturation for the homogeneous nucleation of four different substances in the presence of helium and hydrogen as carrier gases. It was observed that the nucleation rate depends on the total pressure in the chamber. The critical supersaturation increased when the total pressure in the chamber was increased. This implies that the homogeneous nucleation rate decreases when 
the total pressure in the chamber is increased. Furthermore it was found that for all substances the dependence of the critical supersaturation on the total pressure is dependent on the nature of the carrier gas. A similar experiment was performed in [75]. Here the critical supersaturation was measured for $\mathrm{SF}_{6}$ with helium as a carrier gas. Again it was found that the nucleation rate increases if the total pressure in the chamber is increased. In [21] it is reported that if the experimental conditions are same, then the degree of supersaturation that is required for a homogeneous nucleation in the pure vapor phase of a condensable component is lowered if a carrier gas is present. It is also reported that the carrier gas promotes a nucleation of droplets. Moreover if the carrier gas has good heat conducting properties, then the latent heat that is released by the condensation and the growth of the droplets can be transported away more easily.

In [34] the condensation of steam in the presence of air as a noncondensable gas was investigated. Therefore the flow of an air-steam mixture was directed into a rectangular channel over a condensing plate. In a horizontal position and for very small inclination angles, a condensate film with pendant drops was developing on the underside of the plate. It was observed that small "wakes" of fog formed downstream of the pendant droplets. Similar experiments were conducted in [16]. Here heat transfer measurements for the condensation of steam on the underside of a horizontal surface with air and helium as noncondensable gases are described. A fog or mist formation in the bulk of the experimental apparatus was observed visually. The formation of a fog was also reported in [11]. The experiments were conducted in a cell that contained a liquid and a vapor phase of helium. If the evaporation at the liquid-vapor interface was strong enough, the helium vapor was supercooled and underwent a homogeneous nucleation that resulted in the formation of a dense fog. Model calculations revealed that the strongest supercooled region is expected at the 
liquid-vapor interface where large jumps of the temperature of the gas occur. It was assumed that the large temperature jumps at the liquid-vapor interface caused the supercooled state of the helium gas. Finally in [63] the formation of clouds in a TDCC was studied in numerical simulations. The clouds were formed by the homogeneous condensation of a vapor phase in the presence of a noncondensable gas. In order to study the effect of natural convection on the homogeneous condensation in a TDCC, the Rayleigh number $R a$ was varied from 314 to $10^{4}$ in a system of 1-propanol and helium as a carrier gas. The temperature, supersaturation, mass fraction and nucleation rate profiles were determined numerically. The nucleation plane formed at about $3 / 4$ of the height of the chamber where the critical supersaturation reached its maximum. For the highest Rayleigh number $R a=10^{4}$ only localized zones with a large nucleation rate were observed. The nucleation occurred in these zones due to low temperatures and large supersaturation values.

If the results in the literature are compared to the results obtained for the $\mathrm{SF}_{6}$ and He mixture in this thesis, then it can be concluded that a homogeneous nucleation is likely to happen in the gaseous phase of $\mathrm{SF}_{6}$ and He as reported in [75]. Since no nucleation sites are contained in the $\mathrm{SF}_{6}$ and He mixture, the cloud patterns must be formed by the mechanism of homogeneous nucleation. It is conjectured that a localized region of a supersaturated $\mathrm{SF}_{6}$ vapor phase develops above the liquid-vapor interface which is caused by the temperature drop in the thermal boundary layers. If the degree of supersaturation exceeds a critical value, a homogeneous nucleation is likely to happen which results in the condensation of nanodroplets that accumulate to form the cloud patterns. This assumption is supported by the study in [11] where large temperature jumps at the liquid-vapor interface caused a local supercooling of the helium vapor which caused a homogeneous condensation and the formation of a dense fog. It should be mentioned that the observations made in the $\mathrm{SF}_{6}$ and 
He mixture are so far only qualitative and do not reveal the true nature of the cloud patterns. Further experiments must be carried out in order to clarify if the cloud patterns are formed by nanodroplets which result from the homogeneous nucleation in a supersaturated $\mathrm{SF}_{6}$ vapor phase or if other effects are causing these patterns. Therefore it is suggested to build a new experimental apparatus that allows to investigate the cloud patterns by the method of dynamic light scattering. A suggestion for a potential experimental setup is presented in Section 5.2 . 


\section{Chapter 5}

\section{Conclusion}

\subsection{Summary}

In this thesis turbulent RBC experiments were performed in a two-phase binary gas mixture. A mixture of $\mathrm{SF}_{6}$ and $\mathrm{He}$ was employed as a physical model system. The condensable gas $\mathrm{SF}_{6}$ forms a liquid and a vapor phase while the noncondensable gas He remains in the gaseous phase. When a temperature difference is applied between the bottom and the top plate, two intriguing observations are made.

The first observation is that a liquid film begins to condense on the underside of the top plate what results in the formation of a very regular hexagonal droplet pattern. The temporal evolution of the droplet pattern was quantified by determining the bond orientational order parameter $\phi_{6}$, the average droplet fall-down period $\tau_{\mathrm{fd}}$ and the mean distance $d_{n n}$ between to neighboring droplets. The analysis of $\phi_{6}$ and $\tau_{\mathrm{fd}}$ revealed that a stable mass flux is essential in order to maintain the hexagonal symmetry of the droplet patten. It is conjectured that the droplet pattern at the cold top plate occurs due to a Rayleigh-Taylor instability. Two models are presented that provide an explanation of how a liquid film on the underside of a horizontal plate can be 
stabilized. For the model that considers the evaporation and the condensation as a stabilizing mechanism, numerical simulations showed that regular cell structures in the form of hexagons are formed on a length scale that is similar to the one obtained for the hexagonal droplet pattern in the $\mathrm{SF}_{6}$ and He mixture.

The second observation is that cloud patterns occur in a thin layer above the liquidvapor interface. It is conjectured that a homogeneous nucleation of nanodroplets occurs in a thin layer of a supersaturated $\mathrm{SF}_{6}$ vapor phase which forms above the liquid-vapor interface. The nanodroplets accumulate to form the cloud patterns. Several references are presented that support the assumption that a layer of supersaturation forms above the liquid-vapor interface which is caused by a temperature drop in the thermal boundary layers. A quantitative analysis of the observed cloud patterns is performed by determining the area-perimeter relation which results in a fractal dimension $D_{\mathrm{p}}$ that is somewhat smaller compared to the value that is obtained from the analysis of cloud and rain areas in satellite and radar data. The area-perimeter relation revealed a scale-less behavior of the cloud patterns over one order of magnitude. The deviation of the value of the fractal dimension from the values reported in the literature is attributed to the lack of precision in resolving the raggedness of the cloud patterns.

To conclude, a physical model system is presented in this thesis that can be employed to study the formation and the dynamics of cloud patterns in a turbulent RBC experiment at moderate $R a$ numbers. The physical model system has the following analogies with the earth's atmosphere. The binary gas mixture is composed of $\mathrm{SF}_{6}$ as a condensable and $\mathrm{He}$ as a noncondensable component. In the atmosphere the water vapor represents the condensable and the dry air the noncondensable component. The nanodroplets in the $\mathrm{SF}_{6}$ and He mixture are formed by a homogeneous nucleation since no nucleation sites are contained in the gas mixture. In the atmosphere 
droplets are formed by a heterogeneous nucleation since the dry air contains a lot of nucleation sites. Besides the nucleation mechanism also the $R a$ in the convection cell and in the atmosphere differ by several orders of magnitude. For the convection cell $R a$ is estimated to be of the order of $\mathcal{O}\left(10^{7}\right)$ while in atmospheric convection $R a$

can be as large as $10^{20}$ [22]. Furthermore it must be considered that the processes in the atmosphere are much more complicated compared to the simple physical model system presented in this thesis.

\subsection{Outlook}

Since it is not entirely clarified why the cloud patterns do appear, further experiments must be carried out that could reveal the true nature of these patterns. If it is verified that the cloud patterns are formed by nanodroplets, then the $\mathrm{SF}_{6}$ and $\mathrm{He}$ mixture might be suitable as a model system in order to study the formation and the dynamics of cloud patterns in a turbulent RBC experiment. Therefore it is suggested to build a new experimental apparatus that allows for lateral optical access to the convection cell in order to perform scattering experiments. In Fig. 5.1 a simple schematic drawing for a new experimental setup is presented. The pressure vessel comprises a single and sealed volume that is confined between a bottom and a top plate. The pressure vessel has four circular windows that are laterally arranged, $90^{\circ}$ apart. The material can either be aluminum oxide or quartz. A water cooling is used to keep the top plate at a constant temperature. The bottom plate is heated by a builtin heating wire. The transparent top plate allows to visualize the cloud patterns in the horizontal $x-y$-plane. A light source is used to illuminate the convection cell from above. The solid red line marks the incoming light beam that passes through a 50/50 beam splitter. The reflected light from the bottom plate is captured by a digital 


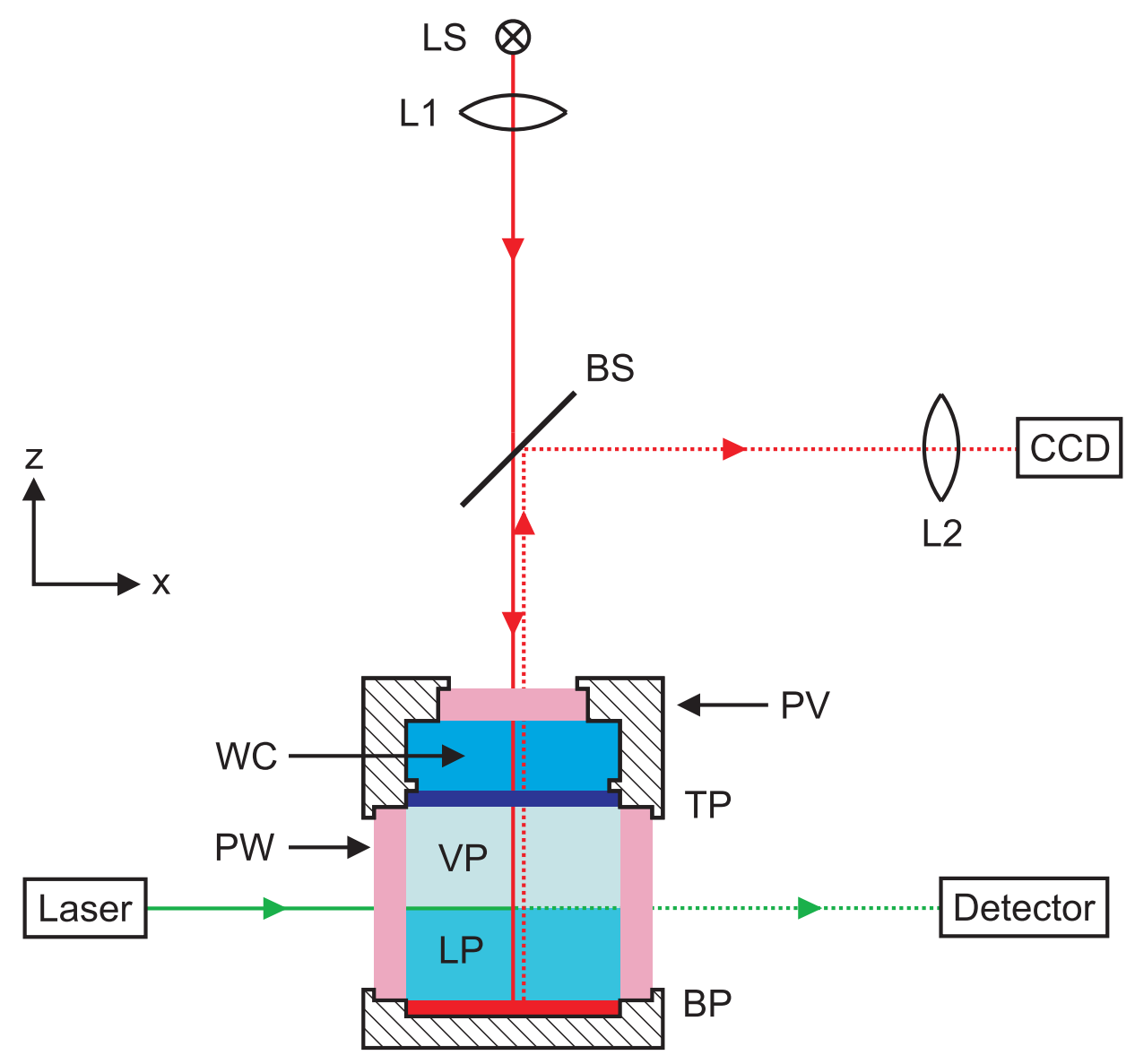

Figure 5.1: Schematic drawing of the experimental apparatus for the scattering experiments. $\mathrm{BP}=$ bottom plate, $\mathrm{BS}=$ beam splitter, $\mathrm{CCD}=$ digital camera, $\mathrm{L} 1=$ lens, $\mathrm{L} 2=$ lens, $\mathrm{LP}=$ liquid phase, $\mathrm{LS}=$ light source, $\mathrm{PV}=$ pressure vessel, $\mathrm{PW}=$ pressure window, $\mathrm{TP}=$ top plate, $\mathrm{VP}=$ vapor phase, $\mathrm{WC}=$ water cooling.

camera. The four lateral windows allow for a horizontal access to the convection cell. The method of dynamic light scattering (DLS) can be applied in order to investigate the region above the liquid-vapor interface where the cloud patterns are supposed to form. It is suggested to scan the gaseous phase with the laser beam in order to determine the extent of the cloud patterns in the $z$-direction. 


\section{Appendix A}

\section{A.1 Determination of the molar concentration of He in the gaseous phase}

Fig. 2.5 illustrates the molar concentration of $\mathrm{He}$ in the liquid and the gaseous phase of a mixture of $\mathrm{SF}_{6}$ and $\mathrm{He}$ for two different temperatures. The molar concentration of He in the gaseous phase is much larger compared to the molar concentration in the liquid phase. He tends to remain in the gaseous phase since the vapor pressure and also the solubility of $\mathrm{He}$ in the liquid phase of $\mathrm{SF}_{6}$ is very low. Thus the concentration of $\mathrm{He}$ in the liquid phase of $\mathrm{SF}_{6}$ is neglected. Furthermore the time constant for the mass diffusion of $\mathrm{He}$ into the liquid phase of $\mathrm{SF}_{6}$ is of the order of several days as shown in Section 4.1 .2 whereas the time interval for the measurements is usually less than $1 \mathrm{~h}$. Therefore the molar concentration of He in the gaseous phase is considered as being constant during the measurements. Since He is diffusing into the liquid phase of $\mathrm{SF}_{6}$, the pressure in the system is decreasing over time. During the measurements the pressure in the system is logged and by knowing the instantaneous pressure in the system, the actual molar concentration of He in the gaseous phase can be determined at each point in time.

In order to estimate the molar concentration of $\mathrm{He}$ in the gaseous phase, it is assumed 
that the gaseous phase is saturated with $\mathrm{SF}_{6}$ vapor. Since a liquid and a vapor phase of $\mathrm{SF}_{6}$ coexist, the saturation vapor pressure of $\mathrm{SF}_{6}$ adjusts according to the LVCL in Fig. 2.2. Furthermore ideal gas conditions are assumed for the $\mathrm{SF}_{6}$ and He mixture. For the subsequent calculations the two mixture components $\mathrm{SF}_{6}$ and He are denoted with the index 1 and 2 respectively. According to Dalton's law, the total pressure in the system equals the sum of the partial pressures of the two components

$$
p=p_{1}+p_{2}
$$

where $p$ is the total pressure in the system and $p_{1}$ and $p_{2}$ are the partial pressures of $\mathrm{SF}_{6}$ and He respectively [5]. The total pressure in the system is measured by employing the pressure gauge PG1 in Fig. 3.1. The saturation vapor pressure $p_{1}$ of $\mathrm{SF}_{6}$ is known from the LVCL in Fig. 2.2. The partial pressure of He is determined by applying Eq. A.1). In order to determine the molar concentration of $\mathrm{He}$ in the gaseous phase, the mole masses of the two components must be known. The total number of moles $n$ in the system equals the sum of the number of moles of the two components

$$
n=n_{1}+n_{2}
$$

where $n_{1}$ and $n_{2}$ denote the number of moles of $\mathrm{SF}_{6}$ and He respectively [5]. The number of moles of the two components $\mathrm{SF}_{6}$ and $\mathrm{He}$ can be written in terms of the density $\rho_{i},(i=1,2)$ and the molar mass $M_{i},(i=1,2)$ and it follows

$$
\begin{aligned}
& n_{1}=\frac{m_{1}}{M_{1}}=\frac{\rho_{1} V}{M_{1}}, \\
& n_{2}=\frac{m_{2}}{M_{2}}=\frac{\rho_{2} V}{M_{2}} .
\end{aligned}
$$


By employing the ideal gas law

$$
p V=n R T
$$

the following relations can be derived

$$
\begin{aligned}
& p_{1}=\frac{n_{1} R T}{V}, \\
& p_{2}=\frac{n_{2} R T}{V},
\end{aligned}
$$

where $R$ is the universal gas constant, $T$ the temperature and $V$ the volume of the system [5]. If $p_{1}$ and $p_{2}$ are known, Eq. (A.1) can be written in the form

$$
\begin{aligned}
p & =p_{1}+p_{2}=n_{1} \frac{R T}{V}+n_{2} \frac{R T}{V}=\left(n_{1}+n_{2}\right) \frac{R T}{V}, \\
& =\left(n_{1}+n_{2}\right) \frac{p}{n}=\frac{n_{1}}{n} p+\frac{n_{2}}{n} p .
\end{aligned}
$$

According to Fig. 2.5, the total molar concentration in the gaseous phase of $\mathrm{SF}_{6}$ and He is $y=y_{1}+y_{2}$. Hence for the two components in the gaseous phase it follows

$$
\begin{aligned}
& y_{1}=\frac{n_{1}}{n}=\frac{\rho_{1} M_{1}^{-1}}{\rho_{1} M_{1}^{-1}+\rho_{2} M_{2}^{-1}}, \\
& y_{2}=\frac{n_{2}}{n}=\frac{\rho_{2} M_{2}^{-1}}{\rho_{1} M_{1}^{-1}+\rho_{2} M_{2}^{-1}} .
\end{aligned}
$$




\section{A.2 Estimation of the Rayleigh number for the liquid phase of $\mathrm{SF}_{6}$}

By employing the thermophysical properties of liquid $\mathrm{SF}_{6}$, the Rayleigh number $R a$ and the critical temperature difference $\Delta T_{\mathrm{c}}$ for the onset of the thermal convection in the liquid phase of $\mathrm{SF}_{6}$ are estimated. The estimate for $R a$ allows to determine whether the heat transport in the liquid phase of $\mathrm{SF}_{6}$ is dominated by thermal diffusion or by thermal convection. The height of the liquid phase of $\mathrm{SF}_{6}$ in the convection cell is estimated to be $H_{\mathrm{lp}}=1 \times 10^{-2} \mathrm{~m}$. The critical Rayleigh number $R a_{\mathrm{c}}$ for rigid boundaries at the bottom and the top is given in Eq. (2.34). Since the thermal conductivity of the liquid phase of $\mathrm{SF}_{6}$ and the gaseous phase of $\mathrm{SF}_{6}$ and $\mathrm{He}$ are nearly equal, it is assumed that the temperature drop across the liquid and the gaseous phase is of the same order. Therefore the temperature drop across the liquid phase of $\mathrm{SF}_{6}$ is assumed to be $\Delta T_{\mathrm{L}}=\Delta T / 2$. The mean temperature $T=T_{\mathrm{b}}-\Delta T_{\mathrm{L}} / 2$ is used to determine the thermophysical properties of the liquid phase of $\mathrm{SF}_{6}$ in Table

\begin{tabular}{|c|c|c|}
\hline $\begin{array}{l}\text { Thermophysical properties } \\
\left(T=317.988 \mathrm{~K}, p=53.974 \mathrm{bar}, \Delta T_{\mathrm{L}}=0.226 \mathrm{~K}\right)\end{array}$ & Value & \\
\hline${ }^{\mathrm{a}}$ Thermal conductivity $\lambda(T, p)$ & 0.05084 & $\mathrm{~W} \mathrm{~m}^{-1} \mathrm{~K}^{-1}$ \\
\hline${ }^{\mathrm{a}}$ Density $\rho(T, p)$ & 1229.5 & $\mathrm{~kg} \mathrm{~m}^{-3}$ \\
\hline${ }^{a}$ Heat capacity $c_{p}(T, p)$ & 1371.3 & $\mathrm{~J} \mathrm{~kg}^{-1} \mathrm{~K}^{-1}$ \\
\hline a Kinematic viscosity $\eta(T, p)$ & \multicolumn{2}{|c|}{$7.7723 \times 10^{-8} \mathrm{~N} \mathrm{~s} \mathrm{~m}^{-2}$} \\
\hline${ }^{\mathrm{a}}$ Heat expansion coefficient $\alpha(T, p)$ & 0.01122 & $\mathrm{~K}^{-1}$ \\
\hline${ }^{\mathrm{a}}$ Dynamic viscosity $v(T, p)$ & 9.5558 & $\times 10^{-5} \mathrm{~m}^{2} \mathrm{~s}^{-1}$ \\
\hline${ }^{\mathrm{a}}$ Thermal diffusivity $\kappa(T, p)$ & 3.0156 & $\times 10^{-8} \mathrm{~m}^{2} \mathrm{~s}^{-1}$ \\
\hline
\end{tabular}

${ }^{a}$ Data taken from [52].

Table A.1: Thermophysical properties of liquid $\mathrm{SF}_{6}$. 
A.1. It is further assumed that the liquid phase of $\mathrm{SF}_{6}$ contains no He. By inserting the thermophysical properties in Table A.1 into Eq. (2.31), the Rayleigh number is determined to

$$
R a_{\mathrm{L}}=1.4 \times 10^{7}
$$

and the critical temperature difference is found to

$$
\begin{aligned}
\Delta T_{\mathrm{cL}} & =\frac{\kappa v R a_{\mathrm{c}}}{\alpha g H_{\mathrm{lp}}^{3}} \\
& =2.7 \times 10^{-5} \mathrm{~K} .
\end{aligned}
$$

The result in Eq. A.9) shows that $\Delta T_{\mathrm{cL}}$ is about four orders of magnitude smaller than the temperature difference $\Delta T_{\mathrm{L}}$. Therefore it is likely that the convection in the liquid phase of $\mathrm{SF}_{6}$ is highly turbulent.

\section{A.3 Estimation of the Rayleigh number for the gaseous phase of $\mathrm{SF}_{6}$ and $\mathrm{He}$}

The observed cloud patterns in Section 4.3.1 indicate that the flow in the gaseous phase of $\mathrm{SF}_{6}$ and He is likely to be turbulent. The partial pressure and the molar concentration of $\mathrm{He}$ in the gaseous phase are determined by applying the formulas in Eqs. (A.1) and (A.7b). The temperature drop across the gaseous phase of $\mathrm{SF}_{6}$ and He is assumed to be $\Delta T_{\mathrm{V}}=\Delta T / 2$. The mean temperature $T=T_{\mathrm{t}}+\Delta T_{\mathrm{V}} / 2$ is used to determine the thermophysical properties of the gaseous phase of $\mathrm{SF}_{6}$ and $\mathrm{He}$ in Table A.2. Since the instantaneous pressure in the system is logged, the actual molar concentration of $\mathrm{He}$ in the gaseous phase of $\mathrm{SF}_{6}$ and $\mathrm{He}$ can be determined at each 


\begin{tabular}{|c|c|c|}
\hline $\begin{array}{l}\text { Thermophysical properties } \\
\left(\begin{array}{l}T=317.763 \mathrm{~K}, p=53.974 \text { bar, } y_{2}=14.5 \% \\
\left.\Delta T_{\mathrm{V}}=0.226 \mathrm{~K}\right)\end{array}\right.\end{array}$ & Value & \\
\hline${ }^{\mathrm{a}}$ Thermal conductivity $\lambda\left(T, p, y_{2}\right)$ & 0.04975 & $\mathrm{~W} \mathrm{~m} \mathrm{~m}^{-1} \mathrm{~K}^{-1}$ \\
\hline${ }^{a}$ Density $\rho\left(T, p, y_{2}\right)$ & 455.4 & $\mathrm{~kg} \mathrm{~m}^{-3}$ \\
\hline${ }^{\text {a }}$ Heat capacity $c_{p}\left(T, p, y_{2}\right)$ & 786.04 & $\mathrm{~J} \mathrm{~kg}^{-1} \mathrm{~K}^{-1}$ \\
\hline${ }^{\text {a }}$ Kinematic viscosity $\eta\left(T, p, y_{2}\right)$ & 2.606 & $\times 10^{-5} \mathrm{~N} \mathrm{~s} \mathrm{~m}^{-2}$ \\
\hline${ }^{\mathrm{a}}$ Heat expansion coefficient $\alpha\left(T, p, y_{2}\right)$ & 0.01696 & $\mathrm{~K}^{-1}$ \\
\hline Dynamic viscosity $v\left(T, p, y_{2}\right)$ & 5.7224 & $\times 10^{-8} \mathrm{~m}^{2} \mathrm{~s}^{-1}$ \\
\hline Thermal diffusivity $\kappa\left(T, p, y_{2}\right)$ & 1.3898 & $\times 10^{-7} \mathrm{~m}^{2} \mathrm{~s}^{-1}$ \\
\hline
\end{tabular}

a Data taken from [1].

Table A.2: Thermophysical properties of the gaseous phase of $\mathrm{SF}_{6}$ and He.

point in time. The molar concentration of $\mathrm{He}$ is determined to $y_{2}=14.5 \%$. The height of the gaseous phase of $\mathrm{SF}_{6}$ and $\mathrm{He}$ in the convection cell is estimated to be $H_{\mathrm{gp}}=1 \times 10^{-2} \mathrm{~m}$. By inserting the thermophysical properties in Table A.2 into Eq. (2.31), the Rayleigh number is determined to

$$
R a_{\mathrm{V}}=6.4 \times 10^{6}
$$

and the critical temperature difference is found to

$$
\begin{aligned}
\Delta T_{\mathrm{cV}} & =\frac{\kappa v R a_{\mathrm{c}}}{\alpha g H_{\mathrm{gp}}^{3}} \\
& =6.0 \times 10^{-5} \mathrm{~K} .
\end{aligned}
$$

The result in Eq. A.11) shows that $\Delta T_{\mathrm{cV}}$ is about four orders of magnitude smaller than the temperature difference $\Delta T_{\mathrm{V}}$. Since $R a \gg R a_{\mathrm{c}}$, the convection in the gaseous 
phase of $\mathrm{SF}_{6}$ and $\mathrm{He}$ is expected to be highly turbulent. Therefore it is very likely that the turbulent flow in the gaseous phase of $\mathrm{SF}_{6}$ and $\mathrm{He}$ is causing the dynamics of the cloud patterns in Section 4.3.1.

\section{A.4 Turbulent plume velocity and turbulent mixing time}

The buoyant velocity $U_{\mathrm{b}}$ of a turbulent plume is estimated for the gaseous phase of $\mathrm{SF}_{6}$ and He by applying the formulas given in [45]. The turbulent plume or buoyant velocity is the maximum velocity that an uprising plume can achieve. The velocity of the cold downward falling plumes is expected to be of the same order of magnitude as the velocity of the uprising plumes. By employing the thermophysical properties given in Table A.3, the buoyancy flux $B$ is determined to

$$
\begin{aligned}
B & =\frac{\alpha g P}{\rho c_{\mathrm{p}}} \\
& =6.6 \times 10^{-8} \mathrm{~m}^{2} \mathrm{~s}^{-3},
\end{aligned}
$$

where $\alpha$ is the thermal expansion coefficient, $g$ the gravitational constant, $\rho$ the density and $c_{\mathrm{p}}$ the heat capacity of the gaseous phase of $\mathrm{SF}_{6}$ and He. By knowing the buoyancy flux, the buoyant velocity $U_{\mathrm{b}}$ is calculated to

$$
\begin{aligned}
U_{\mathrm{b}} & =\left(\frac{B}{H_{\mathrm{gp}}}\right)^{\frac{1}{3}} \\
& =0.0181 \mathrm{~m} \mathrm{~s}^{-1} \approx 18 \mathrm{~mm} \mathrm{~s}^{-1}
\end{aligned}
$$




\begin{tabular}{|c|c|c|}
\hline $\begin{array}{l}\text { Thermophysical properties } \\
\left(T=317.763 \mathrm{~K}, p=53.974 \mathrm{bar}, y_{2}=14.5 \%\right)\end{array}$ & Value & \\
\hline${ }^{\mathrm{a}}$ Density $\rho\left(T, p, y_{2}\right)$ & 455.4 & $\mathrm{~kg} \mathrm{~m}^{-3}$ \\
\hline${ }^{\mathrm{a}}$ Heat capacity $c_{\mathrm{p}}\left(T, p, y_{2}\right)$ & 786.04 & $\mathrm{~J} \mathrm{~kg}^{-1} \mathrm{~K}^{-1}$ \\
\hline${ }^{\mathrm{a}}$ Heat expansion coefficient $\alpha\left(T, p, y_{2}\right)$ & \multicolumn{2}{|c|}{$0.01696 \mathrm{~K}^{-1}$} \\
\hline Heating power $P$ & 0.1413 & $\mathrm{~W}$ \\
\hline
\end{tabular}

a Data taken from [1].

Table A.3: Thermophysical properties of the gaseous phase of $\mathrm{SF}_{6}$ and He.

In order to estimate how long the mixing process in the gaseous phase of $\mathrm{SF}_{6}$ and $\mathrm{He}$ takes if a new amount of $\mathrm{He}$ is filled into the convection cell, the turbulent mixing time $t_{\mathrm{mt}}$ is determined as defined in [45]. It is assumed that both gases $\mathrm{SF}_{6}$ and $\mathrm{He}$ are mixed due to the turbulent flow in the gaseous phase. In order to calculate the mixing time, the turbulent diffusion coefficient $D_{\mathrm{td}}$ is determined to

$$
\begin{aligned}
D_{\mathrm{td}} & =U_{\mathrm{b}} H_{\mathrm{gp}} \\
& =2.0 \times 10^{-4} \mathrm{~m}^{2} \mathrm{~s}^{-1},
\end{aligned}
$$

where $H_{\mathrm{gp}}$ is the height of the gaseous phase of $\mathrm{SF}_{6}$ and $\mathrm{He}$ in the convection cell. The turbulent mixing time $t_{\mathrm{mt}}$ is defined as follows

$$
t_{\mathrm{mt}}=\frac{z^{2}}{D_{\mathrm{td}}} .
$$


By knowing $D_{\mathrm{td}}$, the vertical and the horizontal mixing time denoted with $t_{\mathrm{vmt}}$ and $t_{\mathrm{hmt}}$ respectively is determined to

$$
\begin{gathered}
\text { vertical mixing time: } t_{\mathrm{vmt}}\left(z=H_{\mathrm{gp}}\right) \approx 1 \mathrm{~s}, \\
\text { horizontal mixing time: } t_{\mathrm{hmt}}(z=W) \approx 21 \mathrm{~s},
\end{gathered}
$$

where $W$ is the width of the convection cell. $t_{\mathrm{vmt}}$ and $t_{\mathrm{hmt}}$ are both of the order of seconds. The result in Eqs. (A.16a) and (A.16b) shows that the mixing time in the gaseous phase of $\mathrm{SF}_{6}$ and $\mathrm{He}$ is less than $1 \mathrm{~min}$. 


\section{Acknowledgements}

In the end I like to thank the following people for supporting me with their knowledge and expertise during the time as a doctoral student. First I want to thank Udo Schminke and the team from the mechanical workshop for constructing, building and modifying parts and components for the experimental apparatus. They always bore a helping hand in solving mechanical problems of any kind. Next I like to thank the technical stuff of our department, Andreas Kopp, Andreas Renner and Dr. Arthur Kubitzek for supporting me with their advice and for providing me with special solutions whenever problems arose. I also like to thank Dr. Stephan Weiss for his advice and for supplying me with technical details about the experimental apparatus. Also many thanks to Dr. Matthias Schröter and Prof. Jürgen Vollmer for very helpful and useful suggestions they made concerning our project. Next I like to thank Dr. Gregory Bewley and Dr. Haitao Xu for supporting me with their knowledge and expertise. In particular I like to thank Dr. Alexei Krekhov for many informative and enlightening discussions I had with him. I also like to thank our research collaborators especially Prof. Günther Ahlers for making some useful and helpful contributions for our project. Finally I like to thank the members of my thesis committee, Prof. Bjoern Hof, Dr. Holger Nobach and Prof. Andreas Tilgner for supporting and supervising me during the time as a doctoral student. Last but not least I like to thank the head of our department Prof. Eberhard Bodenschatz for supporting me with his knowledge and expertise. He was the one who had the idea for the whole project. Last I like to thank my parents and all members of our department and all those I forgot to mention. 


\section{Bibliography}

[1] G. Ahlers et al. Software for the calculation of fluid properties relevant to convection. Department of Physics, University of California, Santa Barbara, CA 93106, 2012.

[2] M. P. Anisimov. Nucleation: theory and experiment. Russ. Chem. Rev., 72(7):591, 2003.

[3] M. P. Anisimov, E. G. Fominykh, S. V. Akimov, and P. K. Hopke. Vaporgas/liquid nucleation experiments: A review of the challenges. J. Aerosol Sci., 40(9):733-746, 2009.

[4] G. B. Arfken and H. J. Weber. Mathematical Methods for Physicists. Elsevier Academic Press, Amsterdam, 6th edition, 2005.

[5] P. Atkins and J. de Paula. Physical Chemistry. Oxford University Press, Oxford, 8th edition, 2006.

[6] A. Belmonte, A. Tilgner, and A. Libchaber. Temperature and velocity boundary layers in turbulent convection. Phys. Rev. E, 50(1):269-279, 1994.

[7] E. P. Bernard and W. Krauth. Two-Step Melting in Two Dimensions: First-Order Liquid-Hexatic Transition. Phys. Rev. Lett., 107(15):155704, 2011.

[8] M. Bestehorn and D. Merkt. Regular Surface Patterns on Rayleigh-Taylor Unstable Evaporating Films Heated from Below. Phys. Rev. Lett., 97(12):127802, 2006.

[9] D. Blair and E. Dufresne. Matlab Particle Tracking Code. http://physics. georgetown. edu/matlab/index.html, 2013. (Matlab adaptation of the IDL Particle Tracking software developed by Grier, D. and Crocker, J. and Weeks, E.).

[10] J. M. Burgess, A. Juel, W. D. McCormick, J. B. Swift, and H. L. Swinney. Suppression of Dripping from a Ceiling. Phys. Rev. Lett., 86(7):1203-1206, 2001.

[11] J. Burton, A. T. Nguyen Le, J. E. Rutledge, and P. Taborek. Supercooling Helium Vapor: Nucleation and Fog Formation Induced by Strong Evaporation. J. Low Temp. Phys., 134(1-2):275-280, 2004. 
[12] F. H. Busse. Nonlinear properties of thermal-convection. Rep. Prog. Phys., 41(12):1929, 1978.

[13] R. F. Cahalan and J. H. Joseph. Fractal Statistics of Cloud Fields. Mon. Weather Rev., 117:261-272, 1989.

[14] T. F. Chan and L. A. Vese. Active Contours Without Edges. IEEE Trans. Image Processing, 10:266-277, 2001.

[15] S. Chandrasekhar. Hydrodynamic and Hydromagnetic Stability. Oxford University Press, London, 1961.

[16] D. C. Cho and R. P. Stein. Steam condensation on the underside of a horizontal surface. In Proceedings of Third International Topical Meeting on Nuclear Power Plant Thermal Hydr. and Operations, 1988.

[17] B. Chu. Critical Opalescence. Berich. Bunsen. Gesell., 76(3-4):202-215, 1972.

[18] J. R. de Bruyn, E. Bodenschatz, S. W. Morris, S. P. Trainoff, Y. Hu, D. S. Cannell, and G. Ahlers. Apparatus for the study of Rayleigh-Bénard convection in gases under pressure. Rev. Sci. Instrum., 67:2043-2067, 1996.

[19] J. de Swaan Arons and G. A. M. Diepen. Gas-Gas Equilibria. J. Chem. Phys., 44:2322-2330, 1966.

[20] Y.-B. Du and P. Tong. Turbulent thermal convection in a cell with ordered rough boundaries. J. Fluid Mech., 407:57-84, 2000.

[21] F. Ehrler and K. Schaber. J5 Spontane Kondensation und Aerosolbildung. In VDI-Wärmeatlas, VDI-Buch, pages 1053-1082. Springer-Verlag, Berlin, 2013.

[22] K. A. Emanuel. ATMOSPHERIC CONVECTION. Oxford University Press, New York, 1994.

[23] M. Fermigier, L. Limat, J. E. Wesfreid, P. Boudinet, and C. Quilliet. Twodimensional patterns in Rayleigh-Taylor instability of a thin layer. J. Fluid Mech., 236:349-383, 1992.

[24] M. T. Gastner, B. Oborny, D. K. Zimmermann, and G. Pruessner. Transition from Connected to Fragmented Vegetation across an Environmental Gradient: Scaling Laws in Ecotone Geometry. Am. Nat., 174(1):E23-E39, 2009.

[25] A. V. Getling. Rayleigh-Bénard Convection - Structures and Dynamics. World Scientific Publishing Co. Pte. Ltd., Singapore, 1998.

[26] R. C. Gonzalez, R. E. Woods, and S. L. Eddins, editors. Digital Image Processing Using MATLAB. Pearson Education Inc., Upper Saddle River, NJ, 2004. 
[27] A. Gripshoefer and S. Westerheide. Matlab toolbox for pet/ct image segmentation with the Chan-Vese model. http://wwwmath.uni-muenster.de/num/ Vorlesungen/MedizinUndMathematik/PETCT, 2008.

[28] W. M. Haynes. „Critical Constants of Inorganic Compounds”. In W. M. Haynes, editor, CRC Handbook of Chemistry and Physics. CRC Press/Taylor and Francis, Boca Raton, FL, 94th edition, 2014.

[29] R. H. Heist, J. Ahmed, and M. Janjua. Effects of Background Gases on the Homogeneous Nucleation of Vapors. 2. J. Phys. Chem., 99(1):375-383, 1995.

[30] R. H. Heist and H. He. Review of Vapor to Liquid Homogeneous Nucleation Experiments from 1968 to 1992. J. Phys. Chem. Ref. Data, 23(5):781, 1994.

[31] R. H. Heist, M. Janjua, and J. Ahmed. Effects of Background Gases on the Homogeneous Nucleation of Vapors. 1. J. Phys. Chem., 98(16):4443-4453, 1994.

[32] A. W. Hodgson. Homogeneous nucleation. Adv. Colloid Interface Sci., 21(34):303-327, 1984.

[33] C. E. Holloway and J. D. Neelin. Moisture Vertical Structure, Column Water Vapor, and Tropical Deep Convection. J. Atmos. Sci., 66(6):1665-1683, 2009.

[34] I. K. Huhtiniemi and M. L. Corradini. Condensation in the presence of noncondensable gases. Nucl. Eng. Des., 141(3):429-446, 1993.

[35] V. I. Kalikmanov. Nucleation Theory, volume 860 of Lecture Notes in Physics. Springer Science+Business Media, Dordrecht, 2013.

[36] J. L. Katz. Condensation of a Supersaturated Vapor. I. The Homogeneous Nucleation of the n-Alkanes. J. Chem. Phys., 52(9):4733, 1970.

[37] J. L. Katz and B. J. Ostermier. Diffusion Cloud-Chamber Investigation of Homogeneous Nucleation. J. Chem. Phys., 47(2):478-487, 1967.

[38] S. Kotake and I. I. Glass. Flows with nucleation and condensation. Prog. Aerosp. Sci, 19:129-196, 1979.

[39] P. K. Kundu and I. M. Cohen. Fluid Mechanics. Academic press, San Diego, CA, 2nd edition, 2002.

[40] A. Laaksonen, V. Talanquer, and D. W. Oxtoby. Nucleation: Measurements, Theory, and Atmospheric Applications. Annu. Rev. Phys. Chem., 46(1):489-524, 1995.

[41] D. Lamb and J. Verlinde. Physics and Chemistry of Clouds. Cambridge University Press, Cambridge, 2011. 
[42] L. D. Landau and E. M. Lifshitz. Fluid Mechanics. (volume 6 of Course of theoretical physics). Pergamon Press, Oxford, 2nd edition, 1987.

[43] M. Lappa. Thermal Convection: Patterns, Evolution and Stability. John Wiley \& Sons Ltd., United Kingdom, 2010.

[44] D. J. Lewis. The Instability of Liquid Surfaces when Accelerated in a Direction Perpendicular to their Planes. II. P. Roy. Soc. Lond A, 202:81-96, 1950.

[45] E. J. List. Turbulent Jets and Plumes. Annu. Rev. Fluid Mech., 14(1):189-212, 1982.

[46] S. Lovejoy. Area-Perimeter Relation for Rain and Cloud Areas. Science, 216:185$187,1982$.

[47] S. P. Malinowski and I. Zawadzki. On the Surface of Clouds. J. Atmos. Sci., 50(1):5-13, 1993.

[48] P. Manneville. Instabilities, Chaos and Turbulence. World Scientific Publishing Co. Pte. Ltd., Singapore, 2010.

[49] F. Marsik, T. Nemec, J. Hruby, P. Demo, Z. Kozisek, V. Petr, and M. Kolovratnik. Binary Homogeneous Nucleation in Selected Aqueous Vapor Mixtures. J. Solution Chem., 37(12):1671-1708, 2008.

[50] D. Mumford and J. Shah. Optimal Approximations by Piecewise Smooth Functions and Associated Variational Problems. Commun. Pur. Appl. Math., 42:577$685,1989$.

[51] A. Natan. Fast 2D peak finder. http://www.mathworks.cn/matlabcentral/ fileexchange/37388-fast-2d-peak-finder, 2013.

[52] NIST. Reference Fluid Thermodynamic and Transport Properties, NIST Standard Reference Database 23, Version 9.1. Applied Chemicals and Material Division, National Institute of Standards and Technology, Boulder, CO 80305, 2013.

[53] M. J. de Oliveira. Equilibrium Thermodynamics. Springer-Verlag, Berlin, 2013.

[54] A. Oron, S. H. Davis, and S. G. Bankoff. Long-scale evolution of thin liquid films. Rev. Mod. Phys., 69(3):931-980, 1997.

[55] S. Osher and J. A. Sethian. Fronts Propagating with Curvature-Dependent Speed: Algorithms Based on Hamilton-Jacobi Formulations. J. Comput. Phys., 79:12-49, 1988.

[56] B. B. Plapp. SPIRAL PATTERN FORMATION IN RAYLEIGH-BÉNARD CONVECTION. PhD thesis, Cornell University, 1997. 
[57] B. E. Poling, J. M. Prausnitz, and J. P. O'Connell. The Properties of Gases and Liquids. McGraw-Hill, New York, 5th edition, 2001.

[58] J. M. Prausnitz, R. N. Lichtenthaler, and E. G. de Azevedo. Molecular Thermodynamics of Fluid-Phase Equilibria. Prentice Hall PTR, New Jersey, 3rd edition, 1999.

[59] H. R. Pruppacher and J. D. Klett. Microphysics of Clouds and Precipitation, volume 18 of Atmospheric and oceanographic sciences library. Springer Science+Business Media B.V., Dordrecht, 2nd revised edition, 2010.

[60] S. Rasenat, G. Hartung, B. L. Winkler, and I. Rehberg. The shadowgraph method in convection experiments. Exp. Fluid., 7(6):412-420, 1989.

[61] L. Rayleigh. The Form of Standing Waves on the Surface of Running Water. Proc. Lond. Math. Soc., s1-15(1):69-78, 1883.

[62] F. S. Rys and A. Waldvogel. Fractal shape of hail clouds. Phys. Rev. Lett., 56(7):784-787, 1986.

[63] N. Schaeffer, F. Utheza, F. Garnier, and G. Lauriat. Stable stratification alteration in a thermal diffusion cloud chamber. J. Chem. Phys., 113(18):8085-8092, 2000.

[64] A. P. Siebesma and H. J. J. Jonker. Anomalous Scaling of Cumulus Cloud Boundaries. Phys. Rev. Lett., 85(1):214-217, 2000.

[65] P. J. Steinhardt, D. R. Nelson, and M. Ronchetti. Bond-orientational order in liquids and glasses. Phys. Rev. B, 28(2):784-805, 1983.

[66] G. Taylor. LAMINAR FILM CONDENSATION ON THE UNDERSIDE OF HORIZONTAL AND INCLINED SURFACES. P. Roy. Soc. Lond. A, 201:192-196, 1967.

[67] M. v. Smoluchowski. Molekular-kinetische Theorie der Opaleszenz von Gasen im kritischen Zustande, sowie einiger verwandter Erscheinungen. Ann. Phys., 330(2):205-226, 1908.

[68] H. Vehkamäki. Classical Nucleation Theory in Multicomponent Systems. SpringerVerlag, Berlin, 2006.

[69] P. E. Wagner and R. Strey. Measurements of homogeneous nucleation rates for n-nonane vapor using a two-piston expansion chamber. J. Chem. Phys., 80(10):5266-5275, 1984.

[70] P. P. Wegener and P. Mirabel. Homogeneous nucleation in supersaturated vapors. Naturwissenschaften, 74(3):111-119, 1987. 
[71] P. P. Wegener and A. A. Pouring. Experiments on Condensation of Water Vapor by Homogeneous Nucleation in Nozzles. Phys. Fluids, 7(3):352, 1964.

[72] P. P. Wegener and B. J. C. Wu. Gasdynamics and homogeneous nucleation. Adv. Colloid Interface Sci., 7(1):325-417, 1977.

[73] T. Weidauer, O. Pauluis, and J. Schumacher. Cloud patterns and mixing properties in shallow moist Rayleigh-Bénard convection. New J. Phys., 12:105002, 2010.

[74] J. Winkelmann. Diffusion of helium (1); water (2). In M. D. Lechner, editor, Gases in Gases, Liquids and their Mixtures, number 15A in Landolt-Börnstein - Group IV Physical Chemistry, pages 2029-2031. Springer Berlin Heidelberg, 2007.

[75] P. Ye, A. Bertelsmann, and R. H. Heist. Nucleation of sulfur hexafluoride. In AIP Conference Proceedings, volume 534, pages 19-22. AIP Publishing, 2000. 